\title{
The structure and degree of dependence in government bond markets
}

\author{
Nebojsa Dimic $^{\text {a }}$, Vanja Piljak ${ }^{\text {a, }}$, Laurens Swinkels ${ }^{\text {b,c }}$, Milos Vulanovic ${ }^{\mathrm{d}}$ \\ ${ }^{\text {a }}$ University of Vaasa, School of Accounting and Finance, Vaasa, Finland \\ ${ }^{\mathrm{b}}$ Erasmus University, School of Economics, Rotterdam, the Netherlands \\ ${ }^{\mathrm{c}}$ Robeco Institutional Asset Management, Rotterdam, the Netherlands \\ ${ }^{\mathrm{d}}$ EDHEC Business School, Lille, France
}

\section{A R T I C L E I N F O}

\section{JEL classification:}

G01

G15

Keywords:

Dependence structure

Emerging and frontier markets

Quantile regression

Sovereign bonds

Financial crisis

\begin{abstract}
A B S T R A C T
Our study provides new evidence on asymmetric dependencies in international government bond markets, by examining bonds from developed, emerging, and frontier countries, using a quantile regression methodology. We find that the dependence structure for emerging and frontier markets significantly changes during financial crisis periods, which we show has important implications for international diversification of investment strategies. Moreover, we also examine in detail stock-bond correlations and uncover several instances of decoupling. In contrast, developed markets exhibit a more stable dependence pattern. In addition, we document that the degree and structure of dependence vary when foreign currencies are hedged or unhedged, and across bond maturity segments.
\end{abstract}

\section{Introduction}

We examine the degree and structure of dependence in international government bond markets. Measuring dependence is of high relevance for asset allocation and risk management, especially since the structure of the dependence helps to assess potential diversification benefits during both tranquil and crisis periods; see Baur (2013). Correct estimation of dependence is particularly essential for a proper understanding of the functioning and performance of government bond markets, which are often assumed to be risk-free in nominal terms for local investors. International investors, however, are facing the risk of a sovereign (selectively) defaulting on its debt or exchange rate risk involved with holding foreign assets (Arellano and Ramanarayanan, 2012; Bhatta et al., 2017; Lustig and Verdelhan, 2019). Understanding better how these financial market risks vary in periods with and without stress is of crucial importance to investors constructing a diversified government bond portfolio.

The literature on the dependencies across financial markets is extensive, with a majority of studies concentrating on the modeling of dependence and utilizing: correlations, extreme value techniques, or copula-based frameworks. ${ }^{1}$ The copula models have been used to address asymmetric dependence structures between financial variables, such as: dependence across international equity markets

\footnotetext{
* Corresponding author.

E-mail addresses: nebojsa.dimic@uva.fi (N. Dimic), vanja.piljak@uva.fi (V. Piljak), 1swinkels@ese.eur.nl (L. Swinkels), milos.vulanovic@edhec. edu (M. Vulanovic).

${ }^{1}$ For approaches based on correlation and extreme value techniques, see Longin and Solnik (2001), Ang and Chen (2002), Beine et al. (2010), and Bhatti and Nguyen (2012). For a detailed discussion on modeling dependence, see Embrechts et al. (2002) and Garcia and Tsafack (2011). See also Patton (2012) for a comprehensive overview of the literature on copula-based models for economic and financial time series.
} 
(Hu, 2006; Rodriguez, 2007; Okimoto, 2008; Chollete et al., 2009; Markwat, 2014; Okimoto, 2014); exchange rates dependence (Patton, 2006); equity and foreign exchange market dependence (Ning, 2010); and equity and bond market dependence (Garcia and Tsafack, 2011).

$\mathrm{Hu}$ (2006) uses a copula framework to define the terms the degree and the structure of dependence. In Hu's (2006) settings, the application of the copula framework assumes an ad-hoc selection of an appropriate dependence function that captures the dependence structure between the variables of interest. Baur (2013) goes a step further and proposes an alternative approach for decomposing dependence into its degree and structure using quantile regressions estimation pioneered by Koenker and Bassett (1978). The advantage of this approach is that it provides a detailed picture of dependence, including asymmetric and non-linear relationships. Consequently, Baur (2013) defines the degree of dependence as the average over 99 quantile regression coefficients, and the structure of dependence as the pattern of the estimated coefficients across all 99 quantiles. His novel approach is advantageous in several ways. First, it eliminates the need for an ad hoc selection of a specific structure of the dependence. Second, it enables conditional dependence to be estimated directly. Finally, the estimation procedure provides the statistical significance of the dependence and the conditioning variables for each quantile of the distribution. Furthermore, his approach is also able to model dynamics in dependencies. Thus it is very suitable to examine how degree and structure of dependence are behaving during periods of turmoil in financial markets.

Our study is motivated by previous findings in the literature stating that asset returns exhibit higher correlation (dependence) during market downturns than market upturns (see Longin and Solnik, 2001; Ang and Bekaert, 2002). As a consequence, average dependence is not adequate to describe the dependence structure in financial markets. Particularly the proper estimation of dependence should include its upper and lower tail. In practice, the existence of asymmetric dependence has important implications for portfolio management in assessing the risk and diversification benefits of asset correlations (see Patton, 2004; Okimoto, 2008; Alcock and Satchell, 2018). While Baur (2013) utilizes the quantile-based empirical framework to test dependence structure in global equity markets, commodities, and exchange rates, he does not examine dependence structures in government bond markets. We fill that gap in the literature with this study. To be more precise, we address four research questions. First, we examine the degree and structure of dependence for three categories of government bond markets (developed, emerging, and frontier) relative to global bond market. We specifically focus on whether the dependence structure is asymmetric in the sense of exhibiting different upper and lower tail dependence. Furthermore, we analyze the role of bond maturity by examining differences in the degree and structure of dependence for short-, medium-, and long-term bonds. ${ }^{2}$ Second, we investigate whether the dependence structure is affected by the $2007-2008$ global financial crisis and the Eurozone debt crisis. Third, we examine the currency implications in the analysis of dependence structure using: (i) local currency returns converted to US dollar, (ii) returns hedged to US dollar using currency derivatives, and (iii) returns on US dollar-denominated government bonds. Fourth, we analyze the existence of flights-to-quality and contagion from global stock market to developed, emerging, and frontier bond markets.

This study contributes to the literature in four aspects. First, we provide a comprehensive analysis of the degree and structure of dependence in international government bond markets. In particular, we segment government bond markets into three categories: developed, emerging, and frontier, and analyze their dependence on the global bond market. We pay special attention to differences in dependence patterns across three categories of these bond markets. The distinction between the three aforementioned categories of bond markets is essential for proper international asset allocation management. Building on a common perception of emerging market bonds as "equity-like assets" and frontier markets bonds as "the next generation of emerging bond markets issuers" (Piljak and Swinkels, 2017a), our study provides useful insights for international portfolio managers, especially applicable in periods labeled as crisis times. Furthermore, we utilize the quantile-based approach proposed by Baur (2013) to decompose the dependence into its degree and structure. Finally, we investigate contagion in the degree and the structure of dependence during the global financial crisis and the Eurozone debt crisis, re-examining findings in Hu (2006) that financial turmoil can affect changes in both the degree and structure of dependence. To that end, we improve understanding of the contagion phenomenon by analyzing whether the contagion is more dominant for extreme returns (tails of the distribution) than for average returns.

Second, in addition to examining dependence for three categories of bond markets on the aggregate level, we also provide analysis on the regional and country level for emerging and frontier markets. The added value of regional and country perspectives is related to the fact that both emerging and frontier bond markets are heterogeneous groups with compelling differences in terms of sovereign credit ratings and levels of market integration (Christopher et al., 2012; Piljak, 2013; Šimović et al., 2016; Agur et al., 2019; Chaieb et al., 2020). Hence, we contribute to the growing body of literature on bond market integration in the domain of emerging and frontier markets.

Third, we examine the currency aspect of dependence by comparing returns hedged to the US dollar using currency derivatives with non-hedged returns. This insight is essential as the currency component of investing in foreign bonds can be the dominant driver of returns (Adler and Dumas, 1980; Burger and Warnock, 2007; Piljak and Swinkels, 2017b; Burger et al., 2018; Amstad et al., 2020). The currency aspect of dependence is essential for our study as we observe three categories of markets having different denomination practices. For developed markets, we use local-currency government bond markets, which are by far the largest debt markets for this group of countries and characterized by an investment-grade credit rating and free movement of capital. In the analysis, we examine returns converted and hedged to a common currency, namely the US dollar. For frontier markets, government bonds available to international investors are most frequently denominated in US dollars (Jeanneret and Souissi, 2016; Ottonello and Perez, 2019). The denomination of government bonds in US dollars reduces incentives of (the central banks of) these governments to revert to

\footnotetext{
${ }^{2}$ See Arellano and Ramanarayanan (2012) and Broner et al. (2013) for a comprehensive discussion on the importance of maturity structure in the context of emerging bond markets and crises periods.
} 
uncontrolled increases in money supply to fund repayment of the existing government debts (Farhi and Maggiori, 2018; Gennaioli et al., 2018). Simultaneously, bond denomination in US dollars protects foreign investors from domestic currency devaluation and enables the preservation of their purchasing power (Eichengreen et al., 2003). Emerging markets issue government bonds denominated both in local currency or US dollars (see, Cayon et al., 2018; Gilchrist et al., 2019), and we include each of these bond denominations in our analysis.

Finally, we contribute to the literature on stock-bond correlations and flight-to-quality, especially in the part of the literature devoted to emerging markets. Previous studies on stock-bond correlations in emerging markets concentrated on co-movement between stocks and bonds within individual emerging countries and analyzing factors that affect co-movement (Li and Zou, 2008, Panchenko and Wu, 2009; Christopher et al., 2012; Bianconi et al., 2013; Dimic et al., 2016). We extend this line of research by focusing on the co-movement between global stock market and three different categories of bond markets (emerging, frontier, and developed) while incorporating currency and hedging aspects, and testing for flight-to-quality and contagion during the global financial crisis and the Eurozone debt crisis.

The remainder of the paper is organized as follows. Section 2 provides a literature review, Section 3 describes our data, and Section 4 presents the methodological framework. In Section 5, we discuss our empirical results. Finally, Section 6 concludes.

\section{Literature review}

Our paper focuses on financial market dependencies. Still, it is closely related to the strands of literature concentrating on financial contagion, integration and co-movement dynamics in government bond markets, and stock-bond correlation with consequent flightto-quality. Thus, we provide here a brief description of related literature.

\subsection{Financial contagion}

One of the central issues in the financial contagion literature is examining changes in dependencies during the financial crisis period compared to tranquil periods. Different methodological approaches have been used to address this issue. In particular, a straightforward method to study contagion in equity markets is based on linear dependence measure; see Forbes and Rigobon (2002). More advanced techniques include co-exceedance measures in multinomial logistic regressions (e.g., Bae et al., 2003; Markwat et al., 2009) or in quantile regressions (Baur and Schulze, 2005); Markov switching models (Ang and Bekaert, 2002); switching-parameter copulas (Rodriguez, 2007), quantile-based approach (Baur, 2013; Cappiello et al., 2014).

In the vein of contagion in bond markets, Dungey et al. (2006) examine contagion in international bond markets during the Russian crisis in 1997, showing that both emerging and developed markets caught contagion during the crisis period. More recent studies concentrate on spillovers and contagion in Eurozone during the European sovereign debt crisis (Claeys and Vašícek, 2014; Gómez-Puig and Sosvilla-Rivero, 2014; Samarakoon, 2017; Bekiros et al., 2018; Caporin et al., 2018). In particular, Gómez-Puig and Sosvilla-Rivero (2014) provide evidence of contagion in the aftermath of the Eurozone sovereign debt crisis, while Claeys and Vašícek (2014) and Caporin et al. (2018) conclude that contagion has remained subdued. Samarakoon (2017) reports negative contagion stemming from the crisis countries to other stock markets. He also establishes that both debt and equity markets in crisis countries function as main transmission channels of the crisis. Bekiros et al. (2018) utilize a dynamic copula approach to analyze the time-varying dependence structure between the European government bond markets around the Eurozone debt crisis.

\subsection{Integration and co-movement in government bond markets}

Another strand of related literature focuses on integration and co-movement in international government bond markets. ${ }^{3}$ The major part of this literature pertains to the developed bond markets, mainly in the Eurozone and G-7 economies (e.g., Kumar and Okimoto, 2011; Abad et al., 2014; Christiansen 2014). The broad conclusion from those studies is reflected in the evidence of the timevarying nature of the government bond market integration, with the degree of integration decreasing after the beginning of the global financial crisis. Similarly, Ehrmann and Fratzscher (2017) provide evidence that the level of integration in Eurozone government bond markets has decreased due to the sovereign debt crisis in 2010. Chaieb et al. (2020), analyzing 21 developed and 18 emerging countries, report that the degree of sovereign bond integration and dynamics is very heterogeneous. Similarly, Abakah et al. (2021) examine co-movement dynamics between nine developed bond markets by using copula modelling and find evidence that magnitude of the dependence is heterogeneous across bond markets.

A group of studies on bond market integration is devoted to emerging and frontier bond markets. For instance, Kim et al. (2006) focus on the dynamic bond market linkages in a subset of European emerging markets. Bunda et al. (2009) investigate bond markets' co-movement in a large group of emerging markets. Piljak (2013) examines the time-varying co-movement of frontier and emerging government bond markets with the US market. Piljak and Swinkels (2017a) provide a comprehensive analysis of frontier government bond markets co-movement dynamics relative to emerging bond markets, the US corporate bond market, and the US Treasury market. Their principal findings regarding emerging markets are supporting the time-varying nature of correlations with developed markets. Frontier markets appear to be less integrated, thus providing diversification potential for international investors. Agur et al. (2019)

${ }^{3}$ For a detailed review of literature on international financial integration, see Lucey et al. (2018). 
find that for emerging market US dollar-denominated debt markets are more internationally integrated than local currencydenominated debt markets. Furthermore, Šimović et al. (2016) provide evidence that the integration of sovereign bond markets in European post-transition economies with the Eurozone bond market decreased during the financial crisis.

\subsection{Stock-bond correlations and flight-to-quality}

The literature on stock-bond correlations is extensive and covers different aspects of correlation modelling (e.g., De Goeij and Marquering, 2004; Cappiello et al., 2006; Aslanidis and Christiansen, 2014), determinants of stock and bond return co-movement (Yang et al., 2009; Baele et al., 2010), and flights-to-safety (Baur and Lucey, 2009; Brière et al., 2012; Baele et al, 2020). The early studies in this stream concentrated on developed markets (e.g., Campbell and Ammer, 1993; Connolly et al., 2005; d'Addona and Kind, 2006). In particular, Connolly et al. (2005) examine stock-bond correlations with respect to stock market uncertainty, while d'Addona and Kind (2006) utilize affine asset pricing model to jointly value stocks and bonds and document correlations in post war period in the sample of seven developed countries. The scope of the literature has been extended during the last decade to cover also emerging markets (Li and Zou, 2008; Panchenko and Wu, 2009; Christopher et al., 2012; Bianconi et al., 2013; Dimic et al., 2016). The issue of stock-bond correlations in emerging markets is mainly examined by looking at co-movement of stocks and bonds within individual emerging countries and analyzing factors that affect co-movement, such as policy and information shocks (Li and Zou, 2008), sovereign credit ratings (Christopher et al., 2012), the US financial stress (Bianconi et al., 2013), macroeconomic fundamentals and market uncertainty (Dimic et al., 2016), and stock market integration (Panchenko and Wu, 2009).

One specific part of the stock-bond literature focuses on the "flight-to-safety" phenomenon characterized by a negative stock-bond correlations during crisis periods, which lead to potential diversification benefits. The most notable early contributions among others are Gulko (2002) who analyzes "decoupling effect", and Hartmann et al. (2004) who document that flight-to-quality from stocks into bonds has approximately similar frequency as stock-bond contagion. Baur and Lucey (2009) go further and propose a definition and a reliant test to address the flight-to-quality and cross-asset contagion phenomena. Their empirical analysis of eights developed markets uncovers the presence of flight-to-quality in the intersection of stock and bond markets, which is especially pronounced during crisis periods. Brière et al. (2012) document existence of flight-to-quality and instability of correlations. Baele et al. (2020) establish the existence of flight-to-quality episodes in all of the twenty-three countries they examined.

\section{Data}

Our dataset consists of weekly data for different indices classified into three categories of government bond markets: developed, emerging, and frontier. We use total return indices, meaning that they incorporate both bond price changes and coupon payments. The sample period spans from 10 May 2002 to 29 December 2017.

The category of emerging markets encompasses three different subcategories: (i) USD-denominated government bonds represented by the JP Morgan Emerging Markets Bond Index Plus (EMBI+), (ii) local currency government bonds with returns hedged to the USD using currency derivatives, and (iii) local currency government bonds converted to USD without currency hedging. ${ }^{4}$ The EMBI+ is a commonly used sovereign bond emerging market benchmark, which tracks the performance of USD-denominated debt instruments issued by governments of emerging markets, and it includes Brady bonds, loans, and Eurobonds. The local currency government bonds are proxied by the JP Morgan Government Bond Index- Emerging Markets (GBI-EM). ${ }^{5}$ For GBI-EM, we also use subindexes for different maturities: short (1-5 years), medium (5-10 years), and long term (15+ years).

The frontier government bond markets are captured with the JP Morgan Next Generation Markets Index (NEXGEM), which tracks USD-denominated government debt issued by frontier markets. The frontier markets group consists of smaller and less liquid markets that are called "next-generation issuers" since they are considered as the next wave of emerging markets. The requirement for classification into the frontier group is that the country must have a rating of Ba1/BB + or lower by both Moody's and S\&P. At the same time, inclusion criteria require that bonds have a current face amount outstanding of at least USD 500 million and remaining maturity longer than 2,5 years. The frontier markets have lower ratings than traditional emerging markets, but due to their usual higher yields and less integrated economies, they represent an alternative investment opportunity and a potential source of additional diversification benefits (see, Piljak and Swinkels, 2017a). Inevitably, the indexes that we use occasionally change country composition over time, as countries are added when they issue qualifying bonds or removed when they no longer have qualifying bonds outstanding. Therefore, to thoroughly capture all of their movements in addition to the aggregate level indices, for emerging and frontier markets, we also perform analysis on the regional and country level. ${ }^{6}$

These fixed income asset classes are accessible to investors around the world through exchange traded funds or mutual funds. For

\footnotetext{
${ }^{4}$ The link below points to more detailed description of all JP Morgan indices we use in our study. https://www.jpmorgan.com/country/US/EN/ $\underline{\text { jpmorgan/investbk/solutions/research/indices/product }}$

5 https://www.jpmorgan.com/jpmpdf/1320696554395.pdf

6 The NEXGEM has four regional indices (Africa, Asia, Latin America, and Middle East), while EMBI+ has regional coverage of Africa, Asia, Latin America, and Europe. For country level analysis of frontier markets we include countries that have sufficient period of data availability without time breaks (Dominican Republic, Ecuador, Egypt, and Pakistan), while for emerging markets we focus on countries that have relatively higher country weights in the EMBI+ composition and longer periods of data availability (Brazil, Colombia, Mexico, Panama, Peru, Philippines, Russia, South Africa, and Turkey).
} 
EMBI+

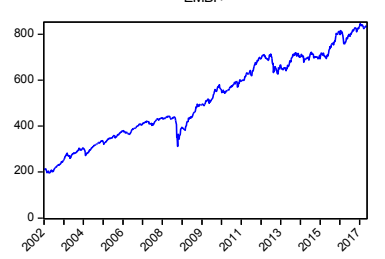

France- No Hedge

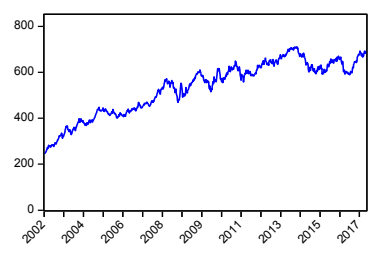

Japan - No Hedge

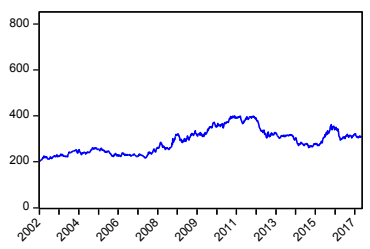

us

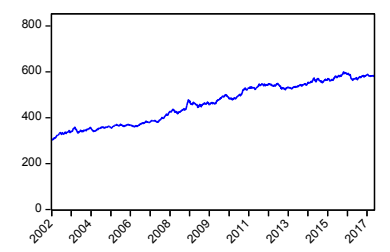

NEXGEM

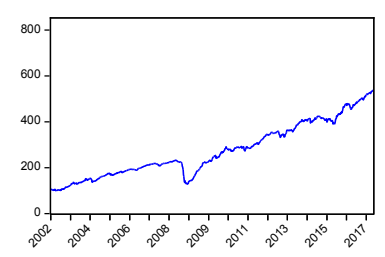

France - Hedge

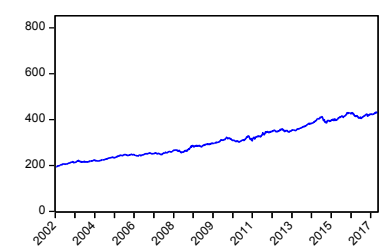

Japan- Hedge

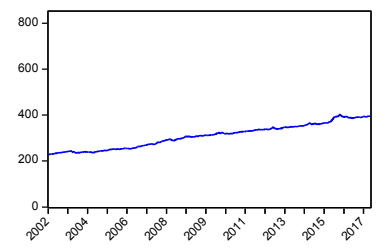

GABI

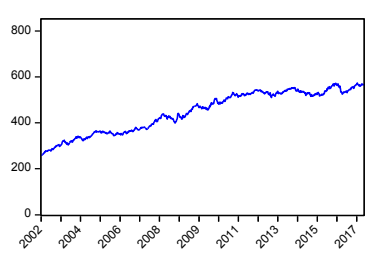

GBI EM - No Hedged

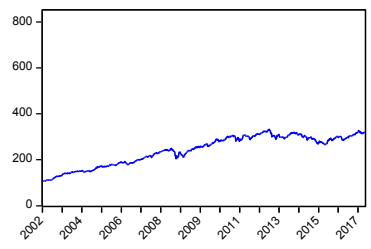

Germany- № Hedge

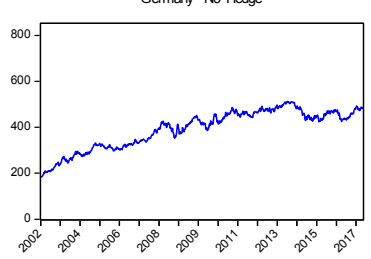

UK - No Hedge

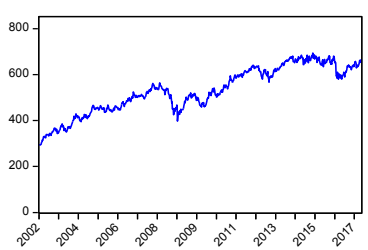

GBI EM - Hedged

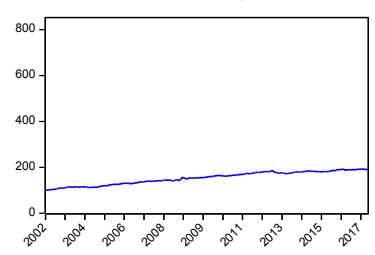

Germany - Hedge

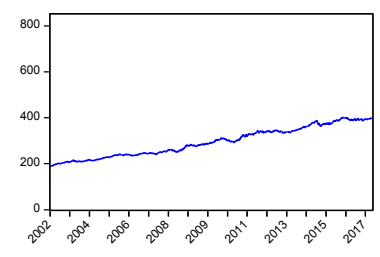

UK - Hedge

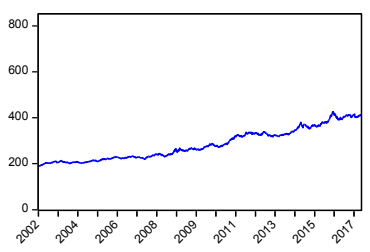

Fig. 1. Time evolution of the bond indices. This figure shows the evolution of bond indices over the sample period (10th May $2002-29$ th December 2017). All indices and their corresponding categories of markets (emerging, frontier, and developed) are described in Section 3 of the paper.

example, the 'iShares J.P. Morgan USD Emerging Markets Bond ETF' (Ticker: EMB) can be used to access USD-denominated emerging markets, 'SPDR Barclays Capital Emerging Markets Local Bond ETF' (Ticker: EBND) for local-currency emerging markets bonds, and the 'NN Frontier Markets Debt (Hard Currency) Fund' (ISIN: LU0990547431) for frontier bond markets. These are illustrations of funds, but investors have more options, depending on their geographical location. Our insights can also benefit portfolio managers of fixed income funds who manage portfolios by allocating to individual bonds and can choose between different types of bonds that we analyze in this paper.

Our dataset also includes a global bond market portfolio, which is proxied by the JP Morgan Global Aggregate Bond Index (GABI). We choose the GABI as our global benchmark constructed combining 5500 instruments issued in more than 60 countries with the denomination in over 25 currencies, and its total market value averaging about USD 20 trillion.

The developed markets are proxied by JP Morgan Government Bond Index Broad (GBI Broad), which tracks bond issuances from high-income countries worldwide. For GBI Broad, we also use subindexes for different maturities: short (1-5 years), medium (5-10 years), and long term (15 + years). We consider returns converted to a common currency, the US dollar (USD) in our case, and returns hedged to USD using currency derivatives. Analyzing both currency-hedged and open currency returns enables us to examine the effect of exchange rates on international bond returns. Provided that most of the constituents of GBI Broad are also constituents of our benchmark for the global bond market (GABI), we select France, Germany, Japan, the UK, and the US as representatives of developed markets subsample. Consequently, we report country-level instead of aggregate-index level analysis for the subsample of developed markets.

Fig. 1 shows the evolution of the bond indices over time. In general, the indices of emerging, frontier and developed markets exhibit the pattern of upward moving trend over time constituting co-movement with the global bond market benchmark (GABI). The USDdenominated emerging (EMBI+) and frontier markets (NEXGEM) show sharp drop in value during the global financial crisis, which is consistent with "equity-like" properties of emerging and frontier bonds. The local currency denominated emerging markets bond index GBI EM (unhedged returns) shows also slight drop in value (but of the smaller magnitude) at the same time, while on the contrary localcurrency emerging markets bond index GBI EM (with returns hedged to the USD using currency derivatives) exhibit very little 
Table 1

Descriptive statistics.

\begin{tabular}{|c|c|c|c|c|c|c|c|c|c|}
\hline & Mean & Median & Max & Min & St. Dev. & Skewness & Kurtosis & JB stat. & p-Value \\
\hline \multicolumn{10}{|c|}{ Panel A: Emerging Markets } \\
\hline EMBI + & 0.0017 & 0.0022 & 0.1280 & -0.1487 & 0.0134 & -2.0295 & 41.7133 & 51327.11 & 0.0000 \\
\hline GBI EM - No Hedge & 0.0014 & 0.0020 & 0.0549 & -0.0652 & 0.0109 & -0.6918 & 7.4748 & 747.71 & 0.0000 \\
\hline GBI EM - Hedged & 0.0008 & 0.0010 & 0.0208 & -0.0213 & 0.0035 & -0.2739 & 8.9460 & 1215.25 & 0.0000 \\
\hline \multicolumn{10}{|c|}{ Panel B: Frontier Markets } \\
\hline NEXGEM & 0.0020 & 0.0026 & 0.0546 & -0.1582 & 0.0137 & -3.4781 & 37.1412 & 41023.37 & 0.0000 \\
\hline \multicolumn{10}{|c|}{ Panel C: Developed Markets } \\
\hline \multicolumn{10}{|c|}{ Not Hedged } \\
\hline France & 0.0013 & 0.0018 & 0.0600 & -0.0532 & 0.0146 & -0.1759 & 3.6558 & 18.875 & 0.0001 \\
\hline Germany & 0.0012 & 0.0015 & 0.0600 & -0.0487 & 0.0142 & -0.1396 & 3.5444 & 12.759 & 0.0017 \\
\hline Japan & 0.0005 & 0.0004 & 0.0896 & -0.0595 & 0.0153 & 0.2968 & 4.7995 & 122.374 & 0.0000 \\
\hline UK & 0.0010 & 0.0016 & 0.0671 & -0.1165 & 0.0154 & -0.7879 & 7.9364 & 915.192 & 0.0000 \\
\hline US & 0.0008 & 0.0010 & 0.0257 & -0.0209 & 0.0063 & -0.2952 & 3.7025 & 28.702 & 0.0000 \\
\hline \multicolumn{10}{|l|}{ Hedged } \\
\hline France & 0.0010 & 0.0014 & 0.0283 & -0.0237 & 0.0061 & -0.2552 & 4.7447 & 112.627 & 0.0000 \\
\hline Germany & 0.0009 & 0.0014 & 0.0222 & -0.0228 & 0.0058 & -0.2926 & 3.8267 & 34.967 & 0.0000 \\
\hline Japan & 0.0007 & 0.0008 & 0.0181 & -0.0159 & 0.0031 & -0.2996 & 6.5643 & 445.243 & 0.0000 \\
\hline UK & 0.0009 & 0.0012 & 0.0436 & -0.0332 & 0.0086 & -0.1303 & 4.3405 & 63.561 & 0.0000 \\
\hline \multicolumn{10}{|c|}{ anel D: Global bond ma } \\
\hline \multicolumn{10}{|c|}{ Panel D: Global Bond Market Benchmark } \\
\hline GABI & 0.0009 & 0.0009 & 0.0333 & -0.0272 & 0.0079 & -0.0910 & 3.5700 & 12.204 & 0.0022 \\
\hline \multicolumn{10}{|c|}{ Panel E: Global Stock Market Benchmark } \\
\hline MSCI AC World & 0.0014 & 0.0034 & 0.1172 & -0.2235 & 0.0237 & -1.3507 & 15.1009 & 5239.646 & 0.0000 \\
\hline
\end{tabular}

This table reports descriptive statistics for weekly index returns for emerging, frontier, and developed markets from May 10, 2002, to December 29, 2017. A total number of weekly observations is 818 for each category of markets. Panel A contains the returns of USD-denominated emerging markets government bonds (EMBI + ) and local currency emerging markets government bonds (returns hedged to US dollar using currency derivatives and returns without currency hedging). Panel B contains the returns of USD-denominated frontier government bonds (NEXGEM), while Panel C contains returns for developed government bond markets (returns hedged to US dollar using currency derivatives and returns without currency hedging). Panel D reports returns for global bond market benchmark (GABI), while Panel E shows returns for global stock market benchmark (MSCI All Country World Equity Index).

variability during the crisis and over entire sample period. A similar pattern regarding difference between hedged and unhedged returns is also observed across developed markets.

Table 1 displays descriptive statistics of weekly bond index returns for all indices. ${ }^{7}$ The statistics show that the highest average weekly return among our three observed categories of the markets is at the level of $0.20 \%$ and for the group of frontier markets. The observed return in frontier markets is slightly higher than the $0.18 \%$ average return on USD-denominated emerging market index. The average weekly return on local currency emerging market index in the case of USD-hedged returns is $0.08 \%$, which is almost on the same level as the USD-hedged returns on developed markets. Also, frontier markets appear to be the most volatile ones, closely followed by USD-denominated emerging market index. Table 1 also includes descriptive statistics for the MSCI All Country World Equity Index in US dollars, which is used to represent the global stock market used in the stock-bond correlation analysis.

\section{Methodology}

Utilizing the quantile-based methodology proposed by Baur (2013), we analyze the degree and the structure of the dependences of global government bond markets. ${ }^{8}$ The base quantile regression model is in the following form:

$$
Q_{r_{i}}(\tau \mid \mathbf{X})=\alpha(\tau)+\beta(\tau) r_{M}+\gamma_{1}(\tau) r_{M} D_{\text {fincrisis }}+\gamma_{2}(\tau) r_{M} D_{\text {debtcrisis }}+\delta(\tau) V I X
$$

where $r_{i}$ denotes the returns of government bond market $i ; r_{m}$ is the return on global bond market portfolio; $Q_{r_{i}}(\tau \mid \mathbf{X})$ denotes the $\tau_{\text {th }}$ conditional quantile of $r_{i}$ given the explanatory variables denoted with X;. $D_{\text {fincrisis }}$ is a dummy variable capturing the global financial crisis period; $D_{\text {debtcrisis }}$ is a dummy variable capturing the Eurozone debt crisis period; and VIX refers to global market uncertainty proxied by the volatility index VIX. ${ }^{9}$

Each quantile represents a different state of the government bond returns, where for instance, lower quantiles (e.g., 2nd quantile)

\footnotetext{
${ }^{7}$ Descriptive statistic for regional and country level for emerging and frontier markets is given in Appendix A (Table A.1).

${ }^{8}$ Koenker and Hallock (2001) offer a comprehensive overview of the quantile regression and its application in finance and economics research. Boubaker et al. (2019) provide discussion on advantages of quantile regression in examining government bond markets.

9 The VIX Index is used as a control variable in the model. We tested also the MOVE Index (the Merrill Lynch Option Volatility Estimated Index) as a proxy for bond market uncertainty and the results are qualitatively similar to the ones obtained by using VIX.
} 
are interpreted as a bad state and higher quantiles (e.g. 98th quantile) as a good state. If the Equation (1) would be specified without crises dummy variables, then the degree and structure of dependence would be conditional on the state of the government bond return $\left(r_{i}\right)$ and it would be entirely captured by $\beta(\tau)$. The role of the interaction term $\gamma_{1}(\tau) r_{M} D_{\text {fincrisis }}$ in Equation (1) is to capture differences in the degree and structure of dependence between normal time and global financial crisis time. Similarly, the role of the interaction term $\gamma_{2}(\tau) r_{M} D_{\text {debtcrisis }}$ is to capture differences in the degree and structure of dependence between normal time and the Eurozone debt crisis time. Hence, the model specification provided in Equation (1) enables examining whether there is crisis-specific change in the degree and structure of dependence. The $D_{\text {fincrisis }}$ is a dummy variable equal to one for observations in the period between 3 August 2007 until 26 December 2008, which constitutes the global financial crisis, and it is zero otherwise; and similarly $D_{\text {debtcrisis }}$ is a dummy variable equal to one for observations in the period of the Eurozone debt crisis, from 2 October 2009 to 27 July 2012 and equal to zero otherwise. $^{10}$

The estimated quantile regression coefficients determine the dependence relationship between the global bond market returns $\left(r_{m}\right)$ and $\tau_{\text {th }}$ conditional quantile of emerging, frontier, and developed bond markets returns $\left(r_{i}\right)$. The complete dependence structure is determined by the pattern of the estimated coefficients across all quantiles. If estimated coefficients do not change across quantiles, then the dependence structure is constant. By contrast, if estimated coefficients for low and high quantiles are statistically different from each other, then the observed structure is asymmetric.

In addition to the structure of dependence, quantile regression also enables determining the degree of dependence (defined as the average over 99 quantile regression coefficients). Specifically, the degree of dependence in the non-crisis period is average of the terms $\beta(\tau)$, while the change in the degree of dependence due to the global financial crisis is average of the terms $\gamma_{1}(\tau)$ and the change in the degree of dependence due to the Eurozone debt crisis is average of the terms $\gamma_{2}(\tau)$.

\section{Empirical results}

In this section, we report empirical results of the structure and degree of dependence in international government bond markets. In the Sections 5.1, 5.2, and 5.3 we describe the estimation results of the quantile regression given in Equation (1). Tables 2-4 report the coefficient estimates of $\beta, \gamma_{1}$, and $\gamma_{2}$ respectively for selected quantiles and the degree of dependence for emerging (Panel A), frontier (Panel B), and developed markets (Panel C). ${ }^{11}$ The parameter $\gamma_{1}$ models the change of the dependence during the global financial crisis period, while $\gamma_{2}$ models the change of the dependence during the Eurozone debt crisis period. The aggregate or full effect for a specific quantile in the global financial crisis period is calculated as the summation of the coefficient estimates $\beta$ and $\gamma_{1}$. Similarly, the aggregate effect for a specific quantile in the Eurozone debt crisis period is given by the sum of coefficient estimates $\beta$ and $\gamma_{2}$. Figs. 2 and 3 provide a graphical illustration of the dependence structure and changes in the dependence structure associated with the global financial crisis and Eurozone debt crisis. In Section 5.4 we display the results of the test for inter-quantile differences (Table 5) and analysis of asymmetry (Table 6). In Section 5.5 we show results from our analysis on bond maturity segments (Table 7 and Fig. 4 ). Finally, in Section 5.6 we report results on contagion and flights from global stock to bond markets (Table 8), while in Section 5.7 we provide portfolio management application (Table 9). To facilitate interpretation of the results from the currency aspect, in the following three sub-sections, we present the results separately for local-currency emerging markets, USD-denominated emerging and frontier markets, and developed markets.

\subsection{Dependence in local-currency emerging bond markets}

Emerging markets local currency unhedged returns have a different dependence pattern from emerging markets hedged returns (as shown in Fig. 2, Panel A). In particular, the dependence pattern for emerging markets hedged return is almost constant over all quantiles and during both crises periods; while emerging markets local currency unhedged returns have an asymmetric dependence pattern in the left and right tails. The documented patterns suggest that the relation between hedged returns of emerging market bonds and the global bond market is close to 0.2 , and does not depend on the extremity of returns or whether there is a crisis period. Reported absence of volatility of hedged returns agrees with prior findings in the literature (Burger and Warnock, 2007). Finally, it is aligned with Jeanneret and Souissi's (2016) results that governments default for a different reason on domestic and foreign debt and that global risk factors drive the dynamics of sovereign risk. Our observed result holds only for the interest rate component of the bond return. However, as for the unhedged returns, the beta coefficient is close to 0.8 (left tail) and 0.6 (right tail), and during both crisis periods, the dependencies shoot up in the left tail. Only during the global financial crisis, the right-tail dependence shoots down. This observed pattern indicates that during the crises emerging markets currency returns move more together with the global bond market index than emerging local currency bond returns.

Further examination of the degree of dependence shows that its level in normal times (average of $\beta$ coefficients across all quantiles) is around 0.68 for unhedged returns, vs. approximately 0.15 for hedged returns. This evidence indicates substantial variation in the

\footnotetext{
10 For the global financial crisis, we use the same timeline as Baur (2013), which is based on the timeline given by Federal Reserve Board of St. Louis (2010) and the Bank for International Settlements (BIS; see Filardo et al., 2010). For the Eurozone debt crisis, the choice of the beginning date of the Eurozone debt crisis is the time when the Greek Prime Minister, George Papandreou, announced Greece's severe fiscal problems. The end of the crisis period is chosen when Mario Draghi, the president of the ECB, disclosed publicly that "the ECB is ready to do whatever it takes to preserve the Euro" (see Bloomberg, 2012). This timeline is also in accordance with Lane (2012).

11 The results for coefficient estimates of $\delta$ (VIX Index) are not reported here for sake of brevity, but are available from the authors upon request.
} 
Table 2

Structure and degree of the dependence.

\begin{tabular}{|c|c|c|c|c|c|c|c|c|c|c|c|c|}
\hline Quantile & 1 & 2 & 5 & 10 & 25 & 50 & 75 & 90 & 95 & 98 & 99 & Degree \\
\hline \multicolumn{13}{|c|}{ Panel A: Emerging Markets } \\
\hline EMBI + & $\begin{array}{l}0.815^{* * *} \\
(5.97)\end{array}$ & $\begin{array}{l}0.585^{* * *} \\
(8.61)\end{array}$ & $\begin{array}{l}0.640^{* * * *} \\
(7.34)\end{array}$ & $\begin{array}{l}0.589^{* * *} \\
(9.59)\end{array}$ & $\begin{array}{l}0.478^{* * *} \\
(6.99)\end{array}$ & $\begin{array}{l}0.457^{* * *} \\
(7.39)\end{array}$ & $\begin{array}{l}0.477^{* * * *} \\
(8.34)\end{array}$ & $\begin{array}{l}0.502^{* * *} \\
(6.42)\end{array}$ & $\begin{array}{l}0.554^{* * *} \\
(4.92)\end{array}$ & $\begin{array}{l}0.657^{* * * *} \\
(5.57)\end{array}$ & $\begin{array}{l}0.640^{* * *} \\
(6.43)\end{array}$ & 0.516 \\
\hline EM Local - No Hedge & $\begin{array}{l}0.813^{* * *} \\
(5.25)\end{array}$ & $\begin{array}{l}0.690^{* * *} \\
(6.34)\end{array}$ & $\begin{array}{l}0.809^{* * *} \\
(10.91)\end{array}$ & $\begin{array}{l}0.770^{* * *} \\
(10.47)\end{array}$ & $\begin{array}{l}0.748^{* * *} \\
(12.64)\end{array}$ & $\begin{array}{l}0.640^{* * *} \\
(11.98)\end{array}$ & $\begin{array}{l}0.623^{* * *} \\
(11.43)\end{array}$ & $\begin{array}{l}0.650^{* * * *} \\
(8.91)\end{array}$ & $\begin{array}{l}0.626^{\text {**** }} \\
(6.04)\end{array}$ & $\begin{array}{l}0.591 * * * \\
(5.15)\end{array}$ & $\begin{array}{l}0.587^{* * *} \\
(5.97)\end{array}$ & 0.689 \\
\hline EM Local - Hedge & $\begin{array}{l}0.346^{* * *} \\
(3.55)\end{array}$ & $\begin{array}{l}0.190^{* * *} \\
(5.80)\end{array}$ & $\begin{array}{l}0.233^{* * *} \\
(7.65)\end{array}$ & $\begin{array}{l}0.220^{* * *} \\
(6.18)\end{array}$ & $\begin{array}{l}0.161^{* * *} \\
(8.77)\end{array}$ & $\begin{array}{l}0.139^{* * *} \\
(8.32)\end{array}$ & $\begin{array}{l}0.135^{* * *} \\
(5.89)\end{array}$ & $\begin{array}{l}0.122^{* * * *} \\
(4.68)\end{array}$ & $\begin{array}{l}0.069^{* * *} \\
(2.03)\end{array}$ & $\begin{array}{l}0.141^{*} \\
(1.65)\end{array}$ & $\begin{array}{l}0.253^{* * * *} \\
(3.25)\end{array}$ & 0.157 \\
\hline \multicolumn{13}{|c|}{ Panel B: Frontier Markets } \\
\hline Frontier & $\begin{array}{l}0.709^{* * *} \\
(2.64)\end{array}$ & $\begin{array}{l}0.341^{* *} \\
(2.36)\end{array}$ & $\begin{array}{l}0.214^{* *} \\
(2.11)\end{array}$ & $\begin{array}{l}0.153^{* *} \\
(2.27)\end{array}$ & $\begin{array}{c}0.045 \\
(0.57)\end{array}$ & $\begin{array}{c}0.079 \\
(1.58)\end{array}$ & $\begin{array}{l}-0.012 \\
(-0.21)\end{array}$ & $\begin{array}{c}0.056 \\
(0.69)\end{array}$ & $\begin{array}{c}0.147 \\
(1.24)\end{array}$ & $\begin{array}{c}0.162 \\
(1.36)\end{array}$ & $\begin{array}{c}0.048 \\
(0.35)\end{array}$ & 0.094 \\
\hline \multicolumn{13}{|c|}{ Panel C: Developed Markets } \\
\hline France - No Hedge & $\begin{array}{l}1.879 * * * \\
(17.42)\end{array}$ & $\begin{array}{l}1.854^{* * *} \\
(18.95)\end{array}$ & $\begin{array}{l}1.715^{* * *} \\
(27.77)\end{array}$ & $\begin{array}{l}1.725^{* * *} \\
(28.79)\end{array}$ & $\begin{array}{l}1.688^{* * *} \\
(46.36)\end{array}$ & $\begin{array}{l}1.573^{* * *} \\
(48.74)\end{array}$ & $\begin{array}{l}1.546^{* * *} \\
(36.48)\end{array}$ & $\begin{array}{l}1.512^{* * *} \\
(23.10)\end{array}$ & $\begin{array}{l}1.511^{* * *} \\
(19.13)\end{array}$ & $\begin{array}{l}1.445^{* * *} \\
(14.00)\end{array}$ & $\begin{array}{l}1.437^{* * * *} \\
(12.49)\end{array}$ & 1.611 \\
\hline Germany -No Hedge & $\begin{array}{l}1.917^{* * * *} \\
(17.90)\end{array}$ & $\begin{array}{l}1.769^{* * *} \\
(19.47)\end{array}$ & $\begin{array}{l}1.699^{* * *} \\
(32.10)\end{array}$ & $\begin{array}{l}1.750^{* * * *} \\
(38.16)\end{array}$ & $\begin{array}{l}1.684^{* * *} \\
(41.32)\end{array}$ & $\begin{array}{l}1.597^{* * * *} \\
(46.92)\end{array}$ & $\begin{array}{l}1.541^{* * * *} \\
(37.06)\end{array}$ & $\begin{array}{l}1.533^{* * *} \\
(35.78)\end{array}$ & $\begin{array}{l}1.474^{* * *} \\
(16.41)\end{array}$ & $\begin{array}{l}1.452^{* * * *} \\
(31.92)\end{array}$ & $\begin{array}{l}1.488^{* * * *} \\
(27.44)\end{array}$ & 1.608 \\
\hline Japan - No Hedge & $\begin{array}{l}1.615^{* * * *} \\
(14.35)\end{array}$ & $\begin{array}{l}1.411^{* * *} \\
(9.76)\end{array}$ & $\begin{array}{l}1.467^{* * *} \\
(14.79)\end{array}$ & $\begin{array}{l}1.455^{* * * *} \\
(16.08)\end{array}$ & $\begin{array}{l}1.387^{* * *} \\
(18.95)\end{array}$ & $\begin{array}{l}1.257^{* * *} \\
(21.21)\end{array}$ & $\begin{array}{l}1.381^{* * * *} \\
(21.26)\end{array}$ & $\begin{array}{l}1.447^{* * * *} \\
(16.08)\end{array}$ & $\begin{array}{l}1.580^{* * *} \\
(19.14)\end{array}$ & $\begin{array}{l}1.625^{* * *} \\
(20.17)\end{array}$ & $\begin{array}{l}1.552^{* * * *} \\
(22.28)\end{array}$ & 1.371 \\
\hline UK - No Hedge & $\begin{array}{l}1.993^{* * *} \\
(6.68)\end{array}$ & $\begin{array}{l}1.713^{* * *} \\
(14.32)\end{array}$ & $\begin{array}{l}1.657^{* * *} \\
(20.29)\end{array}$ & $\begin{array}{l}1.563^{* * * *} \\
(13.74)\end{array}$ & $\begin{array}{l}1.498^{* * *} \\
(21.81)\end{array}$ & $\begin{array}{l}1.436^{* * *} \\
(24.77)\end{array}$ & $\begin{array}{l}1.424^{* * *} \\
(19.51)\end{array}$ & $\begin{array}{l}1.381^{* * *} \\
(20.56)\end{array}$ & $\begin{array}{l}1.364^{* * *} \\
(14.98)\end{array}$ & $\begin{array}{l}1.270^{* * *} \\
(8.66)\end{array}$ & $\begin{array}{l}1.065^{* * * *} \\
(7.72)\end{array}$ & 1.468 \\
\hline US - No Hedge & $\begin{array}{l}0.379^{* * * *} \\
(5.87)\end{array}$ & $\begin{array}{l}0.509^{* * *} \\
(7.95)\end{array}$ & $\begin{array}{l}0.579^{* * *} \\
(13.48)\end{array}$ & $\begin{array}{l}0.611^{* * * *} \\
(17.58)\end{array}$ & $\begin{array}{l}0.575^{* * *} \\
(21.02)\end{array}$ & $\begin{array}{l}0.587^{* * *} \\
(19.11)\end{array}$ & $\begin{array}{l}0.484^{* * *} \\
(15.86)\end{array}$ & $\begin{array}{l}0.434^{* * *} \\
(12.25)\end{array}$ & $\begin{array}{l}0.417^{* * *} \\
(11.09)\end{array}$ & $\begin{array}{l}0.381^{* * *} \\
(6.84)\end{array}$ & $\begin{array}{l}0.343^{* * * *} \\
(7.94)\end{array}$ & 0.541 \\
\hline France - Hedged & $\begin{array}{l}0.836^{* * * *} \\
(12.45)\end{array}$ & $\begin{array}{l}0.681^{* * *} \\
(4.04)\end{array}$ & $\begin{array}{l}0.491^{* * *} \\
(4.46)\end{array}$ & $\begin{array}{l}0.447^{* * *} \\
(15.78)\end{array}$ & $\begin{array}{l}0.414^{* * *} \\
(16.71)\end{array}$ & $\begin{array}{l}0.354^{* * * *} \\
(11.77)\end{array}$ & $\begin{array}{l}0.296^{* * *} \\
(8.25)\end{array}$ & $\begin{array}{l}0.236^{* * *} \\
(4.47)\end{array}$ & $\begin{array}{l}0.165^{* * *} \\
(3.53)\end{array}$ & $\begin{array}{l}0.205^{* * *} \\
(2.66)\end{array}$ & $\begin{array}{l}0.320^{* * * *} \\
(2.72)\end{array}$ & 0.357 \\
\hline Germany - Hedged & $\begin{array}{l}0.723^{* * *} \\
(8.56)\end{array}$ & $\begin{array}{l}0.587^{* * *} \\
(3.06)\end{array}$ & $\begin{array}{l}0.510^{* * * *} \\
(6.88)\end{array}$ & $\begin{array}{l}0.445^{* * * *} \\
(8.03)\end{array}$ & $\begin{array}{l}0.407^{* * *} \\
(15.80)\end{array}$ & $\begin{array}{l}0.379^{* * *} \\
(12.48)\end{array}$ & $\begin{array}{l}0.275^{* * * *} \\
(8.13)\end{array}$ & $\begin{array}{l}0.224^{* * * *} \\
(5.50)\end{array}$ & $\begin{array}{l}0.195^{* * *} \\
(4.21)\end{array}$ & $\begin{array}{l}0.144^{* * *} \\
(2.01)\end{array}$ & $\begin{array}{l}-0.021 \\
(-0.22)\end{array}$ & 0.351 \\
\hline Japan - Hedged & $\begin{array}{l}0.293^{* * * *} \\
(5.51)\end{array}$ & $\begin{array}{l}0.241^{* * *} \\
(5.73)\end{array}$ & $\begin{array}{l}0.208^{* * *} \\
(6.80)\end{array}$ & $\begin{array}{l}0.168^{* * *} \\
(7.68)\end{array}$ & $\begin{array}{l}0.135^{* * *} \\
(8.10)\end{array}$ & $\begin{array}{l}0.105^{* * *} \\
(6.38)\end{array}$ & $\begin{array}{l}0.104^{* * * *} \\
(6.36)\end{array}$ & $\begin{array}{l}0.113^{* * *} \\
(3.64)\end{array}$ & $\begin{array}{l}0.145^{* * * *} \\
(4.49)\end{array}$ & $\begin{array}{l}0.203^{* * * *} \\
(3.16)\end{array}$ & $\begin{array}{l}0.177^{* *} \\
(2.00)\end{array}$ & 0.131 \\
\hline UK - Hedged & $\begin{array}{l}0.576^{* * *} \\
(3.06)\end{array}$ & $\begin{array}{l}0.707 * * * \\
(5.19)\end{array}$ & $\begin{array}{l}0.581^{* * * *} \\
(9.34)\end{array}$ & $\begin{array}{l}0.640^{* * *} \\
(12.97)\end{array}$ & $\begin{array}{l}0.578^{* * * *} \\
(13.89)\end{array}$ & $\begin{array}{l}0.573^{* * *} \\
(12.49)\end{array}$ & $\begin{array}{l}0.546^{* * * *} \\
(9.51)\end{array}$ & $\begin{array}{l}0.590 * * * \\
(7.28)\end{array}$ & $\begin{array}{l}0.571^{* * * *} \\
(5.38)\end{array}$ & $\begin{array}{l}0.392^{* * *} \\
(3.85)\end{array}$ & $\begin{array}{l}0.474 * * * \\
(3.34)\end{array}$ & 0.563 \\
\hline
\end{tabular}

This table reports the quantile regression coefficient estimates of $\beta$ for the variable $r_{m}$ (the return on the global bond market portfolio) according to the model defined by Equation (1). T-statistics are in parentheses. The last column shows degree of dependence. 
Table 3

Structure and degree of the dependence (change during the global financial crisis period).

\begin{tabular}{|c|c|c|c|c|c|c|c|c|c|c|c|c|}
\hline Quantile & 1 & 2 & 5 & 10 & 25 & 50 & 75 & 90 & 95 & 98 & 99 & Degree \\
\hline \multicolumn{13}{|c|}{ Panel A: Emerging Markets } \\
\hline EMBI + & $\begin{array}{l}1.192^{* * *} \\
(9.45)\end{array}$ & $\begin{array}{l}1.309^{* * *} \\
(10.92)\end{array}$ & $\begin{array}{c}0.490 \\
(0.89)\end{array}$ & $\begin{array}{c}0.002 \\
(0.01)\end{array}$ & $\begin{array}{l}-0.061 \\
(-0.40)\end{array}$ & $\begin{array}{l}-0.302^{* *} \\
(-2.50)\end{array}$ & $\begin{array}{c}0.011 \\
(0.06)\end{array}$ & $\begin{array}{c}0.224 \\
(1.45)\end{array}$ & $\begin{array}{l}-0.170 \\
(-0.82)\end{array}$ & $\begin{array}{l}-0.504 \\
(-1.53)\end{array}$ & $\begin{array}{l}-1.651^{* * *} \\
(-10.81)\end{array}$ & -0.107 \\
\hline EM Local - No Hedge & $\begin{array}{l}0.526^{*} \\
(1.76)\end{array}$ & $\begin{array}{c}0.452 \\
(0.73)\end{array}$ & $\begin{array}{l}-0.159 \\
(-0.13)\end{array}$ & $\begin{array}{l}-0.046 \\
(-0.13)\end{array}$ & $\begin{array}{l}-0.165 \\
(-0.64)\end{array}$ & $\begin{array}{l}-0.076 \\
(-0.58)\end{array}$ & $\begin{array}{l}-0.289^{* *} \\
(-2.18)\end{array}$ & $\begin{array}{l}-0.165 \\
(-1.36)\end{array}$ & $\begin{array}{l}-0.177 \\
(-0.45)\end{array}$ & $\begin{array}{l}-0.568^{* *} \\
(-1.97)\end{array}$ & $\begin{array}{l}-0.785^{* * *} \\
(-6.71)\end{array}$ & -0.143 \\
\hline EM Local - Hedge & $\begin{array}{c}0.085 \\
(1.33)\end{array}$ & $\begin{array}{r}-0.087 \\
(-0.32)\end{array}$ & $\begin{array}{l}-0.123^{* *} \\
(-2.29)\end{array}$ & $\begin{array}{l}-0.062 \\
(-1.45)\end{array}$ & $\begin{array}{l}-0.071 \\
(-1.58)\end{array}$ & $\begin{array}{l}-0.055 \\
(-0.78)\end{array}$ & $\begin{array}{l}-0.020 \\
(-0.13)\end{array}$ & $\begin{array}{c}0.089 \\
(0.46)\end{array}$ & $\begin{array}{l}0.157 \\
(1.34)\end{array}$ & $\begin{array}{l}0.044 \\
(0.47)\end{array}$ & $\begin{array}{l}-0.097 \\
(-1.17)\end{array}$ & -0.055 \\
\hline \multicolumn{13}{|l|}{ Panel B: Frontier Markets } \\
\hline Frontier & $\begin{array}{l}1.321^{* * *} \\
(5.58)\end{array}$ & $\begin{array}{l}1.445^{* * *} \\
(7.74)\end{array}$ & $\begin{array}{l}0.969^{* * *} \\
(3.419\end{array}$ & $\begin{array}{c}0.353 \\
(1.30)\end{array}$ & $\begin{array}{l}0.118 \\
(0.89)\end{array}$ & $\begin{array}{l}-0.117 \\
(-0.89)\end{array}$ & $\begin{array}{l}-0.217 \\
(-0.76)\end{array}$ & $\begin{array}{l}-0.269^{* *} \\
(-2.14)\end{array}$ & $\begin{array}{l}-0.511^{* * *} \\
(-3.04)\end{array}$ & $\begin{array}{l}-1.377^{* * *} \\
(-4.39)\end{array}$ & $\begin{array}{l}-1.373^{* * *} \\
(-5.36)\end{array}$ & -0.037 \\
\hline \multicolumn{13}{|c|}{ Panel C: Developed Markets } \\
\hline France - No Hedge & $\begin{array}{c}0.098 \\
(0.46)\end{array}$ & $\begin{array}{l}-0.025 \\
(-0.12)\end{array}$ & $\begin{array}{l}-0.095 \\
(-0.40)\end{array}$ & $\begin{array}{l}-0.174 \\
(-0.76)\end{array}$ & $\begin{array}{l}-0.136 \\
(-1.18)\end{array}$ & $\begin{array}{l}-0.042 \\
(-0.36)\end{array}$ & $\begin{array}{l}-0.027 \\
(-0.33)\end{array}$ & $\begin{array}{c}0.037 \\
(0.43)\end{array}$ & $\begin{array}{l}-0.044 \\
(-0.44)\end{array}$ & $\begin{array}{l}0.151 \\
(0.04)\end{array}$ & $\begin{array}{l}-0.093 \\
(-0.91)\end{array}$ & -0.065 \\
\hline Germany -No Hedge & $\begin{array}{l}-0.044 \\
(-0.24)\end{array}$ & $\begin{array}{l}-0.028 \\
(-0.15)\end{array}$ & $\begin{array}{l}-0.154 \\
(-0.66)\end{array}$ & $\begin{array}{l}-0.166 \\
(-0.94)\end{array}$ & $\begin{array}{l}-0.101 \\
(-0.93)\end{array}$ & $\begin{array}{l}-0.079 \\
(-0.69)\end{array}$ & $\begin{array}{l}-0.114 \\
(-1.18)\end{array}$ & $\begin{array}{l}-0.028 \\
(-0.43)\end{array}$ & $\begin{array}{l}-0.032 \\
(-0.409\end{array}$ & $\begin{array}{l}0.290 * * * \\
(3.33)\end{array}$ & $\begin{array}{l}-0.191 \\
(-1.51)\end{array}$ & -0.096 \\
\hline Japan - No Hedge & $\begin{array}{c}0.009 \\
(0.09)\end{array}$ & $\begin{array}{c}0.198 \\
(1.23)\end{array}$ & $\begin{array}{c}0.106 \\
(0.93)\end{array}$ & $\begin{array}{c}0.038 \\
(0.27)\end{array}$ & $\begin{array}{l}-0.207 \\
(-1.35)\end{array}$ & $\begin{array}{l}0.036 \\
(0.19)\end{array}$ & $\begin{array}{c}-0.023 \\
(-0.15)\end{array}$ & $\begin{array}{l}-0.014 \\
(-0.03)\end{array}$ & $\begin{array}{l}-0.224 \\
(-0.65)\end{array}$ & $\begin{array}{l}-0.049 \\
(-0.04)\end{array}$ & $\begin{array}{l}-0.030 \\
(-0.03)\end{array}$ & -0.065 \\
\hline UK - No Hedge & $\begin{array}{l}-0.082 \\
(-0.09)\end{array}$ & $\begin{array}{l}-0.225 \\
(-0.85)\end{array}$ & $\begin{array}{l}-0.451 * \\
(-1.65)\end{array}$ & $\begin{array}{l}-0.605^{* * *} \\
(-2.75)\end{array}$ & $\begin{array}{l}-0.588^{* * *} \\
(-3.76)\end{array}$ & $\begin{array}{l}-0.457^{* * *} \\
8-3.64)\end{array}$ & $\begin{array}{l}-0.545^{* * *} \\
(-3.54)\end{array}$ & $\begin{array}{l}-0.481 \\
(-1.55)\end{array}$ & $\begin{array}{l}-0.848^{* *} \\
(-2.18)\end{array}$ & $\begin{array}{l}-1.097^{* * *} \\
(-3.20)\end{array}$ & $\begin{array}{l}-1.257^{* * *} \\
(-5.54)\end{array}$ & -0.525 \\
\hline US - No Hedge & $\begin{array}{l}0.808^{* * *} \\
(5.61)\end{array}$ & $\begin{array}{l}0.485^{* * *} \\
(3.67)\end{array}$ & $\begin{array}{c}0.234 \\
(1.04)\end{array}$ & $\begin{array}{l}-0.009 \\
(-0.09)\end{array}$ & $\begin{array}{l}0.025 \\
(0.38)\end{array}$ & $\begin{array}{l}-0.024 \\
(-0.40)\end{array}$ & $\begin{array}{l}-0.076 \\
(-1.36)\end{array}$ & $\begin{array}{l}-0.119 \\
(-1.33)\end{array}$ & $\begin{array}{l}-0.102 \\
(-0.97)\end{array}$ & $\begin{array}{l}-0.105 \\
(-1.24)\end{array}$ & $\begin{array}{l}-0.029 \\
(-0.37)\end{array}$ & 0.004 \\
\hline France - Hedged & $\begin{array}{l}-0.609^{* * * *} \\
(-3.31)\end{array}$ & $\begin{array}{l}-0.528^{* *} \\
(-2.04)\end{array}$ & $\begin{array}{l}-0.069 \\
(-0.32)\end{array}$ & $\begin{array}{c}0.099 \\
(1.41)\end{array}$ & $\begin{array}{l}0.001 \\
(0.02)\end{array}$ & $\begin{array}{l}0.115 \\
(1.50)\end{array}$ & $\begin{array}{c}0.116 \\
(1.49)\end{array}$ & $\begin{array}{c}0.062 \\
(0.96)\end{array}$ & $\begin{array}{l}0.052 \\
(0.72)\end{array}$ & $\begin{array}{l}-0.135 \\
(-0.94)\end{array}$ & $\begin{array}{l}-0.536^{* *} \\
(-2.59)\end{array}$ & 0.049 \\
\hline Germany - Hedged & $\begin{array}{l}-0.691^{* * *} \\
(-6.78)\end{array}$ & $\begin{array}{l}-0.425 \\
(-1.37)\end{array}$ & $\begin{array}{l}-0.159 \\
(-0.76)\end{array}$ & $\begin{array}{c}0.049 \\
(0.50)\end{array}$ & $\begin{array}{l}-0.031 \\
(-0.51)\end{array}$ & $\begin{array}{l}0.070 \\
(1.00)\end{array}$ & $\begin{array}{c}0.108 \\
(1.32)\end{array}$ & $\begin{array}{c}0.043 \\
(0.72)\end{array}$ & $\begin{array}{l}-0.032 \\
(-0.50)\end{array}$ & $\begin{array}{l}-0.148^{*} \\
(-1.94)\end{array}$ & $\begin{array}{l}-0.020 \\
(-0.23)\end{array}$ & 0.019 \\
\hline Japan - Hedged & $\begin{array}{r}0.187 \\
(0.90)\end{array}$ & $\begin{array}{c}0.092 \\
(0.15)\end{array}$ & $\begin{array}{l}-0.060 \\
(-0.29)\end{array}$ & $\begin{array}{l}-0.062 \\
(-1.41)\end{array}$ & $\begin{array}{l}0.005 \\
(0.09)\end{array}$ & $\begin{array}{l}0.103^{* * * *} \\
(3.00)\end{array}$ & $\begin{array}{c}0.043 \\
(1.12)\end{array}$ & $\begin{array}{c}0.035 \\
(0.90)\end{array}$ & $\begin{array}{l}-0.030 \\
(-0.83)\end{array}$ & $\begin{array}{l}-0.070 \\
(-0.41)\end{array}$ & $\begin{array}{l}0.023 \\
(0.23)\end{array}$ & 0.032 \\
\hline UK - Hedged & $\begin{array}{l}-0.208^{*} \\
(-1.67)\end{array}$ & $\begin{array}{l}-0.273 \\
(-1.50)\end{array}$ & $\begin{array}{r}0.127 \\
(0.72)\end{array}$ & $\begin{array}{l}-0.093 \\
(-0.19)\end{array}$ & $\begin{array}{l}0.058 \\
(0.45)\end{array}$ & $\begin{array}{l}0.063 \\
(0.68)\end{array}$ & $\begin{array}{l}-0.075 \\
(-0.54)\end{array}$ & $\begin{array}{l}-0.148 \\
(-1.03)\end{array}$ & $\begin{array}{l}-0.393 \\
(-2.30)\end{array}$ & $\begin{array}{l}-0.141 \\
(-1.21)\end{array}$ & $\begin{array}{l}-0.571 \\
(-1.14)\end{array}$ & -0.018 \\
\hline
\end{tabular}

This table reports the quantile regression coefficient estimates of $\gamma_{1}$ (change of the dependence during the global financial crisis period) according to the model defined by Equation (1). T-statistics are in parentheses. The last column shows change in degree of dependence. 
Table 4

Structure and degree of the dependence (change during the Eurozone debt crisis period).

\begin{tabular}{|c|c|c|c|c|c|c|c|c|c|c|c|c|}
\hline Quantile & 1 & 2 & 5 & 10 & 25 & 50 & 75 & 90 & 95 & 98 & 99 & Degree \\
\hline \multicolumn{13}{|c|}{ Panel A: Emerging Markets } \\
\hline EMBI + & $\begin{array}{l}0.741^{* * * *} \\
(2.75)\end{array}$ & $\begin{array}{l}0.793^{* *} \\
(2.59)\end{array}$ & $\begin{array}{c}0.312 \\
(0.80)\end{array}$ & $\begin{array}{r}0.001 \\
(0.01)\end{array}$ & $\begin{array}{l}-0.081 \\
(-0.49)\end{array}$ & $\begin{array}{l}-0.019 \\
(-0.16)\end{array}$ & $\begin{array}{l}-0.148 \\
(-1.26)\end{array}$ & $\begin{array}{l}-0.476^{* * *} \\
(-3.03)\end{array}$ & $\begin{array}{l}-0.792^{* * *} \\
(-4.13)\end{array}$ & $\begin{array}{l}-1.159^{* * *} \\
(-5.23)\end{array}$ & $\begin{array}{l}-1.727^{* * *} \\
(-9.37)\end{array}$ & -0.115 \\
\hline EM Local - No Hedge & $\begin{array}{l}0.857^{* * *} \\
(3.87)\end{array}$ & $\begin{array}{l}0.969^{* * *} \\
(4.49)\end{array}$ & $\begin{array}{c}0.272 \\
(0.76)\end{array}$ & $\begin{array}{c}0.096 \\
(0.33)\end{array}$ & $\begin{array}{l}-0.101 \\
(-0.90)\end{array}$ & $\begin{array}{l}-0.120 \\
(-1.07)\end{array}$ & $\begin{array}{l}-0.150 \\
(-1.14)\end{array}$ & $\begin{array}{l}-0.077 \\
(-0.38)\end{array}$ & $\begin{array}{l}0.211 \\
(0.78)\end{array}$ & $\begin{array}{l}0.555^{* * *} \\
(2.69)\end{array}$ & $\begin{array}{l}0.288 \\
(0.99)\end{array}$ & -0.015 \\
\hline EM Local - Hedge & $\begin{array}{c}0.169 \\
(0.42)\end{array}$ & $\begin{array}{l}-0.013 \\
(-0.05)\end{array}$ & $\begin{array}{l}-0.147^{* * *} \\
(-2.17)\end{array}$ & $\begin{array}{l}-0.045 \\
(-0.58)\end{array}$ & $\begin{array}{l}0.044 \\
(1.42)\end{array}$ & $\begin{array}{l}-0.029 \\
(-1.05)\end{array}$ & $\begin{array}{l}-0.064 \\
(-1.47)\end{array}$ & $\begin{array}{l}-0.039 \\
(-0.25)\end{array}$ & $\begin{array}{l}0.128 \\
(0.48)\end{array}$ & $\begin{array}{l}-0.050 \\
(-0.14)\end{array}$ & $\begin{array}{l}-0.281 \\
(-0.89)\end{array}$ & -0.027 \\
\hline \multicolumn{13}{|l|}{ Panel B: Frontier Markets } \\
\hline Frontier & $\begin{array}{l}-0.733 \\
(-0.08)\end{array}$ & $\begin{array}{l}-0.176 \\
(-0.02)\end{array}$ & $\begin{array}{l}-0.139 \\
(-0.05)\end{array}$ & $\begin{array}{c}0.009 \\
(0.02)\end{array}$ & $\begin{array}{l}0.216 \\
(1.319\end{array}$ & $\begin{array}{l}0.125 \\
(1.04)\end{array}$ & $\begin{array}{l}0.148 \\
(1.06)\end{array}$ & $\begin{array}{l}-0.028 \\
(-0.18)\end{array}$ & $\begin{array}{l}-0.341^{*} \\
(-1.87)\end{array}$ & $\begin{array}{l}-0.669^{*} \\
(-1.87)\end{array}$ & $\begin{array}{l}-0.737^{\text {*** }} \\
(-2.45)\end{array}$ & 0.047 \\
\hline \multicolumn{13}{|c|}{ Panel C: Developed Markets } \\
\hline France - No Hedge & $\begin{array}{l}0.399^{* * * *} \\
(2.88)\end{array}$ & $\begin{array}{l}0.268^{*} \\
(1.71)\end{array}$ & $\begin{array}{c}0.142 \\
(1.07)\end{array}$ & $\begin{array}{c}0.214 \\
(1.49)\end{array}$ & $\begin{array}{l}0.135 \\
(1.34)\end{array}$ & $\begin{array}{l}0.130 \\
(1.49)\end{array}$ & $\begin{array}{l}0.302^{* * *} \\
(3.32)\end{array}$ & $\begin{array}{l}0.125 \\
(1.28)\end{array}$ & $\begin{array}{l}0.183 \\
(1.32)\end{array}$ & $\begin{array}{l}0.441 \\
(1.44)\end{array}$ & $\begin{array}{l}0.749^{* * * *} \\
(3.02)\end{array}$ & 0.195 \\
\hline Germany -No Hedge & $\begin{array}{l}0.307^{* *} \\
(2.22)\end{array}$ & $\begin{array}{c}0.221 \\
(1.44)\end{array}$ & $\begin{array}{c}0.054 \\
(0.39)\end{array}$ & $\begin{array}{l}-0.075 \\
(-0.80)\end{array}$ & $\begin{array}{l}0.036 \\
(0.35)\end{array}$ & $\begin{array}{l}0.117 \\
(1.25)\end{array}$ & $\begin{array}{l}0.164 \\
(1.63)\end{array}$ & $\begin{array}{l}0.155 \\
(1.18)\end{array}$ & $\begin{array}{l}0.260 \\
(1.22)\end{array}$ & $\begin{array}{l}0.406^{*} \\
(1.80)\end{array}$ & $\begin{array}{l}0.471 \\
(2.55)\end{array}$ & 0.109 \\
\hline Japan - No Hedge & $\begin{array}{c}0.145 \\
(0.34)\end{array}$ & $\begin{array}{c}0.415 \\
(0.80)\end{array}$ & $\begin{array}{r}0.107 \\
(0.31)\end{array}$ & $\begin{array}{c}0.002 \\
(0.01)\end{array}$ & $\begin{array}{l}0.062 \\
(0.34)\end{array}$ & $\begin{array}{l}0.111 \\
(0.80)\end{array}$ & $\begin{array}{l}-0.089 \\
(-0.66)\end{array}$ & $\begin{array}{l}-0.064 \\
(-0.36)\end{array}$ & $\begin{array}{l}-0.403 \\
(-1.25)\end{array}$ & $\begin{array}{l}-0.516^{*} \\
(-1.81)\end{array}$ & $\begin{array}{l}-0.757^{\text {*k* }} \\
(-3.79)\end{array}$ & 0.053 \\
\hline UK - No Hedge & $\begin{array}{c}-0.138 \\
(-0.19)\end{array}$ & $\begin{array}{l}-0.541 \\
(-1.08)\end{array}$ & $\begin{array}{l}-0.495 \\
(-1.85)\end{array}$ & $\begin{array}{l}-0.022 \\
(-0.07)\end{array}$ & $\begin{array}{l}-0.207 \\
(-1.04)\end{array}$ & $\begin{array}{l}-0.278^{* *} \\
(-2.54)\end{array}$ & $\begin{array}{l}-0.173 \\
(-1.04)\end{array}$ & $\begin{array}{l}-0.210 \\
(-1.02)\end{array}$ & $\begin{array}{l}-0.254 \\
(-0.95)\end{array}$ & $\begin{array}{l}0.187 \\
(0.26)\end{array}$ & $\begin{array}{l}0.728 \\
(1.33)\end{array}$ & -0.206 \\
\hline US - No Hedge & $\begin{array}{l}0.632^{* * *} \\
(4.67)\end{array}$ & $\begin{array}{l}0.292^{* *} \\
(2.27)\end{array}$ & $\begin{array}{r}0.014 \\
(0.109\end{array}$ & $\begin{array}{l}-0.015 \\
(-0.27)\end{array}$ & $\begin{array}{l}-0.158^{* *} \\
(-2.31)\end{array}$ & $\begin{array}{l}-0.179^{* * *} \\
(-2.61)\end{array}$ & $\begin{array}{l}-0.175^{* * *} \\
(-2.60)\end{array}$ & $\begin{array}{l}-0.166^{*} \\
(-1.86)\end{array}$ & $\begin{array}{l}-0.209^{* * *} \\
(-2.81)\end{array}$ & $\begin{array}{l}-0.298^{* * *} \\
(-3.16)\end{array}$ & $\begin{array}{l}-0.264^{* * *} \\
(-3.21)\end{array}$ & -0.147 \\
\hline France - Hedged & $\begin{array}{r}0.051 \\
(0.76)\end{array}$ & $\begin{array}{c}0.094 \\
(0.64)\end{array}$ & $\begin{array}{l}-0.176 \\
(-1.09)\end{array}$ & $\begin{array}{r}-0.197 \\
(-1.63)\end{array}$ & $\begin{array}{l}-0.102 \\
(-1.16)\end{array}$ & $\begin{array}{l}-0.053 \\
(-0.63)\end{array}$ & $\begin{array}{l}0.008 \\
(0.08)\end{array}$ & $\begin{array}{l}0.063 \\
(0.74)\end{array}$ & $\begin{array}{l}0.101 \\
(0.78)\end{array}$ & $\begin{array}{l}-0.075 \\
(-0.36)\end{array}$ & $\begin{array}{l}0.378^{* *} \\
(2.04)\end{array}$ & $-0.03 \mathrm{~s}$ \\
\hline Germany - Hedged & $\begin{array}{l}-0.255 \\
(-1.57)\end{array}$ & $\begin{array}{l}-0.285 \\
(-1.34)\end{array}$ & $\begin{array}{l}-0.449 \\
(-3.06)\end{array}$ & $\begin{array}{c}-0.199 \\
(-0.86)\end{array}$ & $\begin{array}{l}-0.175^{* *} \\
(-2.50)\end{array}$ & $\begin{array}{l}-0.159^{*} \\
(-1.84)\end{array}$ & $\begin{array}{l}-0.014 \\
(-0.13)\end{array}$ & $\begin{array}{l}-0.079 \\
(-1.29)\end{array}$ & $\begin{array}{l}-0.079 \\
(-0.92)\end{array}$ & $\begin{array}{l}0.138 \\
(0.70)\end{array}$ & $\begin{array}{l}0.112 \\
(0.44)\end{array}$ & -0.126 \\
\hline Japan - Hedged & $\begin{array}{r}0.146 \\
(0.56)\end{array}$ & $\begin{array}{c}0.038 \\
(0.06)\end{array}$ & $\begin{array}{l}-0.155^{* *} \\
(-2.39)\end{array}$ & $\begin{array}{c}-0.023 \\
(-0.49)\end{array}$ & $\begin{array}{l}0.041 \\
(1.29)\end{array}$ & $\begin{array}{l}0.014 \\
(0.38)\end{array}$ & $\begin{array}{l}0.035 \\
(1.04)\end{array}$ & $\begin{array}{l}-0.031 \\
(-0.69)\end{array}$ & $\begin{array}{l}-0.133^{* * *} \\
(-2.70)\end{array}$ & $\begin{array}{l}-0.293^{* *} \\
(-2.37)\end{array}$ & $\begin{array}{l}-0.320^{* * *} \\
(-3.10)\end{array}$ & -0.002 \\
\hline UK - Hedged & $\begin{array}{c}0.352 \\
(1.33)\end{array}$ & $\begin{array}{c}0.050 \\
(0.09)\end{array}$ & $\begin{array}{c}0.066 \\
(0.28)\end{array}$ & $\begin{array}{l}-0.252^{*} \\
(-1.89)\end{array}$ & $\begin{array}{l}-0.058 \\
(-0.49)\end{array}$ & $\begin{array}{l}-0.073 \\
(-0.76)\end{array}$ & $\begin{array}{l}-0.142 \\
(-1.03)\end{array}$ & $\begin{array}{l}-0.288^{* *} \\
(-2.21)\end{array}$ & $\begin{array}{l}-0.459^{* * *} \\
(-2.76)\end{array}$ & $\begin{array}{l}-0.157 \\
(-0.55)\end{array}$ & $\begin{array}{l}-0.437 \\
(-1.22)\end{array}$ & -0.118 \\
\hline
\end{tabular}

This table reports the quantile regression coefficient estimates of $\gamma_{2}$ (change of the dependence during the Eurozone debt crisis period) according to the model defined by Equation (1). T-statistics are in parentheses. The last column shows change in degree of dependence. 


\section{Panel A: Dependence in local-currency emerging bond markets}
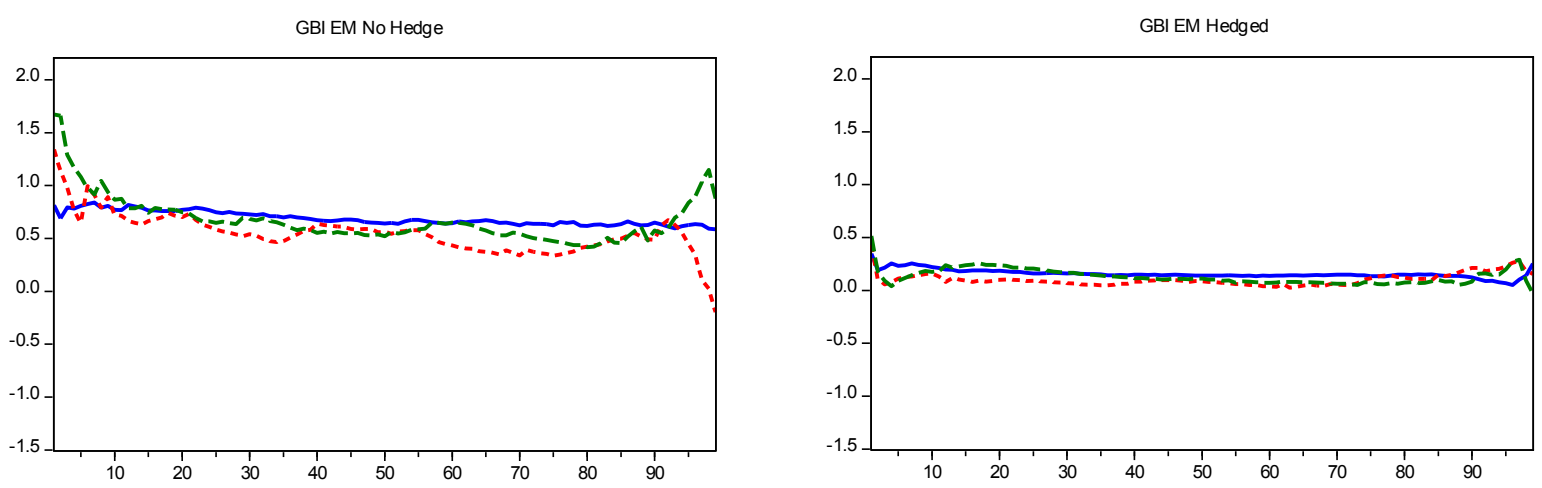

Panel B: Dependence in USD-denominated emerging and frontier bond markets
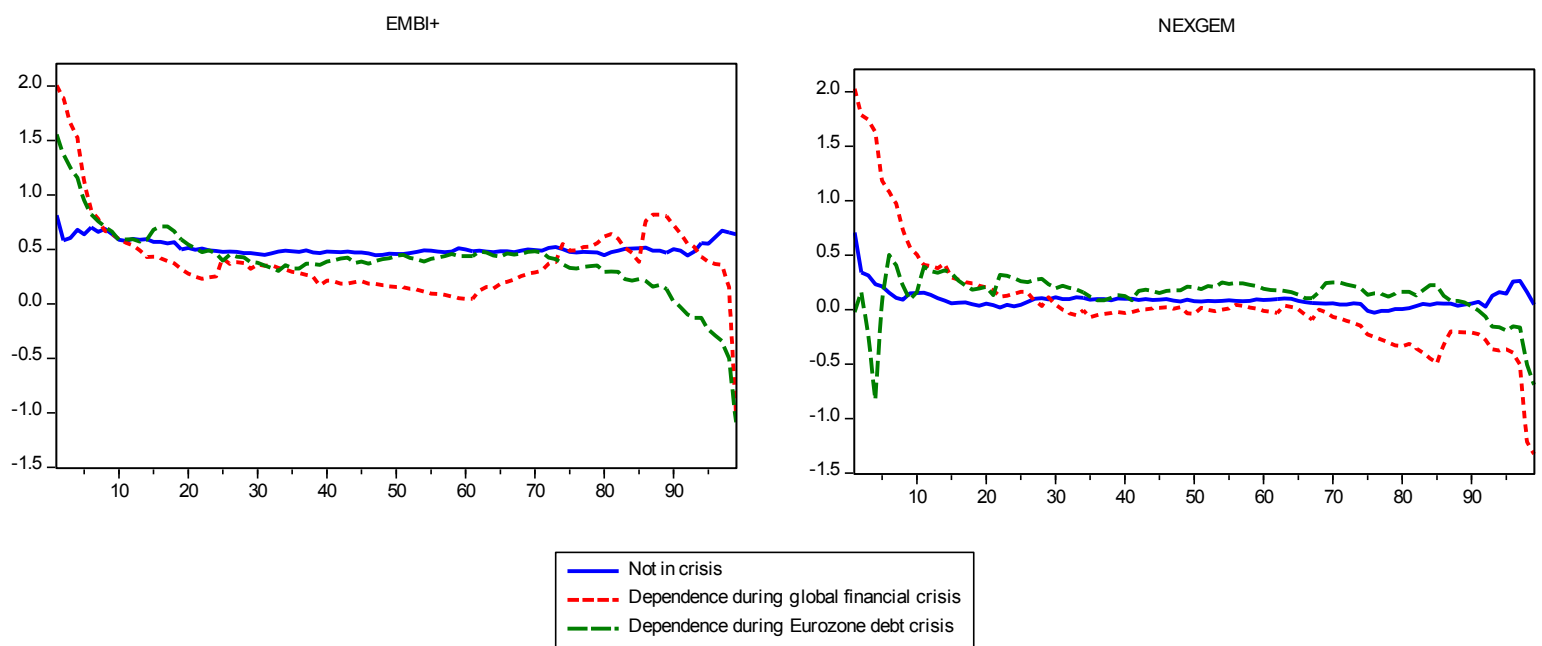

Fig. 2. Structure of dependence for emerging and frontier markets. This figure shows dependence structure of emerging and frontier markets bond returns on the returns of global bond market portfolio. Panel A refers to dependence in local-currency for emerging bond markets (graph on left side shows the case of unhedged returns and graph on right side shows returns hedged to US dollar using currency derivatives). Panel B refers to returns dependence in USD-denominated emerging and frontier bond markets. The blue line represents the normal period dependence (given by coefficient estimates $\beta$ ). The red line represents aggregate dependence in the global financial crisis period (sum of coefficient estimates $\beta$ and $\gamma_{1}$ ), and the green line represents aggregate dependence in the Eurozone debt crisis period (sum of coefficient estimates $\beta$ and $\gamma_{2}$ ). Quantiles are represented on the horizontal axis and values of estimated coefficients from quantile regression are represented on the vertical axis. (For interpretation of the references to colour in this figure legend, the reader is referred to the web version of this article.)

degree of dependence contingent on whether emerging markets local currency returns are hedged or unhedged. The further evidence suggests that the global financial crisis and the Eurozone debt crisis led to a negative change in the degree of dependence for both hedged and unhedged returns. At the same time, the magnitude of change during the global financial crisis $\left(\gamma_{1}\right)$ is higher than during the Eurozone debt crisis $\left(\gamma_{2}\right)$. More substantial effects during the global financial crisis can be explained by the fact that this crisis originated from equity markets, and investors perceive emerging markets bonds as "equity-like assets" (Piljak, 2013). On the contrary, the Eurozone debt crisis emanated from uncertainty in debt markets, and its most significant and immediate impact was witnessed in European developed markets (see, Gómez-Puig and Sosvilla-Rivero, 2014).

\subsection{Dependence in USD-denominated emerging and frontier bond markets}

Emerging markets USD-denominated returns (shown in Fig. 2, Panel B) exhibit a similar dependence pattern to emerging markets local currency unhedged returns in the global financial crisis, albeit with a larger magnitude. This finding is in line with Agur et al. (2019), who report that emerging market USD-denominated debt markets are more internationally integrated than emerging markets local currency bond markets. Furthermore, credit spreads for emerging market debt move in line with emerging market currencies. During the Eurozone debt crisis, the dependence pattern for emerging USD-denominated debt and unhedged local currency debt is the 

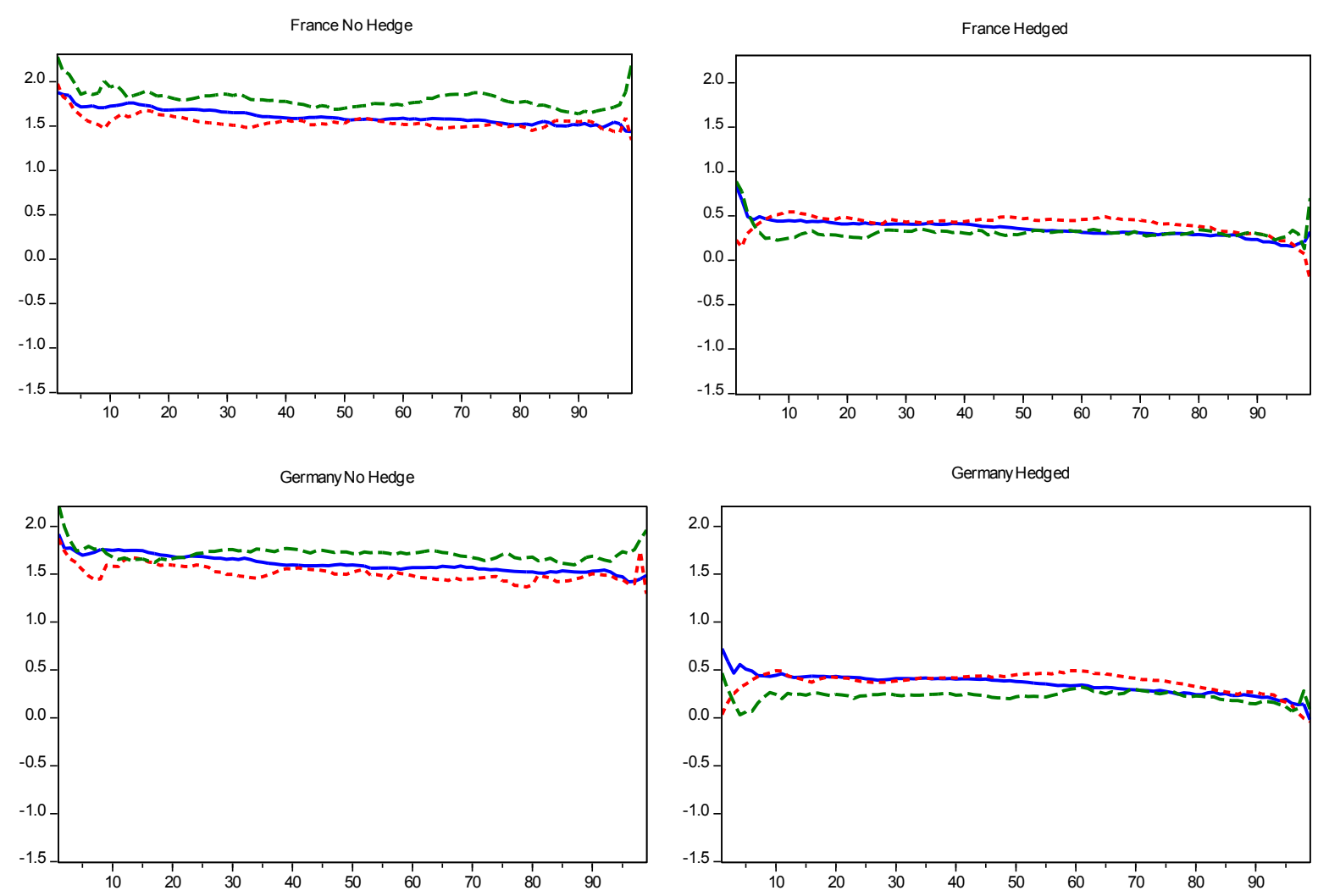

Japan No Hedge
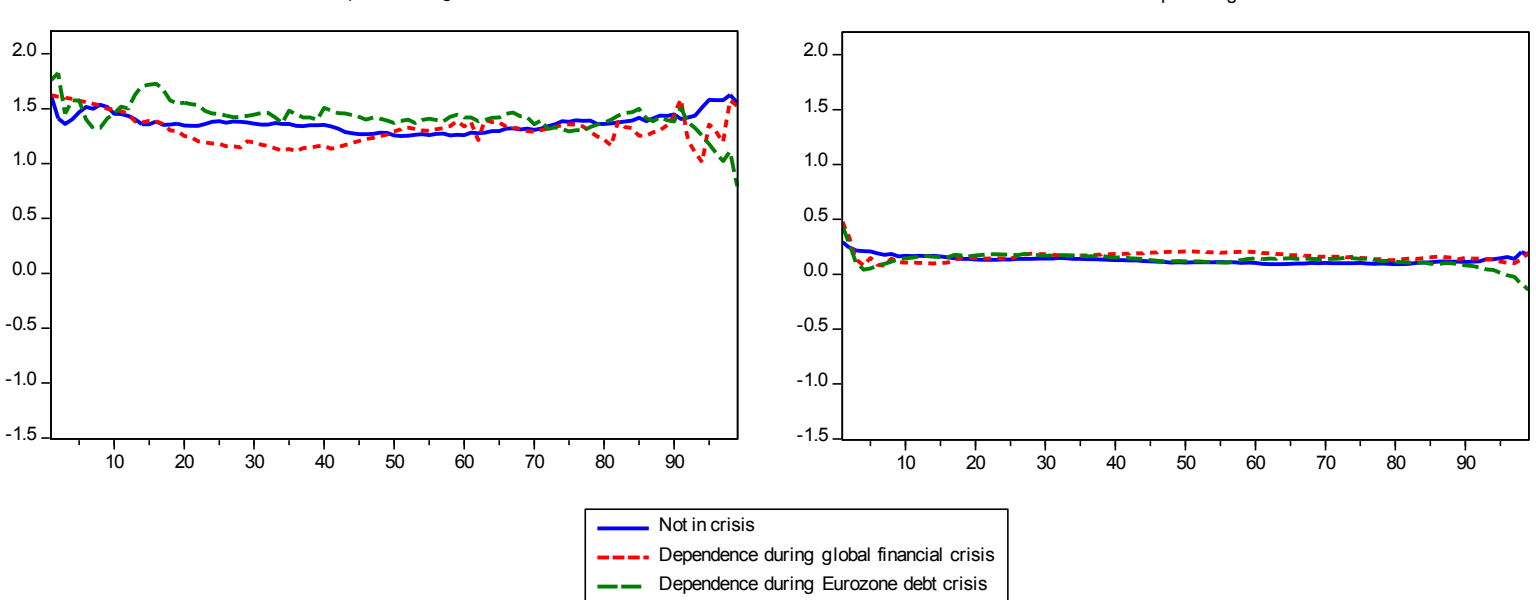

Fig. 3. Structure of dependence for developed markets. This figure shows dependence structure of developed markets bond returns on the returns of global bond market portfolio. The graphs on the left side refer to the unhedged returns, while graphs on the right side refer to returns hedged to US dollar using currency derivatives. Description of lines and axes is the same as in Fig. 2.

same in the left tail but different in the right tail. In particular, dependence in the right tail is increasing for unhedged local currency sample, but decreasing for emerging USD-denominated sample. Apparently, this pattern indicates that the currency denomination of emerging markets bonds plays a vital role in reacting to the Eurozone debt crisis, which was also the crisis of the Euro, a common Eurozone currency. In a broader context, our results are corresponding to findings of Burger et al. (2018), stating that currency denomination is a very important aspect of international bond portfolio management. Looking deeper at the regional level ${ }^{12}$, it appears

$\overline{12}$ See Appendix B (Fig. B.1) for graphical representation of the structure and Table B.1 for degree of dependence. 

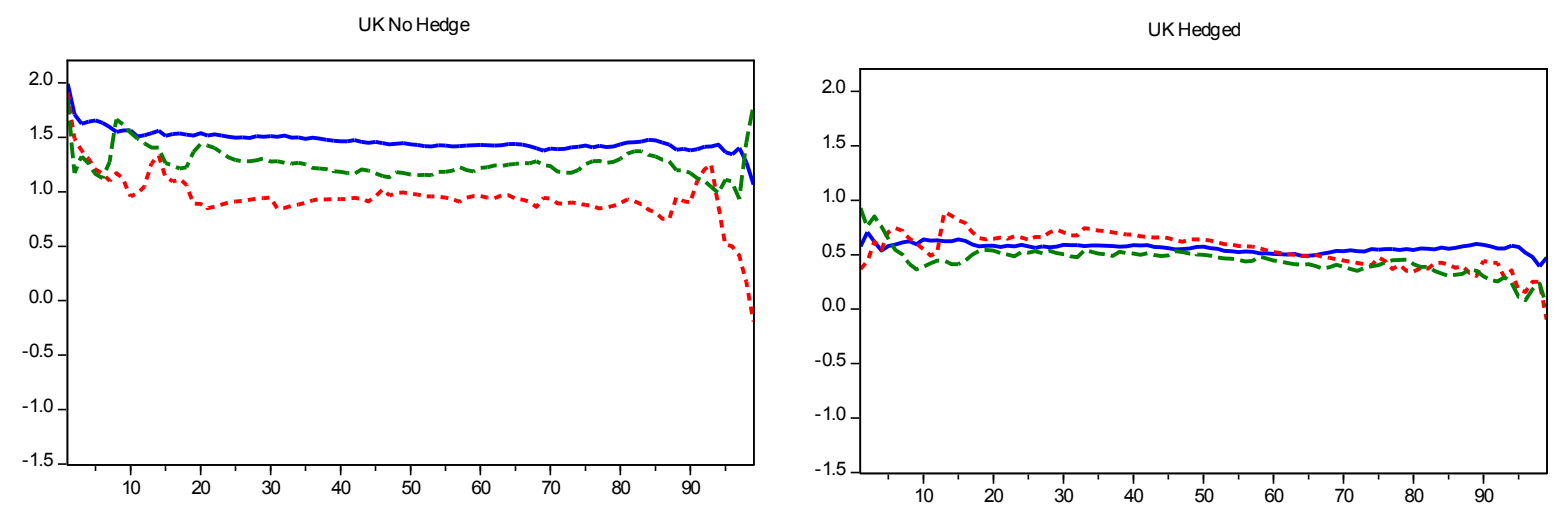

USA No Hedge

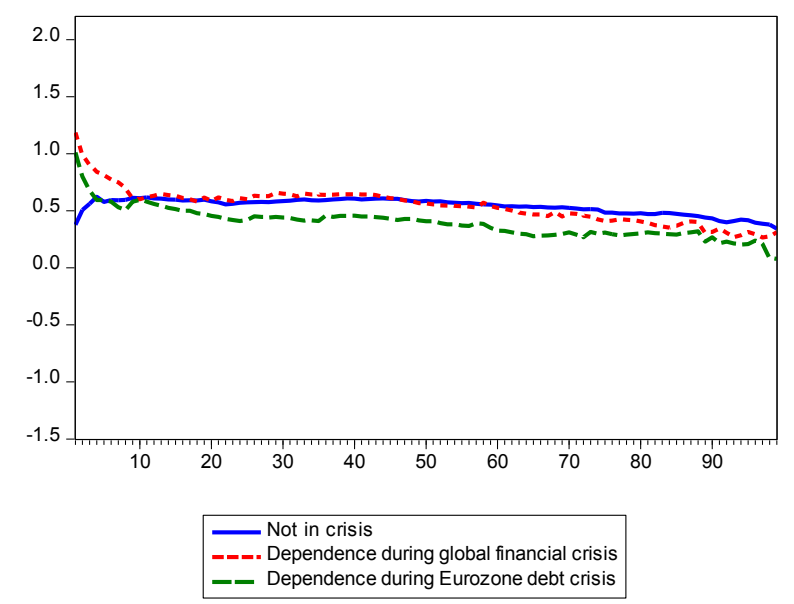

Fig. 3. (continued).

that dependence patterns of Asian, European, and Latin American regions resemble the pattern of aggregate emerging markets level. At the same time, the African region shows a slightly different pattern in the right tail during the global financial crisis (coefficient values are still positive even in the most extreme quantiles in the right tail). This indicates that the degree of bond market integration of emerging markets in the African region is different from the integration level of other regions. The analysis on country level ${ }^{13}$ reveals that all countries have very similar dependence pattern (high statistical significance across quantiles) to aggregate level. During the global financial crisis, the majority of countries follow the same pattern regarding the change of dependence (high statistical significance in extreme left and right tail quantiles), with the exception of South Africa (both tails) and Philippines (left tail).

The dependence of frontier markets bond return relative to the global bond market (shown in Fig. 3, Panel B) is similar to one observed by emerging markets USD-denominated return. The only difference arises during the Eurozone debt crisis in the left tail. In that instance, the dependence for the emerging markets USD-denominated index is approximately 1.5 in the left tail, vs. near zero for frontier markets. This observed dependence behavior in the left tail cannot be captured with the linear dependence structure approach implemented in Piljak and Swinkels (2017a). Our ability to report this type of results stems from the application of the quantile regression approach. To offer further insights into the dependence patterns of frontier markets, we conduct regional and country analysis. ${ }^{14}$ The pattern observed in every individual country is not the same as the pattern on the aggregate level. In particular, in precrisis period there is a statistical significance only in left tail (quantiles 1-10) on the aggregate level, while in certain countries (Egypt and Ecuador) there is also statistically significant dependence in right tail (quantiles 90-99). Furthermore, a set of results derived from observation of regional bond markets reveals that the Latin American region has a much larger magnitude of dependence in both left and right tail than other regions during two examined crises events. In particular, during the Eurozone debt crisis, Dominican Republic and Ecuador have a significant change of dependence in the left tail, which is in contrast to the aggregate frontier markets level where there is no significant change. In summary, results of the regional analysis are consistent with literature emphasizing that emerging and frontier bond markets are not homogenous groups of markets and hence that they have a different vulnerability to crises (Christopher

\footnotetext{
13 See Appendix C (Tables C.1, C.2, and C.3) for country-specific detailed results.

14 See Appendix B (Fig. B.2) for graphical representation and Appendix C (Tables C.1, C.2, and C.3) for country-specific detailed results.
} 
Table 5

Significance tests of inter-quantile differences.

\begin{tabular}{|c|c|c|c|c|c|c|}
\hline & $\mathrm{q} 0.01=\mathrm{q} 0.50$ & $\mathrm{q} 0.02=\mathrm{q} 0.50$ & $\mathrm{q} 0.05=\mathrm{q} 0.50$ & $\mathrm{q} 0.95=\mathrm{q} 0.50$ & $\mathrm{q} 0.98=\mathrm{q} 0.50$ & $\mathrm{q} 0.99=\mathrm{q} 0.50$ \\
\hline \multicolumn{7}{|c|}{ Panel A: Emerging Markets } \\
\hline \multicolumn{7}{|c|}{ EMBI +} \\
\hline \multirow[t]{2}{*}{ Not in crisis } & $0.36^{* *}$ & 0.13 & $0.18^{*}$ & -0.10 & -0.20 & -0.18 \\
\hline & $(0.14)$ & $(0.09)$ & $(0.10)$ & $(0.12)$ & $(0.13)$ & $(0.11)$ \\
\hline \multirow[t]{2}{*}{ Financial crisis } & $1.49^{* * * *}$ & $1.61^{* * *}$ & 0.79 & -0.13 & 0.20 & $1.35^{* * *}$ \\
\hline & $(0.17)$ & $(0.16)$ & $(0.54)$ & $(0.22)$ & $(0.34)$ & $(0.19)$ \\
\hline \multirow[t]{2}{*}{ Debt crisis } & $0.76^{* * *}$ & $0.81^{* * *}$ & 0.33 & $0.77^{* * * *}$ & $1.14^{* * *}$ & $1.71^{* * *}$ \\
\hline & $(0.29)$ & $(0.31)$ & $(0.39)$ & $(0.21)$ & $(0.24)$ & $(0.21)$ \\
\hline \multicolumn{7}{|c|}{ GBI EM Local Currency } \\
\hline \multirow[t]{2}{*}{ Not in crisis } & 0.17 & 0.05 & $0.17 * *$ & 0.01 & 0.05 & 0.05 \\
\hline & $(0.16)$ & $(0.11)$ & $(0.08)$ & $(0.11)$ & $(0.12)$ & $(0.11)$ \\
\hline \multirow[t]{2}{*}{ Financial crisis } & $0.60^{*}$ & 0.53 & -0.08 & 0.10 & 0.49 & $0.71^{* * *}$ \\
\hline & $(0.31)$ & $(0.62)$ & (1.19) & $(0.39)$ & $(0.30)$ & $(0.17)$ \\
\hline \multirow[t]{2}{*}{ Debt crisis } & $0.98^{* * *}$ & $1.09 * * *$ & 0.39 & -0.33 & $-0.68^{* * *}$ & -0.41 \\
\hline & $(0.24)$ & $(0.23)$ & $(0.35)$ & $(0.27)$ & $(0.22)$ & $(0.30)$ \\
\hline \multicolumn{7}{|c|}{ GBI EM Local Currency - Hedged } \\
\hline \multirow[t]{2}{*}{ Not in crisis } & $0.21^{* *}$ & 0.05 & $0.09^{* * * *}$ & $0.07^{* *}$ & 0.00 & -0.11 \\
\hline & $(0.10)$ & $(0.03)$ & $(0.03)$ & $(0.03)$ & $(0.08)$ & $(0.08)$ \\
\hline \multirow[t]{2}{*}{ Financial crisis } & 0.14 & -0.03 & -0.07 & $-0.21^{*}$ & -0.10 & 0.04 \\
\hline & $(0.09)$ & $(0.27)$ & $(0.08)$ & $(0.12)$ & $(0.11)$ & $(0.11)$ \\
\hline Debt crisis & 0.20 & 0.02 & $-0.12^{*}$ & -0.16 & 0.02 & 0.25 \\
\hline & $(0.40)$ & $(0.29)$ & $(0.07)$ & $(0.26)$ & $(0.35)$ & $(0.31)$ \\
\hline Panel B: Fronti & & & & & & \\
\hline NEXGEM & & & & & & \\
\hline Not in crisis & $0.63^{* *}$ & $0.26^{*}$ & 0.14 & -0.07 & -0.08 & 0.03 \\
\hline & $(0.27)$ & $(0.15)$ & $(0.10)$ & $(0.12)$ & $(0.12)$ & $(0.14)$ \\
\hline Financial crisis & $1.44^{* * *}$ & $1.56^{* * *}$ & $1.09^{* * *}$ & $0.39 * *$ & $1.26^{* * *}$ & $1.26^{* * *}$ \\
\hline & $(0.26)$ & $(0.21)$ & $(0.29)$ & $(0.19)$ & $(0.33)$ & $(0.28)$ \\
\hline Debt crisis & -0.86 & -0.30 & -0.26 & $0.47 * *$ & $0.79 * *$ & $0.86^{* * *}$ \\
\hline & $(9.56)$ & $(7.41)$ & $(2.61)$ & $(0.20)$ & $(0.36)$ & $(0.31)$ \\
\hline Panel C: Devel & & & & & & \\
\hline France & & & & & & \\
\hline Not in crisis & $0.31^{* * * *}$ & $0.28^{* * * *}$ & $0.14 * *$ & 0.06 & 0.13 & 0.14 \\
\hline & $(0.11)$ & $(0.10)$ & $(0.06)$ & $(0.08)$ & $(0.10)$ & $(0.12)$ \\
\hline Financial crisis & 0.14 & 0.02 & -0.05 & 0.00 & -0.19 & 0.05 \\
\hline & $(0.23)$ & $(0.23)$ & $(0.24)$ & $(0.14)$ & $(3.50)$ & $(0.15)$ \\
\hline Debt crisis & $0.27^{*}$ & 0.14 & 0.01 & -0.05 & -0.31 & $-0.62^{* *}$ \\
\hline & $(0.16)$ & $(0.17)$ & $(0.14)$ & $(0.15)$ & $(0.31)$ & $(0.26)$ \\
\hline Germany & & & & & & \\
\hline Not in crisis & $0.32^{* * * *}$ & $0.17^{*}$ & $0.10^{*}$ & 0.12 & $0.15^{* * *}$ & 0.11 \\
\hline & $(0.11)$ & $(0.09)$ & $(0.06)$ & $(0.09)$ & $(0.05)$ & $(0.06)$ \\
\hline Financial crisis & 0.03 & 0.05 & -0.07 & -0.05 & $-0.37^{* * *}$ & $0.11^{*}$ \\
\hline & $(0.20)$ & $(0.21)$ & $(0.24)$ & $(0.13)$ & $(0.14)$ & $(0.16)$ \\
\hline Debt crisis & 0.19 & 0.10 & -0.06 & -0.14 & -0.29 & $-0.35^{*}$ \\
\hline & $(0.16)$ & $(0.17)$ & $(0.15)$ & $(0.21)$ & $(0.23)$ & $(0.20)$ \\
\hline Japan & & & & & & \\
\hline Not in crisis & $0.36^{* * * *}$ & 0.15 & $0.21^{* *}$ & $-0.32^{* * *}$ & $-0.37^{* * * *}$ & $-0.30^{* * *}$ \\
\hline & $(0.12)$ & $(0.15)$ & $(0.10)$ & $(0.09)$ & $(0.09)$ & $(0.09)$ \\
\hline Financial crisis & -0.03 & 0.16 & 0.07 & 0.26 & 0.09 & 0.07 \\
\hline & $(0.22)$ & $(0.24)$ & $(0.21)$ & $(0.36)$ & $(1.12)$ & $(0.96)$ \\
\hline Debt crisis & 0.03 & 0.30 & 0.00 & 0.51 & $0.63 * *$ & $0.87^{* * * *}$ \\
\hline & $(0.44)$ & $(0.52)$ & $(0.35)$ & $(0.32)$ & $(0.30)$ & $(0.23)$ \\
\hline Table 5 (Cont.) & ter-quantile diff & ices & & & & \\
\hline & $\mathrm{q} 0.01=\mathrm{q} 0.50$ & $\mathrm{q} 0.02=\mathrm{q} 0.50$ & $\mathrm{q} 0.05=\mathrm{q} 0.50$ & $\mathrm{q} 0.95=\mathrm{q} 0.50$ & $\mathrm{q} 0.98=\mathrm{q} 0.50$ & $\mathrm{q} 0.99=\mathrm{q} 0.50$ \\
\hline UK & & & & & & \\
\hline Not in crisis & $0.56^{*}$ & $0.28^{* *}$ & $0.22^{* * *}$ & 0.07 & 0.17 & $0.37^{* *}$ \\
\hline & $(0.30)$ & $(0.13)$ & $(0.09)$ & $(0.10)$ & $(0.15)$ & $(0.14)$ \\
\hline Financial crisis & 0.37 & 0.23 & 0.01 & 0.39 & $0.64^{*}$ & $0.80^{* * *}$ \\
\hline & $(0.95)$ & $(0.28)$ & $(0.27)$ & $(0.38)$ & $(0.35)$ & $(0.25)$ \\
\hline Debt crisis & 0.14 & -0.26 & -0.22 & -0.02 & -0.46 & $-1.01 *$ \\
\hline & $(0.74)$ & $(0.50)$ & $(0.27)$ & $(0.26)$ & $(0.73)$ & $(0.55)$ \\
\hline US & & & & & & \\
\hline Not in crisis & $-0.21^{* * * *}$ & -0.08 & -0.01 & $0.17^{* * * *}$ & $0.21^{* * *}$ & $0.24^{* * * *}$ \\
\hline & $(0.07)$ & $(0.07)$ & $(0.05)$ & $(0.04)$ & $(0.06)$ & $(0.05)$ \\
\hline Financial crisis & $0.83^{* * *}$ & $0.51 * * *$ & 0.26 & 0.08 & 0.08 & 0.01 \\
\hline & $(0.15)$ & $(0.14)$ & $(0.22)$ & $(0.11)$ & $(0.10)$ & $(0.10)$ \\
\hline Debt crisis & $0.81^{* * *}$ & $0.47^{* * * *}$ & 0.19 & 0.03 & 0.12 & 0.09 \\
\hline
\end{tabular}


Table 5 (continued)

\begin{tabular}{|c|c|c|c|c|c|c|}
\hline & $\mathrm{q} 0.01=\mathrm{q} 0.50$ & $\mathrm{q} 0.02=\mathrm{q} 0.50$ & $\mathrm{q} 0.05=\mathrm{q} 0.50$ & $\mathrm{q} 0.95=\mathrm{q} 0.50$ & $\mathrm{q} 0.98=\mathrm{q} 0.50$ & $\mathrm{q} 0.99=\mathrm{q} 0.50$ \\
\hline & $(0.15)$ & $(0.14)$ & $(0.14)$ & $(0.09)$ & $(0.11)$ & $(0.10)$ \\
\hline \multicolumn{7}{|l|}{ France-Hedged } \\
\hline \multirow[t]{2}{*}{ Not in crisis } & $0.48^{* * *}$ & $0.33^{*}$ & 0.14 & $0.19 * * *$ & $0.15^{*}$ & 0.03 \\
\hline & $(0.07)$ & $(0.17)$ & $(0.11)$ & $(0.05)$ & $(0.08)$ & $(0.12)$ \\
\hline \multirow[t]{2}{*}{ Financial crisis } & $-0.72^{* * *}$ & $-0.64 * *$ & -0.18 & 0.06 & 0.25 & $0.65^{* * *}$ \\
\hline & $(0.19)$ & $(0.26)$ & $(0.21)$ & $(0.10)$ & $(0.15)$ & $(0.22)$ \\
\hline \multirow[t]{2}{*}{ Debt crisis } & 0.10 & 0.15 & -0.12 & -0.15 & 0.02 & $-0.43^{* *}$ \\
\hline & $(0.10)$ & $(0.16)$ & $(0.16)$ & $(0.14)$ & $(0.22)$ & $(0.20)$ \\
\hline \multicolumn{7}{|c|}{ Germany - Hedged } \\
\hline \multirow[t]{2}{*}{ Not in crisis } & $0.34^{* * * *}$ & 0.21 & $0.13^{*}$ & $0.18^{* * *}$ & $0.24^{* * *}$ & $0.40^{* * *}$ \\
\hline & $(0.09)$ & $(0.19)$ & $(0.07)$ & $(0.05)$ & $(0.07)$ & $(0.10)$ \\
\hline \multirow[t]{2}{*}{ Financial crisis } & $-0.76^{* * *}$ & -0.49 & -0.23 & 0.10 & $0.22^{* *}$ & 0.09 \\
\hline & $(0.12)$ & $(0.31)$ & $(0.20)$ & $(0.09)$ & $(0.10)$ & $(0.11)$ \\
\hline \multirow[t]{2}{*}{ Debt crisis } & -0.10 & -0.13 & $-0.29 *$ & -0.08 & -0.30 & -0.27 \\
\hline & $(0.18)$ & $(0.22)$ & $(0.15)$ & $(0.11)$ & $(0.20)$ & $(0.26)$ \\
\hline \multicolumn{7}{|l|}{ Japan - Hedged } \\
\hline \multirow[t]{2}{*}{ Not in crisis } & $0.19^{* * *}$ & $0.14^{* * *}$ & $0.10^{* * * *}$ & -0.04 & -0.10 & -0.07 \\
\hline & $(0.05)$ & $(0.04)$ & $(0.03)$ & $(0.03)$ & $(0.06)$ & $(0.09)$ \\
\hline \multirow[t]{2}{*}{ Financial crisis } & 0.08 & -0.01 & -0.16 & $0.13^{* * *}$ & 0.17 & 0.08 \\
\hline & $(0.21)$ & $(0.62)$ & $(0.20)$ & $(0.05)$ & $(0.17)$ & $(0.109$ \\
\hline \multirow[t]{2}{*}{ Debt crisis } & 0.13 & 0.02 & -0.17 & $0.15^{* * * *}$ & $0.31^{* *}$ & $0.33^{* * *}$ \\
\hline & $(0.26)$ & $(0.63)$ & $(0.07)$ & $(0.05)$ & $(0.13)$ & $(0.11)$ \\
\hline \multicolumn{7}{|l|}{ UK - Hedged } \\
\hline \multirow[t]{2}{*}{ Not in crisis } & 0.00 & 0.13 & 0.01 & 0.00 & $0.18^{*}$ & 0.10 \\
\hline & $(0.19)$ & $(0.14)$ & $(0.07)$ & $(0.11)$ & $(0.11)$ & $(0.14)$ \\
\hline \multirow[t]{3}{*}{ Financial crisis } & $-0.27^{*}$ & $-0.34^{*}$ & 0.06 & $0.46^{* *}$ & 0.20 & 0.63 \\
\hline & $(0.15)$ & $(0.19)$ & $(0.18)$ & $(0.18)$ & $(0.14)$ & $(0.51)$ \\
\hline & & 0.19 & & & & \\
\hline \multirow[t]{2}{*}{ Debt crisis } & 0.43 & 0.12 & 0.14 & $0.39 * *$ & 0.08 & 0.36 \\
\hline & $(0.27)$ & $(0.57)$ & $(0.24)$ & $(0.17)$ & $(0.29)$ & $(0.36)$ \\
\hline
\end{tabular}

This table reports the test statistics for testing the equality of the slopes of the estimated coefficients. The results are displayed for six different cases of testing the equality between the following quantiles: (1) 0.01 and 0.50 quantiles; (2) 0.02 and 0.50 quantiles; (3) 0.05 and 0.50 quantiles; (4) 0.95 and 0.50 quantiles; (5) 0.98 and 0.50 quantiles; and (6) 0.99 and 0.50 quantiles. The tests are conducted for estimated coefficients $\beta, \gamma_{1}, \gamma_{2} ;$ denoted as Not in crisis, Financial crisis, and Debt crisis, respectively. Standard errors are given in parentheses. *, **, *** denote statistical significance at the $10 \%$, $5 \%$, and $1 \%$ levels, respectively.

et al., 2012; Piljak and Swinkels, 2017a; Chaieb et al., 2020). Reported results from a regional and country perspective of dependence analysis have important practical implications for diversification-seeking investors in international bond markets. Frontier bond markets have become attractive investment segment (making up a USD 100 billion market capitalization) and effective diversifier to global portfolios, as investors have directed attention towards frontier markets in a search for higher yields. ${ }^{15}$ However, despite attractive yields frontier bond markets represent higher risk level of sovereign default, as markets classified in frontier category have credit ratings of Ba1/BB + or lower by both Moody's and S\&P. ${ }^{16}$

Turning attention to the degree of dependence, the results show that the degree of dependence in normal times (average of $\beta$ coefficients across all quantiles) is around 0.51 for emerging markets USD-denominated return (aggregate index level). Furthermore, regional analysis confirms that the degree of dependence is very similar across regions, while on the country level the degree shows bigger variations and it ranges from 0.30 in Turkey to 0.83 in Brazil. ${ }^{17}$ In contrast to emerging markets, the degree of dependence for frontier markets bond return on the aggregate index level is very low and amounts to 0.09. This finding outlines that frontier bond markets have more considerable diversification potential than emerging bond markets and that international investors should not neglect them in asset-allocation decisions. Looking at the regional level, the results show that frontier markets in Latin America have

\footnotetext{
${ }^{15}$ See, for example, Bloomberg (20 December 2020): "Desperate need for yield pushes investors into frontier debt".https://www.bloomberg.com/ news/articles/2020-12-20/desperate-need-for-yield-pushes-investors-into-frontier-markets

${ }^{16}$ Certain markets (such as Egypt and Nigeria) included in NEXGEM Index have been labeled as "fallen angels" due to increased default risk and their earlier status of emerging market has been downgraded to frontier. Further example of a default risk is Côte d'Ivoire with an episode of bond default in 2011, after missing a $\$ 29$ million interest payment(https://www.imf.org/external/pubs/ft/fandd/2013/06/sy.htm). For more detailed discussion about frontier bond markets, see Piljak and Swinkels (2017a).

${ }^{17}$ See Appendix B (Table B.1) for degree of dependence for all emerging and frontier markets regions.
} 
Table 6

Test of asymmetry.

\begin{tabular}{|c|c|c|c|c|c|c|c|c|c|c|c|c|}
\hline & $\mathrm{q} 0.01=\mathrm{q} 0 . \varsigma$ & & $\mathrm{q} 0.02=\mathrm{q} 0.98$ & & $\mathrm{q} 0.03=\mathrm{q} 0.97$ & & $\mathrm{q} 0.04=\mathrm{q} 0.96$ & & $\mathrm{q} 0.05=\mathrm{q} 0.95$ & & $\mathrm{q} 0.10=\mathrm{q} 0.90$ & \\
\hline \multicolumn{13}{|c|}{ Panel A: Emerging markets } \\
\hline \multicolumn{13}{|c|}{ EMBI+ } \\
\hline No crisis & $0.54 * * *$ & $(0.20)$ & $0.33^{*}$ & $(0.17)$ & $0.36^{* *}$ & $(0.15)$ & $0.38 * *$ & $(0.15)$ & $0.28^{*}$ & $(0.16)$ & 0.18 & $(0.12)$ \\
\hline Financial & 0.15 & $(0.29)$ & $1.41^{* * *}$ & $(0.40)$ & $1.34^{* * *}$ & $(0.31)$ & $1.21^{* * *}$ & $(0.46)$ & 0.92 & $(0.60)$ & $0.83^{* *}$ & $(0.30)$ \\
\hline Debt & $-0.95 * *$ & $(0.39)$ & -0.33 & $(0.42)$ & -0.33 & $(0.40)$ & -0.38 & $(0.44)$ & 0.92 & $(0.60)$ & $-0.44 *$ & $(0.25)$ \\
\hline \multicolumn{13}{|c|}{ GBI EM Local Currency } \\
\hline No crisis & 0.12 & $(0.20)$ & 0.00 & $(0.17)$ & 0.14 & $(0.18)$ & 0.14 & $(0.15)$ & 0.15 & $(0.14)$ & 0.14 & $(0.11)$ \\
\hline Financial & -0.11 & $(0.39)$ & 0.04 & $(0.70)$ & -0.18 & $(0.72)$ & -0.15 & (1.13) & -0.18 & $(1.25)$ & -0.06 & $(0.38)$ \\
\hline Debt & $1.39^{* * *}$ & $(0.40)$ & $1.76^{* * * *}$ & $(0.34)$ & $1.14^{* *}$ & $(0.50)$ & 0.89 ** & $(0.44)$ & 0.72 & $(0.45)$ & 0.26 & $(0.34)$ \\
\hline \multicolumn{13}{|c|}{ GBI EM Local Currency - Hedged } \\
\hline No crisis & $0.32^{* *}$ & $(0.13)$ & 0.05 & $(0.09)$ & 0.04 & $(0.07)$ & 0.03 & $(0.06)$ & 0.02 & $(0.05)$ & 0.06 & $(0.04)$ \\
\hline Financial & 0.10 & $(0.17)$ & 0.07 & $(0.30)$ & 0.13 & $(0.15)$ & 0.14 & $(0.15)$ & 0.14 & $(0.16)$ & 0.14 & $(0.20)$ \\
\hline Debt & -0.05 & $(0.51)$ & -0.01 & $(0.45)$ & 0.12 & $(0.25)$ & 0.06 & $(0.17)$ & 0.04 & $(0.27)$ & -0.03 & $(0.17)$ \\
\hline \multicolumn{13}{|c|}{ Panel B: Frontier Markets } \\
\hline \multicolumn{13}{|c|}{ NEXGEM } \\
\hline No crisis & $0.60^{*}$ & $(0.31)$ & $0.35^{*}$ & $(0.20)$ & $0.42^{* *}$ & $(0.18)$ & $0.33^{* * *}$ & $(0.16)$ & 0.20 & $(0.16)$ & 0.05 & $(0.11)$ \\
\hline Financial & 0.18 & $(0.42)$ & 0.30 & $(0.42)$ & $0.90^{* *}$ & $(0.38)$ & $0.98^{* *}$ & $(0.37)$ & $0.69^{*}$ & $(0.37)$ & 0.32 & $(0.33)$ \\
\hline Debt & -1.72 & (9.56) & -1.09 & (7.41) & -1.22 & (8.03) & $-1.72^{* * *}$ & $(0.61)$ & -0.73 & (2.60) & -0.27 & $(0.39)$ \\
\hline \multicolumn{13}{|c|}{ Panel C: Developed Markets } \\
\hline \multicolumn{13}{|c|}{ France } \\
\hline No crisis & 0.17 & $(0.16)$ & 0.15 & $(0.15)$ & 0.23 & $(0.14)$ & 0.15 & $(0.13)$ & 0.08 & $(0.10)$ & 0.09 & $(0.09)$ \\
\hline Financial & 0.09 & $(0.32)$ & 0.21 & (3.49) & -0.08 & $(0.30)$ & -0.10 & $(0.29)$ & -0.05 & $(0.30)$ & -0.05 & $(0.27)$ \\
\hline Debt & $0.89^{* * *}$ & $(0.32)$ & 0.45 & $(0.36)$ & 0.19 & $(0.28)$ & 0.14 & $(0.24)$ & 0.07 & $(0.22)$ & 0.08 & $(0.19)$ \\
\hline \multicolumn{13}{|l|}{ Germany } \\
\hline No crisis & 0.21 & $(0.13)$ & 0.03 & $(0.11)$ & 0.00 & $(0.11)$ & -0.04 & $(0.09)$ & -0.02 & $(0.11)$ & 0.09 & $(0.07)$ \\
\hline Financial & -0.08 & $(0.30)$ & 0.42 & $(0.28)$ & 0.02 & $(0.28)$ & 0.02 & $(0.28)$ & -0.03 & $(0.29)$ & -0.04 & $(0.24)$ \\
\hline Debt & $0.54 *$ & $(0.28)$ & 0.39 & $(0.30)$ & 0.17 & $(0.30)$ & 0.07 & $(0.31)$ & 0.08 & $(0.27)$ & -0.15 & $(0.19)$ \\
\hline \multicolumn{13}{|l|}{ Japan } \\
\hline No crisis & $0.65^{* * *}$ & $(0.17)$ & 0.52 & $(0.19)$ & $0.42^{* *}$ & $(0.16)$ & $0.47^{* * *}$ & $(0.16)$ & $0.53^{* * *}$ & $(0.15)$ & $0.39^{* * *}$ & (0.13) \\
\hline Financial & -0.09 & (1.00) & 0.08 & (1.15) & -0.22 & $(0.48)$ & -0.17 & $(0.46)$ & -0.19 & $(0.46)$ & -0.05 & (0.50) \\
\hline Debt & -0.83 & $(0.52)$ & -0.32 & $(0.61)$ & -0.68 & $(0.46)$ & -0.53 & $(0.48)$ & -0.52 & $(0.48)$ & -0.29 & $(0.37)$ \\
\hline \multicolumn{13}{|l|}{ UK } \\
\hline No crisis & 0.19 & $(0.34)$ & 0.11 & $(0.20)$ & 0.16 & $(0.16)$ & 0.12 & $(0.14)$ & 0.15 & $(0.14)$ & 0.07 & $(0.14)$ \\
\hline \multirow[t]{2}{*}{ Financial } & -0.42 & (0.99) & -0.41 & $(0.47)$ & -0.32 & $(0.39)$ & -0.28 & $(0.45)$ & -0.39 & $(0.48)$ & -0.17 & $(0.38)$ \\
\hline & & & & & & & & & & & (continued & xt page) \\
\hline
\end{tabular}


Table 6 (continued)

\begin{tabular}{|c|c|c|c|c|c|c|c|c|c|c|c|c|}
\hline & \multicolumn{2}{|c|}{$\mathrm{q} 0.01=\mathrm{q} 0.99$} & \multicolumn{2}{|c|}{$\mathrm{q} 0.02=\mathrm{q} 0.98$} & \multicolumn{2}{|c|}{$\mathrm{q} 0.03=\mathrm{q} 0.97$} & \multicolumn{2}{|c|}{$\mathrm{q} 0.04=\mathrm{q} 0.96$} & \multicolumn{2}{|c|}{$\mathrm{q} 0.05=\mathrm{q} 0.95$} & \multicolumn{2}{|c|}{$\mathrm{q} 0.10=\mathrm{q} 0.90$} \\
\hline Debt & 1.15 & $(0.92)$ & 0.20 & $(0.88)$ & -0.21 & $(0.34)$ & -0.08 & $(0.36)$ & -0.19 & $(0.38)$ & 0.33 & $(0.37)$ \\
\hline \multicolumn{13}{|l|}{ USA } \\
\hline No crisis & $-0.45^{* * *}$ & $(0.09)$ & $-0.29 * * *$ & $(0.10)$ & $-0.23^{* *}$ & $(0.08)$ & $-0.16^{* *}$ & $(0.08)$ & $-0.18^{* *}$ & $(0.07)$ & $-0.13^{*}$ & $(0.06)$ \\
\hline Financial & $0.83^{* * * *}$ & $(0.19)$ & $0.43^{* *}$ & $(0.18)$ & 0.27 & $(0.23)$ & 0.16 & $(0.24)$ & 0.18 & $(0.25)$ & -0.08 & $(0.14)$ \\
\hline Debt & $0.73^{* * * *}$ & $(0.20)$ & $0.35^{*}$ & $(0.19)$ & 0.30 & $(0.22)$ & 0.17 & $(0.19)$ & 0.16 & $(0.18)$ & 0.18 & $(0.13)$ \\
\hline \multicolumn{13}{|c|}{ France-Hedged } \\
\hline No crisis & $0.45^{* * *}$ & $(0.14)$ & 0.18 & $(0.19)$ & -0.03 & $(0.28)$ & -0.10 & $(0.27)$ & -0.05 & $(0.12)$ & -0.03 & $(0.07)$ \\
\hline Financial & $-1.37^{* * * *}$ & $(0.31)$ & $-0.89^{* * *}$ & $(0.31)$ & -0.49 & $(0.39)$ & -0.29 & $(0.37)$ & -0.25 & $(0.24)$ & -0.07 & $(0.15)$ \\
\hline Debt & $0.54^{* *}$ & $(0.25)$ & 0.13 & $(0.28)$ & 0.26 & $(0.43)$ & 0.24 & $(0.35)$ & 0.03 & $(0.23)$ & -0.03 & $(0.17)$ \\
\hline \multicolumn{13}{|c|}{ Germany - Hedged } \\
\hline No crisis & -0.06 & $(0.13)$ & -0.03 & $(0.21)$ & -0.16 & $(0.19)$ & -0.05 & $(0.09)$ & -0.05 & $(0.09)$ & -0.09 & $(0.07)$ \\
\hline Financial & $-0.85^{* * *}$ & $(0.18)$ & $-0.71^{* *}$ & (0.33) & -0.44 & $(0.27)$ & -0.40 & $(0.22)$ & -0.33 & $(0.23)$ & -0.05 & $(0.14)$ \\
\hline Debt & 0.17 & $(0.33)$ & 0.17 & $(0.31)$ & -0.03 & $(0.29)$ & -0.29 & $(0.21)$ & -0.21 & $(0.21)$ & 0.04 & $(0.24)$ \\
\hline \multicolumn{13}{|c|}{ Japan - Hedged } \\
\hline No crisis & $0.26^{* *}$ & $(0.10)$ & 0.15 & $(0.15)$ & $0.15^{* *}$ & $(0.05)$ & $0.16^{* * * *}$ & $(0.05)$ & 0.08 & $(0.10)$ & $0.07^{* *}$ & $(0.04)$ \\
\hline Financial & 0.00 & $(0.23)$ & 0.21 & (3.49) & -0.33 & $(0.22)$ & $-0.39 * * *$ & $(0.12)$ & -0.05 & $(0.30)$ & $-0.23^{*}$ & $(0.07)$ \\
\hline Debt & -0.20 & $(0.28)$ & 0.45 & $(0.36)$ & -0.30 & $(0.27)$ & $-0.36^{* * *}$ & $(0.10)$ & 0.07 & $(0.22)$ & $-0.08^{*}$ & $(0.07)$ \\
\hline \multicolumn{13}{|c|}{ UK - Hedged } \\
\hline No crisis & -0.10 & $(0.24)$ & -0.05 & $(0.18)$ & -0.05 & $(0.17)$ & -0.09 & $(0.14)$ & 0.01 & (0.13) & 0.08 & $(0.10)$ \\
\hline Financial & $-0.91^{*}$ & (0.54) & $-0.54 * *$ & $(0.26)$ & -0.35 & $(0.30)$ & -0.48 & $(0.40)$ & -0.39 & $(0.26)$ & -0.37 & $(0.48)$ \\
\hline Debt & 0.06 & $(0.46)$ & 0.04 & (0.64) & 0.08 & $(0.36)$ & -0.07 & $(0.30)$ & -0.25 & $(0.30)$ & -0.39 & $(0.21)$ \\
\hline
\end{tabular}

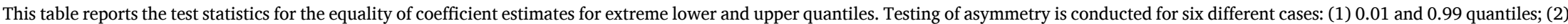

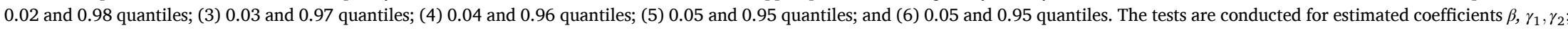
denoted as No crisis, Financial (crisis), and Debt (crisis), respectively. Standard errors are given in parentheses. *.**.*** denote statistical significance at the $10 \% .5 \%$. and $1 \%$ levels, respectively. 
Table 7

Degree of dependence for different bond maturities (short, medium, and long).

\begin{tabular}{|c|c|c|c|}
\hline & $\begin{array}{l}\beta \\
\text { Not in crisis }\end{array}$ & $\gamma_{1}$ Financial crisis & $\gamma_{2}$ Debt crisis \\
\hline \multicolumn{4}{|c|}{ Panel A: Emerging Markets (local currency) } \\
\hline \multicolumn{4}{|c|}{ GBI EM - No Hedge } \\
\hline Short & 0.95 & -0.10 & -0.04 \\
\hline Medium & 1.07 & -0.15 & -0.04 \\
\hline Long & 1.29 & -0.40 & -0.25 \\
\hline \multicolumn{4}{|c|}{ GBI EM - Hedged } \\
\hline Short & 0.08 & 0.03 & -0.01 \\
\hline Medium & 0.25 & -0.04 & -0.02 \\
\hline Long & 0.46 & -0.20 & -0.12 \\
\hline \multicolumn{4}{|c|}{ Panel B: Developed Markets } \\
\hline \multicolumn{4}{|c|}{ Not Hedged } \\
\hline \multicolumn{4}{|c|}{ France } \\
\hline Short & 1.39 & -0.01 & 0.24 \\
\hline Medium & 1.61 & 0.00 & 0.22 \\
\hline Long & 2.07 & -0.15 & 0.16 \\
\hline \multicolumn{4}{|l|}{ Germany } \\
\hline Short & 1.38 & -0.02 & 0.19 \\
\hline Medium & 1.62 & -0.02 & 0.15 \\
\hline Long & 2.08 & -0.27 & -0.09 \\
\hline \multicolumn{4}{|l|}{ Japan } \\
\hline Short & 1.26 & -0.06 & 0.06 \\
\hline Medium & 1.37 & -0.03 & 0.08 \\
\hline Long & 1.59 & -0.15 & 0.01 \\
\hline \multicolumn{4}{|l|}{ UK } \\
\hline Short & 1.06 & -0.41 & -0.13 \\
\hline Medium & 1.32 & -0.44 & -0.07 \\
\hline Long & 1.85 & -0.73 & -0.37 \\
\hline \multicolumn{4}{|l|}{ US } \\
\hline Short & 0.22 & 0.07 & -0.05 \\
\hline Medium & 0.66 & 0.03 & -0.10 \\
\hline Long & 1.24 & -0.07 & -0.31 \\
\hline \multicolumn{4}{|l|}{ Hedged } \\
\hline \multicolumn{4}{|l|}{ France } \\
\hline Short & 0.12 & 0.06 & 0.00 \\
\hline Medium & 0.37 & 0.08 & -0.01 \\
\hline Long & 0.81 & -0.05 & -0.12 \\
\hline \multicolumn{4}{|l|}{ Germany } \\
\hline Short & 0.12 & 0.05 & -0.05 \\
\hline Medium & 0.37 & 0.06 & -0.09 \\
\hline Long & 0.84 & -0.17 & -0.31 \\
\hline \multicolumn{4}{|l|}{ Japan } \\
\hline Short & 0.03 & 0.05 & -0.01 \\
\hline Medium & 0.14 & 0.09 & 0.04 \\
\hline Long & 0.32 & 0.00 & 0.00 \\
\hline \multicolumn{4}{|l|}{ UK } \\
\hline Short & 0.15 & 0.09 & 0.00 \\
\hline Medium & 0.41 & 0.12 & 0.02 \\
\hline Long & 0.94 & -0.21 & -0.30 \\
\hline
\end{tabular}

This table reports the average degree of dependence for different bond maturities: short (1-5 years), medium (5-10 years), and long (15 + years) for emerging (Panel A) and developed markets (Panel B). The degree of dependence is given by the average coefficient estimates across all quantiles according to the model defined by Equation (1). The table shows the average degree of dependence in the non-crisis period ( $\beta$ ), change in the degree of dependence due to the global financial crisis $\left(\gamma_{1}\right)$ and change in the degree of dependence due to the Eurozone debt crisis $\left(\gamma_{2}\right)$.

the lowest degree of dependence in the period before the crisis and hence the highest diversification potential. However, the degree of dependence is increasing during both crises events, consistent with the contagion and observed patterns in the structure with a large magnitude of dependence in both left and right tail during crises. However, looking deeper at the country level shows that there are differences across countries in the sign of change (e.g. positive for Dominican Republic and Pakistan, but negative for Ecuador and Egypt).

For emerging bond markets on the aggregate level there is a negative change during both the global financial crisis and the Eurozone debt crisis. This pattern is consistent with decoupling and there are two intuitive explanations for this finding. First, emerging markets bonds are viewed as "equity-like assets". Second, their correlation with the global bond market portfolio (mainly consisting of developed markets bonds) is decreasing because developed markets bonds are safe assets (Piljak, 2013). The same pattern of negative change is observed across regions as well, with the European region having the smallest $(-0.009)$ and Asian region the highest negative change $(-0.256)$ during the global financial crisis. The observed regional difference indicates that emerging market 
Panel A: Local currency emerging bond markets: short, medium, and long maturities
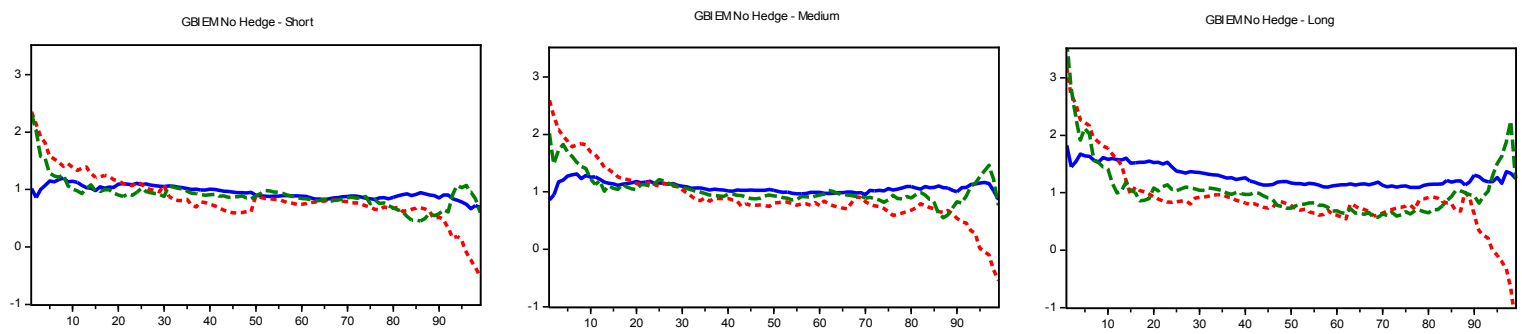

GBIEMHedged-Short
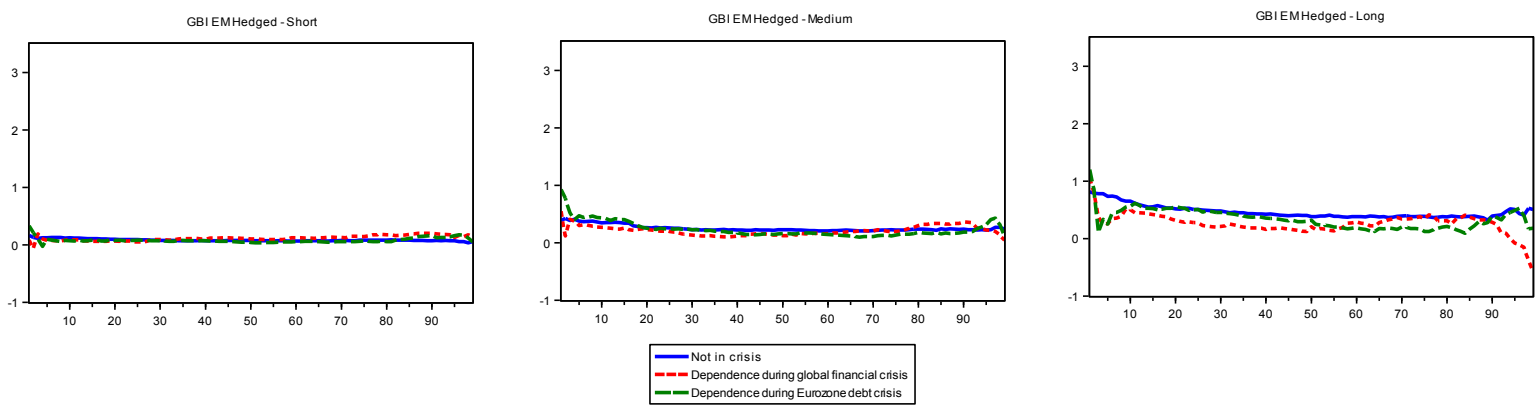

Panel B: Developed markets (represented by the UK): short, medium and long maturities
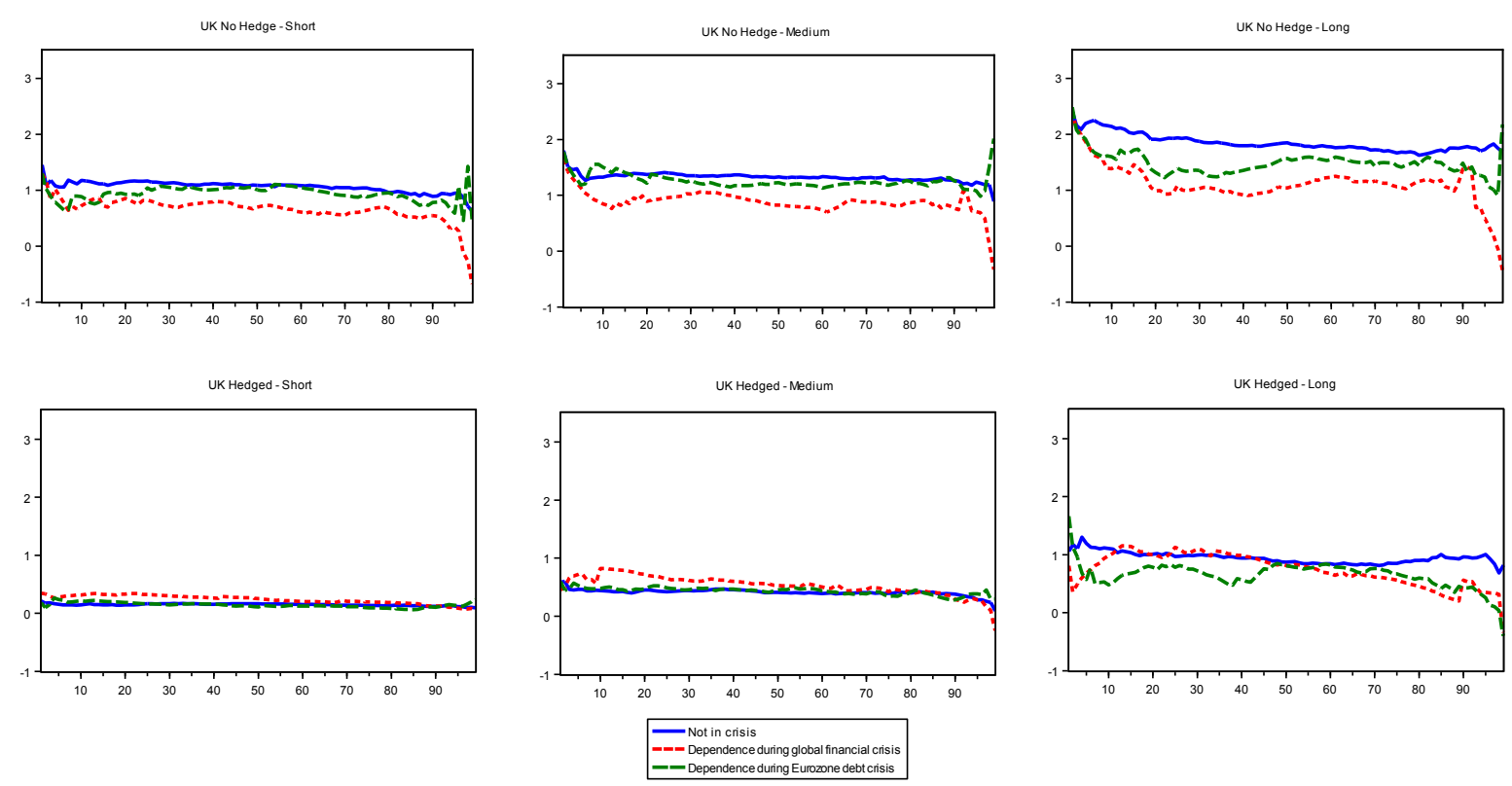

Fig. 4. Structure of dependence for different bond maturities. This figure shows dependence structure of emerging and developed markets bond returns on the returns of global bond market portfolio in case of three different bond maturities: short (1-5 years), medium (5-10 years), and long (15 + years). Panel A refers to dependence in emerging markets local-currency bond returns (graphs on upper level show the case of unhedged returns and graph on lower level shows returns hedged to US dollar using currency derivatives). Panel B refers to dependence in the UK as a representative of developed markets (graphs on upper level show the case of unhedged returns and graph on lower level shows returns hedged to US dollar using currency derivatives). Description of lines and axes is the same as in Fig. 2.

bonds from European countries are perceived as safer than those of Asian countries. However, the magnitude of change in dependence due to the Eurozone debt crisis is very similar in all regions.

Focusing to the frontier markets results, we show that despite a noticeable change in the degree of dependence in both crises events, the overall level of dependence is still much lower than for emerging markets. This finding provides evidence that frontier bond markets might yet have important diversification potential even during crisis events. This is also in line with literature pointing out that frontier bond markets have lower integration level with global markets (Piljak and Swinkels, 2017a). The regional analysis further 
Table 8

Contagion and flight-to-quality (global stock and bond markets).

\begin{tabular}{|c|c|c|c|c|c|}
\hline & Coefficient & S.E. & z-Statistics & p-Value & \\
\hline \multicolumn{6}{|c|}{ Panel A: Emerging Markets } \\
\hline \multicolumn{6}{|c|}{ EMBI +} \\
\hline Rs & 0.227 & 0.014 & 15.370 & 0.00 & \\
\hline Rs $D_{\text {fincrisis }}$ & 0.147 & 0.017 & 8.288 & 0.00 & Contagion \\
\hline Rs D $D_{\text {debtcrisis }}$ & -0.059 & 0.025 & -2.303 & 0.02 & Decoupling \\
\hline Constant & 0.186 & 0.027 & 6.757 & 0.00 & \\
\hline \multicolumn{6}{|c|}{ GBI EM Local Currency } \\
\hline Rs & 0.253 & 0.017 & 14.818 & 0.00 & \\
\hline Rs $D_{\text {fincrisis }}$ & 0.082 & 0.019 & 4.211 & 0.00 & Contagion \\
\hline Rs $D_{\text {debtcrisis }}$ & 0.068 & 0.024 & 2.804 & 0.00 & Contagion \\
\hline Constant & 0.109 & 0.029 & 3.762 & 0.00 & \\
\hline \multicolumn{6}{|c|}{ GBI EM Local Currency - Hedged } \\
\hline Rs & 0.037 & 0.005 & 6.497 & 0.00 & \\
\hline Rs $D_{\text {fincrisis }}$ & 0.019 & 0.007 & 2.628 & 0.00 & Contagion \\
\hline Rs $D_{\text {debtcrisis }}$ & -0.021 & 0.008 & -2.445 & 0.01 & Decoupling \\
\hline Constant & 0.081 & 0.010 & 7.988 & 0.00 & \\
\hline \multicolumn{6}{|c|}{ Panel B: Frontier Markets } \\
\hline \multicolumn{6}{|c|}{ NEXGEM } \\
\hline Rs & 0.077 & 0.017 & 4.388 & 0.00 & \\
\hline Rs $D_{\text {fincrisis }}$ & -0.028 & 0.029 & -0.970 & 0.33 & - \\
\hline Rs $D_{\text {debtcrisis }}$ & 0.009 & 0.025 & 0.371 & 0.71 & _- \\
\hline Constant & 0.239 & 0.033 & 7.171 & 0.00 & \\
\hline \multicolumn{6}{|c|}{ Panel C: Developed Markets } \\
\hline \multicolumn{6}{|c|}{ GBI - Non Hedged } \\
\hline Rs & -0.011 & 0.018 & -0.608 & 0.54 & \\
\hline Rs $D_{\text {fincrisis }}$ & -0.016 & 0.029 & -0.564 & 0.57 & - \\
\hline Rs $D_{\text {debtcrisis }}$ & 0.046 & 0.035 & 1.295 & 0.19 & - \\
\hline Constant & 0.080 & 0.032 & 2.482 & 0.01 & \\
\hline \multicolumn{6}{|l|}{ GBI - Hedged } \\
\hline Rs & -0.062 & 0.008 & -7.574 & 0.00 & \\
\hline Rs $D_{\text {fincrisis }}$ & 0.024 & 0.009 & 2.493 & 0.01 & Decoupling \\
\hline Rs $D_{\text {debtcrisis }}$ & -0.005 & 0.012 & -0.427 & 0.66 & - \\
\hline Constant & 0.099 & 0.013 & 7.223 & 0.00 & \\
\hline
\end{tabular}

This table reports the estimation results from the regression model in Eq.2 for emerging (Panel A), frontier (Panel B), and developed markets (Panel C). $R_{s}$ denotes the returns of the global stock market index, $R_{s} D_{\text {fincrisis }}$ is the interaction term of the global stock market return and the global financial crisis period, while $R_{s} D_{\text {debtcrisis }}$ is the interaction term of the global stock market return and the Eurozone debt crisis period. Definitions of contagion and decoupling are given in Section 5.6.

shows that there are differences in the magnitude of crisis effects (for instance, African region appeared to be less vulnerable to crises in comparison to Latin American region), which confirms previous finding that frontier markets should not be perceived by investors as homogenous groups of markets.

\subsection{Dependence in developed bond markets}

The developed markets return dependence is rather flat over quantiles in the non-crisis period, no matter whether hedged or unhedged returns are considered (as shown in Fig. 3). France and Germany (unhedged returns) have a slight U-shape pattern for the Eurozone debt crisis. Japan is less affected by the Eurozone debt crisis. The dependence pattern for the US returns is rather flat in noncrisis, but during both crises periods, there is a left tail dependence. The degree of dependence in normal times (average of $\beta$ coefficients across all quantiles) is in the range from 1.37 (Japan) to 1.61 (France) for unhedged returns, while the corresponding range for hedged returns is from 0.13 (Japan) to 0.56 (the UK). This result shows that the degree of dependence is much higher for unhedged than for hedged returns, which is a similar finding as in the local currency emerging bond markets. Hedged and unhedged returns also show different patterns with regard to changes in the degree of dependence during crises episodes. In particular, during the Eurozone debt crisis the degree is decreasing for hedged returns, which is consistent with decoupling, while at the same time the degree is increasing for unhedged returns (except UK and US), consistent with contagion. This finding illustrates that unhedged bond returns were more vulnerable to the Eurozone debt crisis than hedged bond returns.

\subsection{Tests for inter-quantile differences and asymmetric dependence}

Table 5 displays the results of the significance test of inter-quantile differences. In particular, we test whether the slopes of the 
Table 9

Portfolio analysis.

\begin{tabular}{|c|c|c|c|c|c|c|}
\hline & GABI & GBI-EM & EMBI+ & NEXGEM & GBI & DIV \\
\hline Average & 5.21 & 7.79 & 9.05 & 10.79 & 5.24 & 8.21 \\
\hline \multirow[t]{3}{*}{ Volatility } & 5.74 & 11.87 & 9.58 & 9.66 & 6.97 & 7.42 \\
\hline & \multicolumn{6}{|c|}{ Worst 10 weekly returns of GABI } \\
\hline & GABI & GBI-EM & EMBI+ & NEXGEM & GBI & DIV \\
\hline 21-Jun-13 & -2.69 & -6.74 & -5.90 & -2.87 & -2.85 & -4.59 \\
\hline 13-Jun-08 & -2.68 & -2.10 & -1.22 & -0.79 & -3.06 & -1.79 \\
\hline 10-Nov-16 & -2.33 & -5.26 & -2.44 & -1.97 & -2.81 & -3.12 \\
\hline 1-Aug-03 & -2.29 & -1.63 & -3.84 & -3.37 & -2.32 & -2.79 \\
\hline 18-Nov-16 & -2.23 & -2.28 & -1.97 & -1.36 & -2.68 & -2.07 \\
\hline 23-Jan-09 & -2.10 & -3.04 & -1.01 & 0.50 & -2.47 & -1.50 \\
\hline 21-Mar-03 & -2.07 & -0.73 & -0.74 & -0.03 & -2.63 & -1.03 \\
\hline 7-Jan-05 & -1.93 & -4.19 & -1.01 & -0.40 & -2.40 & -2.00 \\
\hline 6-Mar-15 & -1.91 & -4.69 & -0.91 & 0.12 & -2.16 & -1.91 \\
\hline 6-Nov-15 & -1.85 & -1.59 & -0.43 & 0.05 & -2.33 & -1.07 \\
\hline \multirow[t]{3}{*}{ Average } & -2.21 & -3.22 & -1.95 & -1.01 & -2.57 & -2.19 \\
\hline & \multicolumn{6}{|c|}{ Worst 10 weekly returns of GABI during $G F C$} \\
\hline & GABI & GBI-EM & EMBI+ & NEXGEM & GBI & DIV \\
\hline 13-Jun-08 & -2.68 & -2.10 & -1.22 & -0.79 & -3.06 & -1.79 \\
\hline 18-Apr-08 & -1.76 & -0.18 & -0.29 & 0.29 & -2.19 & -0.59 \\
\hline 3-Oct-08 & -1.53 & -4.22 & -2.86 & -6.12 & -1.47 & -3.67 \\
\hline 30-May-08 & -1.48 & -0.49 & -0.16 & -0.66 & -1.69 & -0.75 \\
\hline 8-Aug-08 & -1.47 & -3.10 & -0.28 & -0.16 & -1.89 & -1.36 \\
\hline 31-Oct-08 & -1.44 & 9.28 & 13.65 & -1.48 & -2.04 & 4.85 \\
\hline $10-$ Oct-08 & -1.19 & -9.34 & -13.82 & -11.59 & -0.16 & -8.73 \\
\hline 14-Dec-07 & -1.14 & -1.55 & -0.23 & 0.53 & -1.40 & -0.66 \\
\hline 8-Feb-08 & -0.91 & -2.11 & -1.27 & 0.26 & -1.11 & -1.06 \\
\hline 18-Jul-08 & -0.89 & 2.18 & 0.41 & -0.60 & -0.66 & 0.33 \\
\hline \multirow[t]{2}{*}{ Average } & -1.45 & -1.17 & -0.61 & -2.03 & -1.57 & -1.34 \\
\hline & \multicolumn{6}{|c|}{ Worst 10 weekly returns of GABI during $E D C$} \\
\hline Date & GABI & GBI-EM & EMBI+ & NEXGEM & GBI & DIV \\
\hline 12-Nov-10 & -1.72 & -1.98 & -2.08 & -1.72 & -1.93 & -1.93 \\
\hline 7-Jan-11 & -1.52 & -0.70 & 0.60 & 0.51 & -1.94 & -0.38 \\
\hline 25-Nov-11 & -1.45 & -4.13 & -1.17 & -1.11 & -1.68 & -2.02 \\
\hline 26-Nov-10 & -1.39 & -3.27 & -0.16 & -0.23 & -1.62 & -1.32 \\
\hline 10-Dec-10 & -1.26 & -1.06 & -0.86 & -0.39 & -1.47 & -0.94 \\
\hline 7-May-10 & -1.26 & -6.69 & -2.72 & -4.05 & -1.12 & -3.64 \\
\hline 13-Aug-10 & -1.23 & -1.09 & 0.70 & 0.77 & -1.51 & -0.28 \\
\hline 4-Dec-09 & -1.15 & 1.32 & -0.17 & -0.64 & -1.52 & -0.25 \\
\hline 9-Sep-11 & -0.98 & -2.90 & -0.23 & 0.10 & -1.11 & -1.03 \\
\hline 28-May-10 & -0.98 & 1.47 & 0.88 & 0.99 & -1.10 & 0.56 \\
\hline Average & -1.29 & -1.90 & -0.52 & -0.58 & -1.50 & -1.13 \\
\hline
\end{tabular}

This table reports the 10 worst weekly returns in the global aggregate bond market (GABI) over the entire sample period, during the global financial crisis, and during the Eurozone debt crisis, as well as the returns of other asset classes and of a naively diversified portfolio (DIV) of unhedged government bonds.

estimated coefficients change across quantiles. The same methodological framework is utilized in Pires et al. (2015) and Boubaker et al. (2019). We focus on the extreme quantiles on the left (1st, 2nd, and 5th quantile) and right (95th, 98th, and 99th quantile) side of the distribution and test their equality to the median quantile. For USD-denominated emerging markets, the estimated slopes are significantly different from each other in the case where extreme quantile on the left is compared to the median quantile (1st and 50 th quantile) in all time periods (non-crisis, global financial crisis, debt crisis). It is interesting to note that during the Eurozone debt crisis, each quantile is statistically significantly different from the median, except the 5 th quantile.

Regarding local currency emerging bond markets, the test shows different patterns between hedged and unhedged subsamples. In the hedged subsample, the only statistically significant inter-quantile difference at $5 \%$ level is noticed in the no-crisis period. In contrast, in the non-hedged subsample, the most significant difference is shown during the debt crisis. On the other hand, frontier markets exhibit a highly statistically significant (at 1\% level) inter-quantile difference in all six tested cases during the financial crisis and in all right-hand side extreme test cases during the debt crisis. For the developed markets, the main overall pattern is that hedged returns exhibit more statistically significant inter-quantile differences comparing to non-hedged returns, primarily in the non-crisis period. During the global financial and debt crises, there are almost no significant inter-quantile differences, with the notable exception of the USA for the test concerning extreme left-hand side and median inter-quantile difference cases.

The next question is whether there is an asymmetric impact in negative and positive bond market conditions. ${ }^{18}$ In this respect, we

\footnotetext{
${ }^{18}$ For further information on the importance of asymmetric dependence in financial markets please see Ang and Chen (2002), Yuan (2005), and Baur and Schulze (2009) among others.
} 
perform tests over a range of quantiles (quantile 1-10 versus 90-99) to test whether the impact is equal in extreme lower and higher quantiles. Table 6 presents the results of the test for asymmetry in six different cases (1st and 99th, 2nd and 98th, 3rd and 97th, 4th and 96th, 5th and 95th' and 10th and 90th). ${ }^{19}$ Following Baur and Schulze (2009), we focus on the interpretation of asymmetry results in the case of the most extreme quantiles (1st and 99th; and 2nd and 98th). The null hypothesis of "no asymmetry" can be rejected in the case of USD-denominated emerging markets in all periods (non-crisis, global financial crisis, debt crisis). For the local currency emerging bond markets, the asymmetry is present for non-hedged returns only during the Eurozone debt crisis and for hedged returns only in the non-crisis period. Frontier markets show little statistical significance (at 10\%) only in the non-crisis period. Among developed markets, the only notable asymmetry in all periods (non-crisis, global financial crisis, debt crisis) is present in the US and France (hedged returns). The asymmetry tests in other than two of the most extreme quantiles do not show statistical significance in the majority of the markets, with certain exceptions such as Japan (hedged and unhedged returns) and USD-denominated (emerging and frontier) markets in some cases.

\subsection{The role of bond maturity}

In this section we extend the analysis by examining the role of bond maturities. In particular, we investigate differences in degree and structure of dependence between short-, medium-, and long-term bond maturities. Furthermore, we analyze whether changes in degree or structure of dependence during the global financial crisis and the Eurozone debt crisis are more prevalent in long-term bonds than in short-term bonds. For this purpose, we use short-term (1-5 years), medium-term (5-10 years), and long-term (15+ years) bond indices of local currency-denominated emerging and developed markets. ${ }^{20}$

The results for degree of dependence are reported in Table 7. All analyzed markets exhibit the same pattern during non-crisis period that degree of dependence is increasing as maturity is increasing. Regarding the change in the degree of dependence during the global financial crisis and the Eurozone debt crisis, there is a clear pattern for emerging markets which shows that the change is larger for longer maturities. A similar pattern is observed for most of developed markets during the global financial crisis, but during the Eurozone debt crisis there is a deviation from this pattern, especially for unhedged returns. With respect to hedging perspective, the results show that the degree of dependence is higher for unhedged returns than for hedged across emerging and developed markets.

Fig. 4 shows the structure of dependence for different bond maturities in emerging (Panel A) and developed markets (Panel B). ${ }^{21}$ The results in both panels confirm the previous finding that dependence and crises-induced changes of dependence are higher for unhedged returns than for hedged. Furthermore, the changes in the structure of dependence are more prevalent in long-term bonds than in short- and medium-term bonds for both emerging and developed markets.

\subsection{Contagion and flight-to-quality}

This section examines contagion and flight-to-quality from global stock market to bond markets. We follow the econometric framework proposed by Baur and Lucey (2009). The first part of the framework consists of estimation of the time-varying stock-bond correlations using the dynamic conditional correlation (DCC) model proposed by Engle (2002), while the second part consists of testing for flights-to-quality and contagion. Baur and Lucey (2009) define contagion between stocks and bonds as a significant increase of the correlation coefficient during crisis period (compared to a benchmark period) that results in a positive correlation level; while flight-toquality from stocks to bonds is defined as a significant decrease of the correlation coefficient during crisis period compared to a benchmark period that results in a negative correlation level. The basic model to test for changes in the level of stock-bond correlation is specified as follows:

$$
R_{b, t}=\alpha+\beta R_{s, t}+\gamma_{1} R_{s, t} D_{\text {fincrisis }, t}+\gamma_{2} R_{s, t} D_{\text {debtcrisis }, t}+e_{t}
$$

where $R_{b, t}$ denotes the returns of government bond market (emerging, frontier, developed) at time $t$, and $R_{s, t}$ denotes the returns of the global stock market index at time $t$. $D_{\text {fincrisis }}$ is a dummy variable capturing the global financial crisis period; $D_{\text {debtcrisis }}$ is a dummy variable capturing the Eurozone debt crisis period, $R_{s} D_{\text {fincrisis }}$ is the interaction term of the global stock market return and the global financial crisis period, while $R_{s} D_{\text {debtcrisis }}$ is the interaction term of the global stock market return and the Eurozone debt crisis period. Similarly to Baur and Lucey (2009), the model is estimated with a GARCH $(1,1)$ model specification to take into account autocorrelation and heteroscedasticity.

The sum of the parameters $\beta$ and $\gamma_{1}$ determines the level of stock-bond correlation in the global financial crisis period, while the sum of the parameters $\beta$ and $\gamma_{2}$ determines the level of stock-bond correlation in the Eurozone debt crisis period. The $\gamma_{1}$ and $\gamma_{2}$ represent change of stock-bond correlation compared to the benchmark correlation given by $\beta$. If $\gamma_{1}$ is significantly positive and also the sum of $\beta$ and $\gamma_{1}$ is significantly positive, there is a contagion between stocks and bonds during the global financial crisis (the same

\footnotetext{
19 The results for remaining four cases (6th and 94th, 7th and 93th, 8th and 92th, and 9th and 91th) are not shown in the Table 6 in order to conserve space.

${ }^{20}$ For the frontier markets (USD- denominated bonds) there are no available data for different maturities. The inclusion criteria for bonds in NEXGEM Index require that bonds have remaining maturity longer than 2.5 years.

21 In order to conserve the space, in panel B of the Fig. 4 we show results only for the UK as a representative of the developed markets. The results for other developed countries are available in an Online Appendix.
} 
applies to case of $\gamma_{2}$ and the Eurozone debt crisis). On contrary, if $\gamma_{1}$ is significantly negative and also the sum of $\beta$ and $\gamma_{1}$ is significantly negative, there is flight-to-quality from stocks to bonds. However, if there is a significant negative change in the correlation $\left(\gamma_{1}\right)$ but the level of correlation (sum of $\beta$ and $\gamma_{1}$ ) is positive, there is decoupling between stocks and bonds, but there is no flight-to-quality.

The estimated stock-bond correlations are shown in Appendix D. For the local-currency emerging bond markets (Fig. D.1), the graph shows that correlation with global stock market is mainly positive throughout the sample time, which is consistent with "equitylike" properties of EM local bonds (bond returns are moving in the same direction as global stock market returns). The exception from the main pattern is a short period of negative correlations in 2002 and 2011 for hedged returns. The episode of negative correlations in 2011 corresponds with Eurozone debt crisis period. The correlations of non-hedged returns are higher than for hedged returns. For the USD-denominated emerging bond markets (EMBI+), shown in Fig. D.3, the correlations are also positive throughout the sample time, confirming "equity-like" properties observed in local-currency EM bonds. The pattern of positive correlation observed on aggregate EMBI+ level is very similar for regional level (the only exceptions with very short period of negative correlations are African and Asian region). Frontier markets (Fig. D.4) exhibit the pattern of varying correlations with longer periods of positive correlation that is followed with shorter periods of negative correlations. Periods of negative correlations indicate decoupling from the global stock market returns.

The results for developed markets (Fig. D.2) indicate that correlations of non-hedged returns are higher than for hedged returns (same pattern as for EM local bonds). The correlations of hedged returns are negative throughout the sample time (this is consistent with decoupling where bonds of developed markets are seen as safe assets and they move in opposite direction from global stock market returns). The correlations of non-hedged returns are varying with longer periods of positive correlation that is followed with shorter periods of negative correlations. Negative correlations are observed especially during the global financial crisis.

The estimation results from Eq. (2) are reported in Table 8. The results show that during the global financial crisis there has been contagion from the global stock market to all segments of emerging bond markets (USD-denominated, local currency hedged and nonhedged). Since the global financial crisis originated from stock markets, the finding of contagion confirms "equity-like" properties of emerging markets bonds, which is consistent with Panchenko and Wu (2009) and Dimic et al. (2016). On the contrary, during the Eurozone debt crisis (originated from bond markets) there has been decoupling from global stock markets in case of USD-denominated and local currency hedged returns, while contagion was observed for local currency non-hedged returns. For the developed markets, the estimated coefficients for non-hedged returns during the global financial crisis indicate negative change of correlation and negative level of correlation (consistent with flight-to-quality), but the coefficients were not statistically significant. For the hedged returns, there has been a significant positive change of correlation but the correlation level was negative, indicating decoupling from the global stock market. The results for frontier markets were not statistically significant, confirming finding of Piljak and Swinkels (2017a) that frontier bond markets have lower level of market integration, which makes them less vulnerable to global crisis events.

\subsection{Portfolio management application}

What do the results above mean for an investor in global bond markets? In this section we focus on the returns in the extreme quantiles, and therefore analyze the worst weekly returns in the global aggregate bond market in more detail, and show the effect on a naively diversified portfolio of unhedged government bonds. The naïve portfolio is a portfolio with equal weights in the global developed government bond index, emerging markets local currency index, emerging markets USD-denominated bond index, and frontier markets index. Table 9 shows that this diversified portfolio ('DIV') had a return of an annualized $8.21 \%$ and an annualized volatility of $7.42 \%$, compared to an average return of $5.21 \%$ and volatility of $5.74 \%$ for the global aggregated bond market. Now we zoom in on the downside risk of investing in the global aggregate bond market by ranking each of the weeks of our sample based on the market return. The average return over the worst 10 weeks is $-2.21 \%$. While local currency emerging bond markets experienced a lower return during these weeks, on average $-3.22 \%$, frontier markets bonds did relatively well with a negative return of $-1.01 \%$. This is consistent with the low degree of dependence level of frontier markets, and higher average dependence of emerging markets local currency debt that we already saw in Table 2. While the diversified portfolio had a substantially higher volatility over the whole sample, the downside risk is on average the same as for the aggregate market index. ${ }^{22}$

During the global financial crisis, frontier markets did relatively poor during periods that the aggregate bond market did bad, with an average return of $-2.03 \%$. This is primarily due to an extreme negative return of $-11.59 \%$ in the week ending 10 October 2008 . Emerging markets bonds offset this huge negative week with a positive return of similar magnitude in the week ending 31 October 2008, but frontier markets did not. This positive correlation of frontier markets in the tail of the distribution of aggregate market returns is consistent with the significant increase in dependence that we reported in Table 3. During the Eurozone debt crisis, frontier markets hold out well, with an average return of $-0.58 \%$. Local currency emerging markets are relatively bad asset class during this period with $-1.90 \%$ return on average, and even a $-6.69 \%$ return during the week ending 7 May 2010 . The naïve diversified portfolio (again) shows slightly better returns than the aggregate market during the 10 worst weeks of this crisis period. This portfolio application shows that investors can use knowledge of the degree and structure of dependence between government bond markets to manage the downside risk of their government bond portfolios. As full portfolio optimization requires additional information on the required returns by investors and their attitudes for the entire risk profile, we leave that for further research. This section is an

\footnotetext{
22 The most extreme return is lower for the diversified portfolio, as the week ending 21 June 2013 had a negative return of -4.59\% versus the market $-2.69 \%$. The second largest drop in the aggregate market, ending 13 June 2008 , was $-2.68 \%$, whereas the diversified portfolio did relatively well with $-1.79 \%$.
} 
illustration of the practical use of the econometric techniques that we used earlier.

\section{Conclusions}

This paper studies the degree and structure of dependence in international government bond markets. Specifically, we focus on three categories of government bond markets (developed, emerging, and frontier) and analyze their dependence on the global bond market. We investigate whether the dependence structure is asymmetric (different upper and lower tail dependence) and affected by the 2007-2008 global financial crisis and Eurozone debt crisis, and what is the role of bond maturity. Furthermore, we also examine the currency aspect in the analysis of dependence structure by using: (i) returns converted to USD, (ii) returns hedged to USD using currency derivatives, and (iii) returns on USD-denominated government bonds.

The results lead to several conclusions. First, the study provides new evidence on asymmetric dependencies in international government bond markets. In particular, the dependence structure for emerging and frontier markets changes substantially due to the global financial crisis and Eurozone debt crisis, while developed markets exhibit a more stable dependence pattern. The results indicate that average dependence is not sufficient to describe a complete dependence structure in international government bond markets, meaning that the upper and lower tail dependence should also be considered as essential elements in measuring dependence. Second, regarding the currency aspect of the analysis, the results show that the degree of dependence varies substantially depending on whether local currency returns are hedged or unhedged. Third, degree of dependence and crisis-induced change in the dependence are increasing for longer maturities. Fourth, our finding that frontier markets exhibit a very low degree of dependence on the global bond market returns in both no-crisis and crisis times underscores the importance of their diversification potential for international investors in asset-allocation decisions. Fifth, results on regional and country analysis reveal that emerging and frontier markets cannot be treated as homogenous groups, and consequently regional and country aspect is important in analyzing the sensitivity of those markets to crises events. Finally, results of contagion analysis from global stock to bond markets during the global financial crisis confirm "equitylike" properties of emerging markets bonds.

In summary, the study has important practical implications for international investors in terms of asset allocation decision-making and the formulation of their investment strategies. Our application shows that investors can increase portfolio diversification in scenarios where the aggregate bond market experiences negative returns. By diversifying risk among emerging markets debt in both local and hard currencies, and frontier market bonds, downside risks are reduced. Our analyses indicate that there is not one specific asset class that always reduces fixed income portfolio risk, but rather that different asset classes may help during different crisis periods.

\section{Funding}

This work was supported by the Waldemar von Frenckells Foundation, Finland.

\section{Author Statement}

This statement certifies that all four authors have seen and approved the final version of the manuscript being submitted.

This statement confirms that article is the authors' original work, hasn't received prior publication and isn't under consideration for publication elsewhere.

\section{Declaration of Competing Interest}

The authors declare that they have no known competing financial interests or personal relationships that could have appeared to influence the work reported in this paper.

\section{Acknowledgements}

We would like to thank the anonymous referee, the editor (Jonathan Batten), Jyri Kinnunen, Dolly King, Ansgar Belke, Matthias Walting, and conference participants of the $17^{\text {th }}$ INFINITI Conference on International Finance, the $7^{\text {th }}$ Paris Financial Management Conference, the $59^{\text {th }}$ Annual Meeting of the Southern Finance Association (SFA), Research Seminar in Economics and Finance at the University of Jyväskylä, and the Summer Seminar of Finnish Economists for valuable comments. Vanja Piljak would like to thank the Waldemar von Frenckells Foundation from Finland for financial support.

\section{Appendix A. . Descriptive statistics for regions and countries}

See Table A1. 
Table A1

Descriptive statistics for regions and countries (emerging and frontier markets).

\begin{tabular}{|c|c|c|c|c|c|c|c|c|c|}
\hline & Mean & Median & Max & Min & St. Dev. & Skewness & Kurtosis & JB stat. & p-Value \\
\hline \multicolumn{10}{|c|}{ Panel A: Emerging Markets - Regions } \\
\hline Africa & 0.0013 & 0.0015 & 0.0670 & -0.0961 & 0.0105 & -1.2218 & 20.9846 & 11159.01 & 0.0000 \\
\hline Asia & 0.0017 & 0.0021 & 0.2278 & -0.2021 & 0.0169 & -1.2985 & 93.3055 & 276482.00 & 0.0000 \\
\hline Latin America & 0.0017 & 0.0026 & 0.1186 & -0.1476 & 0.0150 & -1.5210 & 24.2189 & 15565.37 & 0.0000 \\
\hline Europe & 0.0016 & 0.0020 & 0.1090 & -0.1360 & 0.0130 & -2.0748 & 35.0783 & 35441.23 & 0.0000 \\
\hline \multicolumn{10}{|c|}{ Panel B: Frontier Markets - Regions } \\
\hline Africa & 0.0016 & 0.0015 & 0.0704 & -0.0973 & 0.0120 & -1.5814 & 16.5893 & 6578.24 & 0.0000 \\
\hline Asia & 0.0017 & 0.0017 & 0.0713 & -0.1303 & 0.0136 & -2.4163 & 29.7860 & 21700.52 & 0.0000 \\
\hline Latin America & 0.0018 & 0.0029 & 0.1436 & -0.3571 & 0.0260 & -4.5275 & 59.2835 & 109816.90 & 0.0000 \\
\hline \multicolumn{10}{|c|}{ Panel C: Emerging markets - Individual Countries } \\
\hline Brazil & 0.0019 & 0.0024 & 0.1312 & -0.1285 & 0.0198 & -0.6628 & 17.3199 & 7005.93 & 0.0000 \\
\hline Colombia & 0.0017 & 0.0021 & 0.0911 & -0.1412 & 0.0140 & -1.6471 & 21.7161 & 12233.78 & 0.0000 \\
\hline Mexico & 0.0014 & 0.0017 & 0.1450 & -0.1276 & 0.0132 & -0.1532 & 34.8019 & 34262.98 & 0.0000 \\
\hline Panama & 0.0016 & 0.0016 & 0.0928 & -0.1489 & 0.0130 & -2.6862 & 39.0986 & 45120.55 & 0.0000 \\
\hline Peru & 0.0017 & 0.0020 & 0.0774 & -0.1407 & 0.0141 & -1.6335 & 20.2065 & 10390.77 & 0.0000 \\
\hline Philippines & 0.0017 & 0.0018 & 0.2177 & -0.2003 & 0.0160 & -1.0182 & 91.8186 & 267371.40 & 0.0000 \\
\hline Russia & 0.0016 & 0.0020 & 0.1033 & -0.1434 & 0.0137 & -1.9120 & 28.4420 & 22422.45 & 0.0000 \\
\hline South Africa & 0.0012 & 0.0015 & 0.0670 & -0.0961 & 0.0107 & -1.1627 & 19.3340 & 9209.66 & 0.0000 \\
\hline Turkey & 0.0017 & 0.0022 & 0.1226 & -0.1454 & 0.0165 & -1.8424 & 26.2460 & 18765.28 & 0.0000 \\
\hline \multicolumn{10}{|c|}{ Panel D: Frontier Markets - Individual Countries } \\
\hline Dominican R. & 0.0017 & 0.0016 & 0.1321 & -0.2297 & 0.0205 & -2.9245 & 36.1237 & 38231.43 & 0.0000 \\
\hline Ecuador & 0.0002 & 0.0017 & 0.2026 & -1.0841 & 0.0526 & -12.2173 & 235.9767 & 1854324.00 & 0.0000 \\
\hline Egypt & 0.0014 & 0.0014 & 0.0632 & -0.0754 & 0.0105 & -0.4293 & 12.7609 & 3220.41 & 0.0000 \\
\hline Pakistan & 0.0014 & 0.0014 & 0.1079 & -0.2707 & 0.0200 & -5.0270 & 72.8346 & 168213.40 & 0.0000 \\
\hline
\end{tabular}

This table reports descriptive statistics for USD-denominated weekly index returns for regions and countries of emerging and frontier markets from May 10, 2002 to December 29, 2017. A total number of

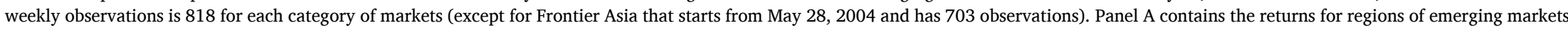

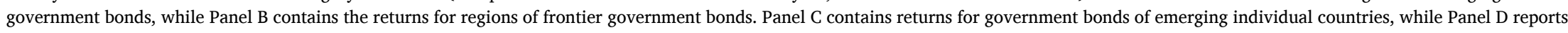
returns for government bonds of frontier individual countries. 


\section{Appendix B. . Regional analysis for emerging and frontier markets}

See Fig. B1 and Fig. B2.

See Table B1.
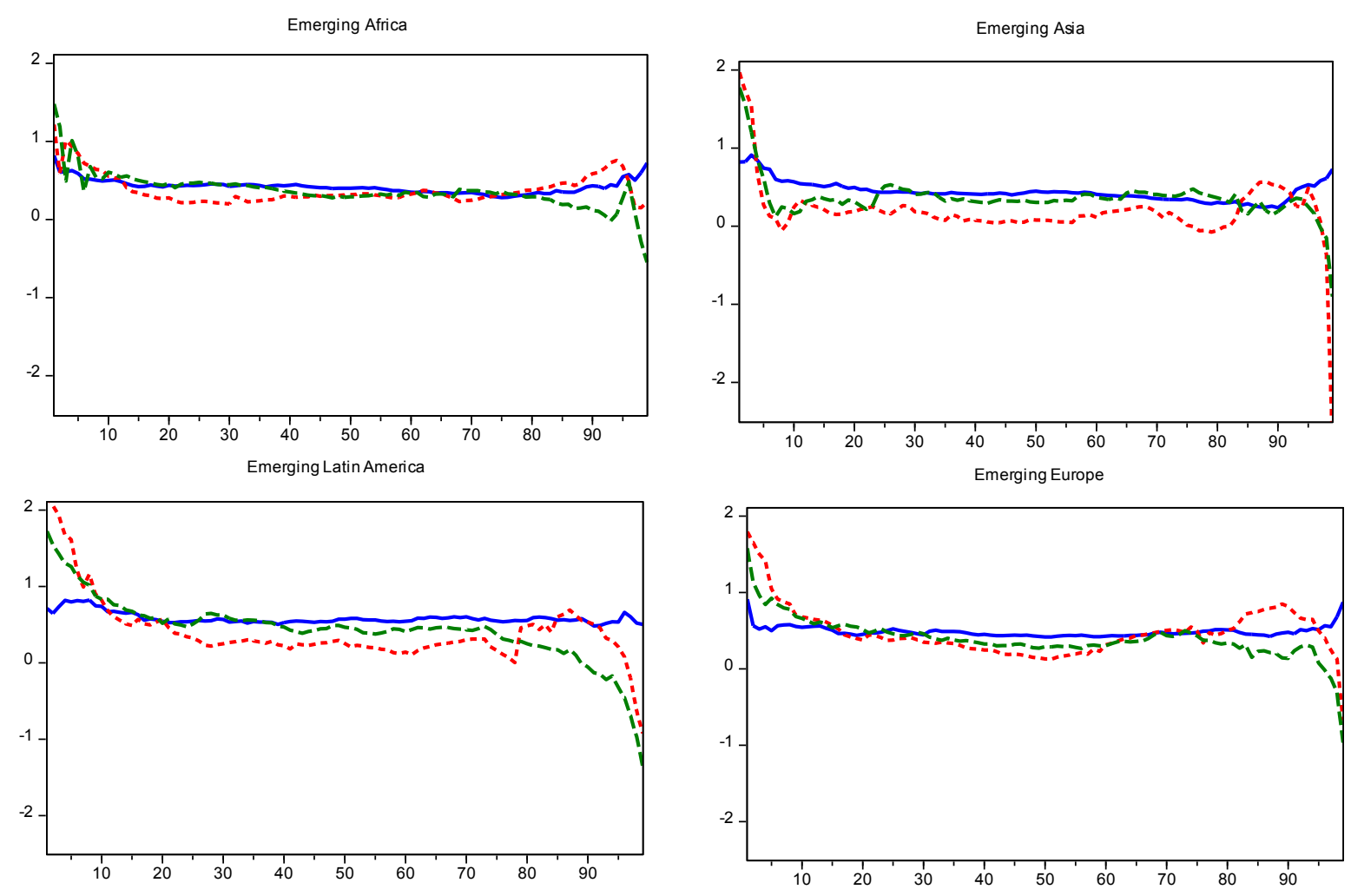

Not in crisis
- Dependence during global financial crisis
- Dependence during Eurozone debt crisis

Fig. B1. This figure shows the dependence structure of various emerging markets regions' USD-denominated bond returns on the returns of global bond market portfolio. 

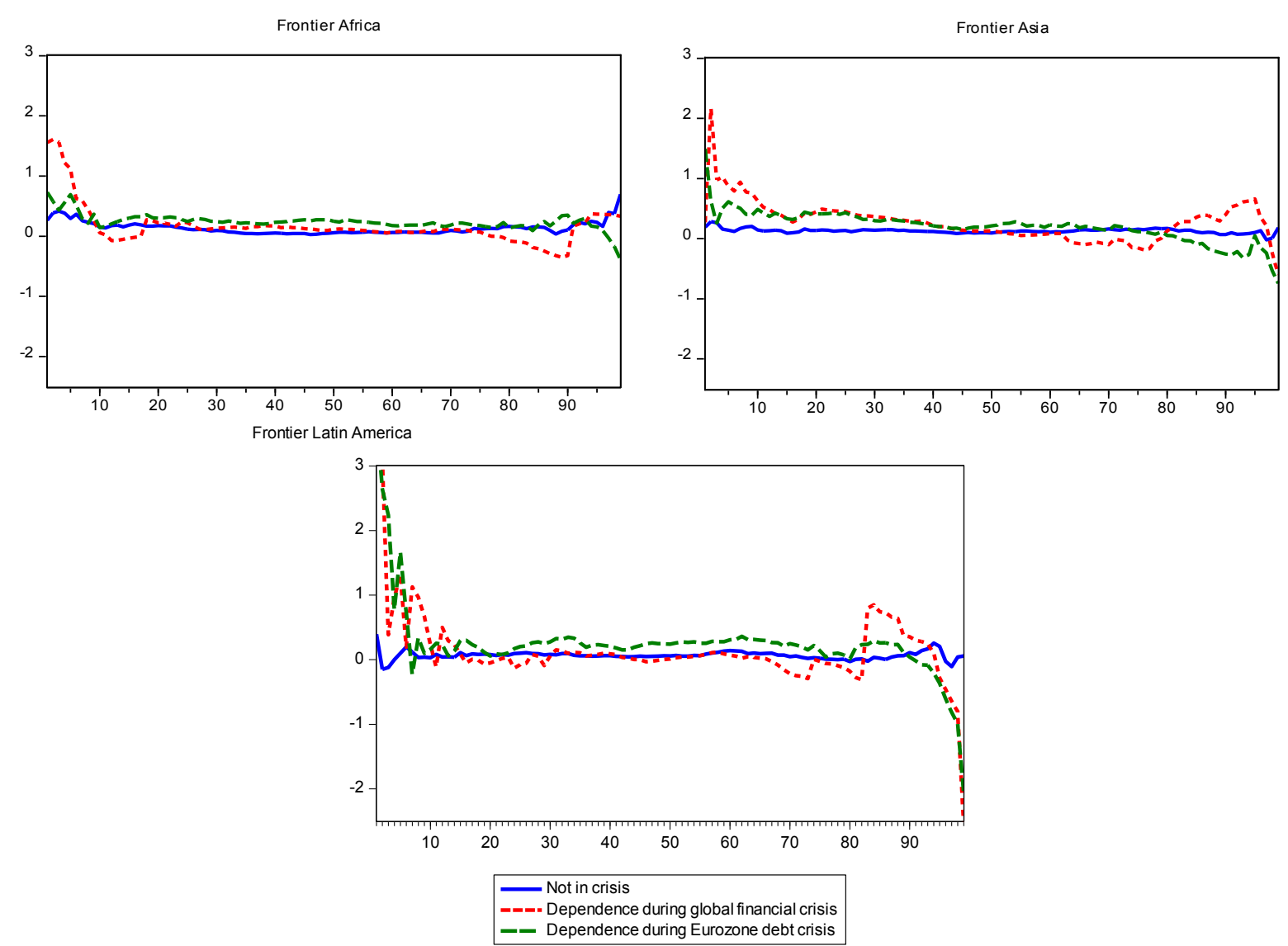

Fig. B2. This figure shows the dependence structure of various frontier markets regions' USD-denominated bond returns on the returns of global bond market portfolio. Due to data availability, the starting date for Asia region analysis is May 28, 2004. Middle East region is not included in the analysis due to issue of data availability (larger time breaks in observations).

Table B1

Degree of dependence for regions of emerging and frontier markets.

\begin{tabular}{llll}
\hline & $\beta$ & $\gamma_{1}$ Financial crisis & $\gamma_{2}$ Debt crisis \\
\hline Panel A: Emerging Markets (USD-denominated) & Not in crisis & & -0.056 \\
Africa & & -0.041 & -0.082 \\
Asia & 0.422 & -0.256 & -0.091 \\
Europe & 0.442 & -0.009 & -0.146 \\
Latin America & 0.486 & -0.75 & 0.103 \\
Panel B: Frontier Markets & 0.581 & & 0.071 \\
Africa & & 0.032 & 0.181 \\
Asia & 0.135 & 0.149 & \\
Latin America & 0.129 & 0.073 & \\
\hline
\end{tabular}

This table reports the average degree of dependence for regions of emerging and frontier markets (for USD-denominated bond returns). The degree of dependence is given by the average coefficient estimates across all quantiles according to the model defined by Equation (1). The table shows the average degree of dependence in the non-crisis period $(\beta)$, change in the degree of dependence due to the global financial crisis $\left(\gamma_{1}\right)$ and change in the degree of dependence due to the Eurozone debt crisis $\left(\gamma_{2}\right)$.

\section{Appendix C. . Country analysis for emerging and frontier markets}

See Table C1, Table C2, Table C3. 
Table C1

Structure and degree of the dependence.

\begin{tabular}{|c|c|c|c|c|c|c|c|c|c|c|c|c|}
\hline Quantile & 1 & 2 & 5 & 10 & 25 & 50 & 75 & 90 & 95 & 98 & 99 & Degree \\
\hline \multicolumn{13}{|c|}{ Panel A: Emerging Markets } \\
\hline Brazil & $\begin{array}{l}0.399^{* * * *} \\
(2.52)\end{array}$ & $\begin{array}{l}0.799^{* * * *} \\
(8.05)\end{array}$ & $\begin{array}{l}0.786^{* * *} \\
(5.85)\end{array}$ & $\begin{array}{l}0.851^{* * *} \\
(7.10)\end{array}$ & $\begin{array}{l}0.654^{* * * *} \\
(6.84)\end{array}$ & $\begin{array}{l}0.599 * * * \\
(6.77)\end{array}$ & $\begin{array}{l}0.661 * * * \\
(5.88)\end{array}$ & $\begin{array}{l}0.646^{* * *} \\
(6.50)\end{array}$ & $\begin{array}{l}0.647^{* * * *} \\
(5.55)\end{array}$ & $\begin{array}{l}0.746^{* * * *} \\
(4.76)\end{array}$ & $\begin{array}{l}0.819^{* * * *} \\
(6.80)\end{array}$ & 0.83 \\
\hline Colombia & $\begin{array}{l}0.835^{* * *} \\
(6.87)\end{array}$ & $\begin{array}{l}0.885^{* * *} \\
(12.41)\end{array}$ & $\begin{array}{l}0.911^{* * *} \\
(15.02)\end{array}$ & $\begin{array}{l}0.807^{* * * *} \\
(13.27)\end{array}$ & $\begin{array}{l}0.553^{* * *} \\
(6.58)\end{array}$ & $\begin{array}{l}0.505^{* * *} \\
(7.35)\end{array}$ & $\begin{array}{l}0.452^{* * *} \\
(5.83)\end{array}$ & $\begin{array}{l}0.577^{* * *} \\
(6.58)\end{array}$ & $\begin{array}{l}0.788^{* * *} \\
(7.41)\end{array}$ & $\begin{array}{l}0.854^{* * *} \\
(3.24)\end{array}$ & $\begin{array}{l}0.688^{* * * *} \\
(3.57)\end{array}$ & 0.57 \\
\hline Mexico & $\begin{array}{l}1.276^{* * * *} \\
(8.88)\end{array}$ & $\begin{array}{l}0.987^{* * * *} \\
(7.40)\end{array}$ & $\begin{array}{l}0.912^{* * *} \\
(11.23)\end{array}$ & $\begin{array}{l}0.767^{* * * *} \\
(10.11)\end{array}$ & $\begin{array}{l}0.613^{* * *} \\
(9.02)\end{array}$ & $\begin{array}{l}0.561^{* * * *} \\
(8.49)\end{array}$ & $\begin{array}{l}0.527 * * * * \\
(7.70)\end{array}$ & $\begin{array}{l}0.573^{* * *} \\
(8.26)\end{array}$ & $\begin{array}{l}0.572^{* * *} \\
(5.05)\end{array}$ & $\begin{array}{l}0.575^{* * *} \\
(3.00)\end{array}$ & $\begin{array}{l}1.069^{* * * *} \\
(8.05)\end{array}$ & 0.45 \\
\hline Panama & $\begin{array}{l}0.708^{* * * *} \\
(8.48)\end{array}$ & $\begin{array}{l}0.701^{* * *} \\
(10.78)\end{array}$ & $\begin{array}{l}0.665^{* * * *} \\
(8.18)\end{array}$ & $\begin{array}{l}0.605^{* * * *} \\
(10.11)\end{array}$ & $\begin{array}{l}0.392^{* * *} \\
(6.48)\end{array}$ & $\begin{array}{l}0.383^{* * *} \\
(6.44)\end{array}$ & $\begin{array}{l}0.381^{* * *} \\
(6.23)\end{array}$ & $\begin{array}{l}0.411^{* * *} \\
(3.77)\end{array}$ & $\begin{array}{l}0.493^{* * * *} \\
(3.55)\end{array}$ & $\begin{array}{l}0.488^{* * *} \\
(3.08)\end{array}$ & $\begin{array}{l}0.631^{* * *} \\
(6.13)\end{array}$ & 0.42 \\
\hline Peru & $\begin{array}{l}0.728^{* * * *} \\
(4.86)\end{array}$ & $\begin{array}{l}0.961^{* * *} \\
(8.21)\end{array}$ & $\begin{array}{l}0.743^{* * *} \\
(8.49)\end{array}$ & $\begin{array}{l}0.572^{\text {*** }} \\
(3.60)\end{array}$ & $\begin{array}{l}0.421 * * * \\
(5.19)\end{array}$ & $\begin{array}{l}0.419^{* * * *} \\
(5.79)\end{array}$ & $\begin{array}{l}0.474^{* * * *} \\
(5.99)\end{array}$ & $\begin{array}{l}0.449^{* * * *} \\
(4.36)\end{array}$ & $\begin{array}{l}0.560^{* * *} \\
(5.37)\end{array}$ & $\begin{array}{l}0.993^{* * * *} \\
(4.90)\end{array}$ & $\begin{array}{l}1.170^{* * *} \\
(8.22)\end{array}$ & 0.40 \\
\hline Phillipines & $\begin{array}{l}0.973^{* * *} \\
(13.19)\end{array}$ & $\begin{array}{l}0.799^{* * *} \\
(12.25)\end{array}$ & $\begin{array}{l}0.743^{* * *} \\
(8.85)\end{array}$ & $\begin{array}{l}0.567^{* * * *} \\
(6.79)\end{array}$ & $\begin{array}{l}0.437^{* * * *} \\
(8.56)\end{array}$ & $\begin{array}{l}0.353^{* * *} \\
(6.50)\end{array}$ & $\begin{array}{l}0.298^{* * *} \\
(5.42)\end{array}$ & $\begin{array}{l}0.302^{* * *} \\
(2.79)\end{array}$ & $\begin{array}{l}0.453^{* * * *} \\
(3.18)\end{array}$ & $\begin{array}{l}0.608^{* * *} \\
(3.98)\end{array}$ & $\begin{array}{l}0.419^{* * *} \\
(4.19)\end{array}$ & 0.38 \\
\hline Russia & $\begin{array}{c}0.320 \\
(1.44)\end{array}$ & $\begin{array}{l}0.794^{* * * *} \\
(3.28)\end{array}$ & $\begin{array}{l}0.626^{* * * *} \\
(5.54)\end{array}$ & $\begin{array}{l}0.544^{* * * *} \\
(7.10)\end{array}$ & $\begin{array}{l}0.411^{* * * *} \\
(8.05)\end{array}$ & $\begin{array}{l}0.388^{* * * *} \\
(8.09)\end{array}$ & $\begin{array}{l}0.442^{* * * *} \\
(7.91)\end{array}$ & $\begin{array}{l}0.478^{* * * *} \\
(5.21)\end{array}$ & $\begin{array}{l}0.520^{* * * *} \\
(4.37)\end{array}$ & $\begin{array}{c}0.208 \\
(1.47)\end{array}$ & $\begin{array}{c}0.429 \\
(1.09)\end{array}$ & 0.35 \\
\hline South Africa & $\begin{array}{l}0.823^{* * * *} \\
(5.69)\end{array}$ & $\begin{array}{l}0.729^{* * *} \\
(5.40)\end{array}$ & $\begin{array}{l}0.664^{* * *} \\
(10.67)\end{array}$ & $\begin{array}{l}0.558^{* * *} \\
(8.63)\end{array}$ & $\begin{array}{l}0.498^{* * * *} \\
(11.09)\end{array}$ & $\begin{array}{l}0.478^{* * *} \\
(10.36)\end{array}$ & $\begin{array}{l}0.421^{* * * *} \\
(6.09)\end{array}$ & $\begin{array}{l}0.470^{* * *} \\
(7.11)\end{array}$ & $\begin{array}{l}0.597^{* * * *} \\
(4.38)\end{array}$ & $\begin{array}{l}0.600^{* * * *} \\
(5.42)\end{array}$ & $\begin{array}{l}0.679^{* * *} \\
(5.57)\end{array}$ & 0.32 \\
\hline Turkey & $\begin{array}{l}1.482^{* * * *} \\
(7.69)\end{array}$ & $\begin{array}{l}0.981^{* * *} \\
(3.82)\end{array}$ & $\begin{array}{l}0.569^{* * *} \\
(7.09)\end{array}$ & $\begin{array}{l}0.608^{* * *} \\
(8.74)\end{array}$ & $\begin{array}{l}0.510^{* * *} \\
(5.03)\end{array}$ & $\begin{array}{l}0.421^{* * *} \\
(5.27)\end{array}$ & $\begin{array}{l}0.515^{* * * *} \\
(7.11)\end{array}$ & $\begin{array}{l}0.561^{* * *} \\
(5.51)\end{array}$ & $\begin{array}{l}0.731^{* * *} \\
(7.60)\end{array}$ & $\begin{array}{l}0.872^{* * *} \\
(4.99)\end{array}$ & $\begin{array}{l}1.050^{* * *} \\
(6.07)\end{array}$ & 0.30 \\
\hline \multicolumn{13}{|c|}{ Panel B: Frontier Markets } \\
\hline Dominican Republic & $\begin{array}{l}-0.590 \\
(-1.40)\end{array}$ & $\begin{array}{l}-0.184 \\
(-0.51)\end{array}$ & $\begin{array}{l}0.337^{* * * *} \\
(2.68)\end{array}$ & $\begin{array}{l}0.314^{* * * *} \\
(2.74)\end{array}$ & $\begin{array}{c}0.128 \\
(1.47)\end{array}$ & $\begin{array}{c}0.058 \\
(0.99)\end{array}$ & $\begin{array}{l}0.232^{* *} \\
(2.46)\end{array}$ & $\begin{array}{l}0.233^{*} \\
(1.81)\end{array}$ & $\begin{array}{l}0.326^{*} \\
(1.80)\end{array}$ & $\begin{array}{l}0.545^{* *} \\
(1.99)\end{array}$ & $\begin{array}{r}0.347 \\
(0.59)\end{array}$ & 0.58 \\
\hline Ecuador & $\begin{array}{l}0.622^{* * *} \\
(3.14)\end{array}$ & $\begin{array}{l}0.566^{* * *} \\
(2.61)\end{array}$ & $\begin{array}{l}0.447^{* *} \\
(2.41)\end{array}$ & $\begin{array}{l}0.322^{* *} \\
(2.29)\end{array}$ & $\begin{array}{c}0.106 \\
(1.29)\end{array}$ & $\begin{array}{c}0.083 \\
(1.04)\end{array}$ & $\begin{array}{l}0.413^{* * *} \\
(4.31)\end{array}$ & $\begin{array}{l}0.454^{* * *} \\
(3.02)\end{array}$ & $\begin{array}{l}0.384^{*} \\
(1.74)\end{array}$ & $\begin{array}{l}0.776^{\text {**** }} \\
(3.04)\end{array}$ & $\begin{array}{l}1.436^{* * * *} \\
(4.58)\end{array}$ & 0.53 \\
\hline Egypt & $\begin{array}{c}-0.267 \\
(-0.51)\end{array}$ & $\begin{array}{c}0.195 \\
(0.08)\end{array}$ & $\begin{array}{l}0.413^{* * * *} \\
(3.73)\end{array}$ & $\begin{array}{l}0.319^{* * *} \\
(5.05)\end{array}$ & $\begin{array}{l}0.248^{* * *} \\
(5.55)\end{array}$ & $\begin{array}{l}0.172^{* * *} \\
(3.89)\end{array}$ & $\begin{array}{l}0.220^{* * * *} \\
(4.94)\end{array}$ & $\begin{array}{l}0.446^{* * * *} \\
(6.08)\end{array}$ & $\begin{array}{l}0.643^{* * *} \\
(5.60)\end{array}$ & $\begin{array}{l}0.704^{* *} \\
(2.56)\end{array}$ & $\begin{array}{l}0.510^{* * *} \\
(2.81)\end{array}$ & 0.42 \\
\hline Pakistan & $\begin{array}{l}-1.399^{* * *} \\
(-3.66)\end{array}$ & $\begin{array}{l}-0.455 \\
(-0.50)\end{array}$ & $\begin{array}{l}0.305^{* *} \\
(2.51)\end{array}$ & $\begin{array}{l}0.281^{* * *} \\
(3.05)\end{array}$ & $\begin{array}{l}0.097^{* *} \\
(2.55)\end{array}$ & $\begin{array}{c}0.025 \\
(1.10)\end{array}$ & $\begin{array}{l}0.188^{* * * *} \\
(3.74)\end{array}$ & $\begin{array}{c}0.132 \\
(0.70)\end{array}$ & $\begin{array}{c}0.070 \\
(0.38)\end{array}$ & $\begin{array}{c}0.189 \\
(1.46)\end{array}$ & $\begin{array}{l}0.427^{* * *} \\
(3.69)\end{array}$ & 0.28 \\
\hline
\end{tabular}

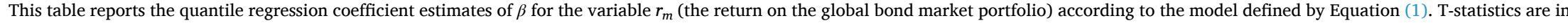
parentheses. The last column shows degree of dependence. 
Table C2

Structure and degree of the dependence (change during the global financial crisis period).

\begin{tabular}{|c|c|c|c|c|c|c|c|c|c|c|c|c|}
\hline Quantile & 1 & 2 & 5 & 10 & 25 & 50 & 75 & 90 & 95 & 98 & 99 & Degree \\
\hline \multicolumn{13}{|c|}{ Panel A: Emerging Markets } \\
\hline Brazil & $\begin{array}{l}3.377^{* * * *} \\
(4.34)\end{array}$ & $\begin{array}{l}1.853^{* * *} \\
(4.83)\end{array}$ & $\begin{array}{l}1.259^{* * *} \\
(3.10)\end{array}$ & $\begin{array}{l}0.594^{* *} \\
(2.05)\end{array}$ & $\begin{array}{l}-0.320^{*} \\
(-1.75)\end{array}$ & $\begin{array}{l}-0.449 \text { *** } \\
(-2.70)\end{array}$ & $\begin{array}{l}-0.455 \\
(-0.76)\end{array}$ & $\begin{array}{l}-0.211 \\
(-0.39)\end{array}$ & $\begin{array}{l}-1.251^{* * *} \\
(-5.93)\end{array}$ & $\begin{array}{l}-1.837^{* * *} \\
(-3.85)\end{array}$ & $\begin{array}{l}-2.264^{* * * *} \\
(-3.82)\end{array}$ & -0.24 \\
\hline Colombia & $\begin{array}{l}1.378^{* * *} \\
(8.64)\end{array}$ & $\begin{array}{l}1.039 * * * \\
(5.25)\end{array}$ & $\begin{array}{l}0.507^{* * * *} \\
(3.37)\end{array}$ & $\begin{array}{c}0.006 \\
(0.02)\end{array}$ & $\begin{array}{l}-0.245^{*} \\
(-1.72)\end{array}$ & $\begin{array}{l}-0.303^{*} \\
(-1.67)\end{array}$ & $\begin{array}{l}-0.061 \\
(-0.20)\end{array}$ & $\begin{array}{l}0.070 \\
(0.40)\end{array}$ & $\begin{array}{l}-0.439 * * \\
(-2.04)\end{array}$ & $\begin{array}{l}-1.098 \\
(-0.50)\end{array}$ & $\begin{array}{l}-1.178^{* * *} \\
(-5.71)\end{array}$ & -0.17 \\
\hline Mexico & $\begin{array}{l}1.045^{* * *} \\
(9.25)\end{array}$ & $\begin{array}{l}1.019 \\
(1.58)\end{array}$ & $\begin{array}{c}0.248 \\
(0.55)\end{array}$ & $\begin{array}{c}0.232 \\
(0.92)\end{array}$ & $\begin{array}{l}-0.126 \\
(-1.09)\end{array}$ & $\begin{array}{l}-0.140 \\
(-0.95)\end{array}$ & $\begin{array}{l}0.355^{* *} \\
(2.14)\end{array}$ & $\begin{array}{l}0.126 \\
(0.44)\end{array}$ & $\begin{array}{l}0.289 \\
(1.31)\end{array}$ & $\begin{array}{l}-0.029 \\
(-0.09)\end{array}$ & $\begin{array}{l}-2.193^{* * *} \\
(-11.30)\end{array}$ & 0.07 \\
\hline Panama & $\begin{array}{l}1.407^{* * * *} \\
(9.56)\end{array}$ & $\begin{array}{l}1.201^{* * *} \\
(7.81)\end{array}$ & $\begin{array}{c}0.365 \\
(0.74)\end{array}$ & $\begin{array}{l}-0.345 \\
(-0.49)\end{array}$ & $\begin{array}{l}-0.409^{* * *} \\
(-2.27)\end{array}$ & $\begin{array}{l}-0.277^{* * *} \\
(-2.69)\end{array}$ & $\begin{array}{l}-0.087 \\
(-0.56)\end{array}$ & $\begin{array}{l}0.037 \\
(0.19)\end{array}$ & $\begin{array}{l}0.230 \\
(1.32)\end{array}$ & $\begin{array}{l}-0.305 \\
(-0.27)\end{array}$ & $\begin{array}{l}-0.756^{* * *} \\
(-5.33)\end{array}$ & -0.15 \\
\hline Peru & $\begin{array}{l}1.624^{* * * *} \\
(6.85)\end{array}$ & $\begin{array}{l}1.116^{* * * *} \\
(5.81)\end{array}$ & $\begin{array}{c}0.381 \\
(1.09)\end{array}$ & $\begin{array}{c}-0.030 \\
(-0.07)\end{array}$ & $\begin{array}{l}-0.182 \\
(-1.09)\end{array}$ & $\begin{array}{l}-0.047 \\
(-0.26)\end{array}$ & $\begin{array}{l}0.161 \\
(1.03)\end{array}$ & $\begin{array}{l}-0.286 \\
(-1.17)\end{array}$ & $\begin{array}{l}-0.592^{* *} \\
(-2.14)\end{array}$ & $\begin{array}{l}-1.149^{* * *} \\
(-3.33)\end{array}$ & $\begin{array}{l}-1.341^{* * *} \\
(-4.77)\end{array}$ & -0.05 \\
\hline Phillipines & $\begin{array}{l}0.798^{*} \\
(1.92)\end{array}$ & $\begin{array}{c}0.580 \\
(0.90)\end{array}$ & $\begin{array}{l}-0.381 \\
(-0.32)\end{array}$ & $\begin{array}{l}-0.449^{* * *} \\
(-2.70)\end{array}$ & $\begin{array}{l}-0.285 \\
(-1.16)\end{array}$ & $\begin{array}{l}-0.307^{* *} \\
(-2.27)\end{array}$ & $\begin{array}{l}-0.281 \\
(-0.93)\end{array}$ & $\begin{array}{l}0.113 \\
(0.60)\end{array}$ & $\begin{array}{l}-0.165 \\
(-0.27)\end{array}$ & $\begin{array}{l}-1.049^{*} \\
(-1.90)\end{array}$ & $\begin{array}{l}-3.005^{* * *} \\
(-17.73)\end{array}$ & -0.27 \\
\hline Russia & $\begin{array}{l}1.880^{* * *} \\
(6.06)\end{array}$ & $\begin{array}{l}1.262^{* * * *} \\
(5.52)\end{array}$ & $\begin{array}{l}0.850^{* * * *} \\
(2.64)\end{array}$ & $\begin{array}{l}0.549 * * \\
(2.49)\end{array}$ & $\begin{array}{l}0.032 \\
(0.25)\end{array}$ & $\begin{array}{l}-0.085 \\
(-0.99)\end{array}$ & $\begin{array}{l}0.062 \\
(0.59)\end{array}$ & $\begin{array}{l}-0.400 \\
(-1.49)\end{array}$ & $\begin{array}{l}-0.727^{* * *} \\
(-3.73)\end{array}$ & $\begin{array}{l}-1.207^{* * *} \\
(-7.32)\end{array}$ & $\begin{array}{l}-1.469^{* * *} \\
(-3.46)\end{array}$ & 0.00 \\
\hline South Africa & $\begin{array}{c}0.424 \\
(0.97)\end{array}$ & $\begin{array}{r}0.087 \\
(0.02)\end{array}$ & $\begin{array}{l}0.219^{*} \\
(1.91)\end{array}$ & $\begin{array}{r}0.157 \\
(0.85)\end{array}$ & $\begin{array}{l}-0.172 \\
(-1.24)\end{array}$ & $\begin{array}{l}-0.158 \\
(-1.63)\end{array}$ & $\begin{array}{l}-0.139 \\
(-0.60)\end{array}$ & $\begin{array}{l}0.064 \\
(0.26)\end{array}$ & $\begin{array}{l}0.085 \\
(0.50)\end{array}$ & $\begin{array}{l}-0.451 \\
(-1.60)\end{array}$ & $\begin{array}{l}-0.460^{*} \\
(-1.85)\end{array}$ & -0.09 \\
\hline Turkey & $\begin{array}{l}0.505^{* * *} \\
(2.66)\end{array}$ & $\begin{array}{l}0.734^{* * * *} \\
(3.15)\end{array}$ & $\begin{array}{c}0.541 \\
(1.59)\end{array}$ & $\begin{array}{l}-0.090 \\
(-0.37)\end{array}$ & $\begin{array}{l}-0.253 \\
(-1.20)\end{array}$ & $\begin{array}{l}-0.413^{* *} \\
(-2.13)\end{array}$ & $\begin{array}{l}-0.202 \\
(-0.44)\end{array}$ & $\begin{array}{l}0.202 \\
(0.47)\end{array}$ & $\begin{array}{l}0.373^{* * *} \\
(2.52)\end{array}$ & $\begin{array}{l}-0.270 \\
(-0.21)\end{array}$ & $\begin{array}{l}-1.373^{* * *} \\
(-6.07)\end{array}$ & -0.14 \\
\hline Panel B: Frontier Mark & & & & & & & & & & & & \\
\hline Dominican Republic & $\begin{array}{l}4.084^{* * *} \\
(4.71)\end{array}$ & $\begin{array}{l}2.935^{* * *} \\
(4.19)\end{array}$ & $\begin{array}{r}1.167 \\
(1.45)\end{array}$ & $\begin{array}{c}0.679 \\
(1.58)\end{array}$ & $\begin{array}{l}0.156 \\
(1.00)\end{array}$ & $\begin{array}{l}-0.062 \\
(-0.58)\end{array}$ & $\begin{array}{l}-0.477^{* *} \\
(-2.50)\end{array}$ & $\begin{array}{l}0.025 \\
(0.06)\end{array}$ & $\begin{array}{l}-0.917 \\
(-0.92)\end{array}$ & $\begin{array}{l}-2.008^{* * *} \\
(-5.28)\end{array}$ & $\begin{array}{l}-1.844^{* * *} \\
(-2.49)\end{array}$ & -0.06 \\
\hline Ecuador & $\begin{array}{l}-3.169^{* * *} \\
(-4.03)\end{array}$ & $\begin{array}{l}-2.741^{* * *} \\
(-3.94)\end{array}$ & $\begin{array}{l}-3.068^{* * *} \\
(-4.46)\end{array}$ & $\begin{array}{c}-0.644 \\
(-1.00)\end{array}$ & $\begin{array}{l}0.054 \\
(0.19)\end{array}$ & $\begin{array}{l}-0.139 \\
(-0.75)\end{array}$ & $\begin{array}{l}0.016 \\
(0.03)\end{array}$ & $\begin{array}{l}0.908 \\
(1.14)\end{array}$ & $\begin{array}{l}1.902^{* *} \\
(2.50)\end{array}$ & $\begin{array}{l}-2.335 \\
(-0.41)\end{array}$ & $\begin{array}{l}-5.563^{* * *} \\
(-6.06)\end{array}$ & -0.17 \\
\hline Egypt & $\begin{array}{l}1.321^{* *} \\
(2.22)\end{array}$ & $\begin{array}{r}0.407 \\
(0.17)\end{array}$ & $\begin{array}{l}-0.164 \\
(-0.96)\end{array}$ & $\begin{array}{l}-0.189 \\
(-0.63)\end{array}$ & $\begin{array}{l}-0.222 \\
(-1.00)\end{array}$ & $\begin{array}{l}-0.162^{* *} \\
(-2.03)\end{array}$ & $\begin{array}{l}-0.201^{*} \\
(-1.73)\end{array}$ & $\begin{array}{l}-0.500^{* * *} \\
(-4.23)\end{array}$ & $\begin{array}{l}-0.956 * * * * \\
(-6.14)\end{array}$ & $\begin{array}{l}-1.111^{* * *} \\
(-3.95)\end{array}$ & $\begin{array}{l}-0.926^{* * *} \\
(-4.90)\end{array}$ & -0.24 \\
\hline Pakistan & $\begin{array}{c}0.295 \\
(0.27)\end{array}$ & $\begin{array}{c}0.029 \\
(0.03)\end{array}$ & $\begin{array}{c}0.063 \\
(0.17)\end{array}$ & $\begin{array}{l}-0.248 \\
(-0.55)\end{array}$ & $\begin{array}{l}-0.262 \\
(-1.38)\end{array}$ & $\begin{array}{l}-0.034 \\
(-0.36)\end{array}$ & $\begin{array}{l}-0.171 \\
(-0.44)\end{array}$ & $\begin{array}{l}-0.062 \\
(-0.16)\end{array}$ & $\begin{array}{l}-0.626^{*} \\
(-1.92)\end{array}$ & $\begin{array}{l}-1.663^{* * *} \\
(-3.52)\end{array}$ & $\begin{array}{l}-2.059^{* * *} \\
(-4.84)\end{array}$ & -0.21 \\
\hline
\end{tabular}

This table reports the quantile regression coefficient estimates of $\gamma_{1}$ (change of the dependence during the global financial crisis period) according to the model defined by Equation (1). T-statistics are in parentheses. The last column shows change in degree of dependence. 
Table C3

Structure and degree of the dependence (change during the Eurozone debt crisis period).

\begin{tabular}{|c|c|c|c|c|c|c|c|c|c|c|c|c|}
\hline Quantile & 1 & 2 & 5 & 10 & 25 & 50 & 75 & 90 & 95 & 98 & 99 & Degree \\
\hline \multicolumn{13}{|c|}{ Panel A: Emerging Markets } \\
\hline Brazil & $\begin{array}{l}2.200^{* * *} \\
(3.54)\end{array}$ & $\begin{array}{l}1.129^{* * *} \\
(2.11)\end{array}$ & $\begin{array}{c}0.683 \\
(1.15)\end{array}$ & $\begin{array}{c}0.506 \\
(1.13)\end{array}$ & $\begin{array}{l}-0.128 \\
(-0.79)\end{array}$ & $\begin{array}{l}-0.167 \\
(-1.29)\end{array}$ & $\begin{array}{l}-0.475 * * \\
(-2.39)\end{array}$ & $\begin{array}{l}-1.023^{* * *} \\
(-3.48)\end{array}$ & $\begin{array}{l}-1.661^{* * * *} \\
(-2.56)\end{array}$ & $\begin{array}{l}-2.345^{* * * *} \\
(-7.23)\end{array}$ & $\begin{array}{l}-2.604^{* k * k} \\
(-8.49)\end{array}$ & -0.30 \\
\hline Colombia & $\begin{array}{l}0.935^{* * *} \\
(3.50)\end{array}$ & $\begin{array}{l}0.572^{*} \\
(1.84)\end{array}$ & $\begin{array}{c}0.215 \\
(0.65)\end{array}$ & $\begin{array}{c}0.016 \\
(0.06)\end{array}$ & $\begin{array}{l}-0.015 \\
(-0.09)\end{array}$ & $\begin{array}{l}0.042 \\
(0.33)\end{array}$ & $\begin{array}{l}-0.031 \\
(-0.14)\end{array}$ & $\begin{array}{l}-0.530^{* *} \\
(-2.23)\end{array}$ & $\begin{array}{l}-0.652^{* *} \\
(-2.52)\end{array}$ & $\begin{array}{l}-1.118 \\
(-1.25)\end{array}$ & $\begin{array}{l}-1.168^{* * *} \\
(-2.69)\end{array}$ & -0.09 \\
\hline Mexico & $\begin{array}{l}0.469 * * \\
(2.25)\end{array}$ & $\begin{array}{c}0.386 \\
(0.94)\end{array}$ & $\begin{array}{c}0.045 \\
(0.19)\end{array}$ & $\begin{array}{l}-0.046 \\
(-0.28)\end{array}$ & $\begin{array}{l}-0.150 \\
(-1.34)\end{array}$ & $\begin{array}{l}-0.053 \\
(-0.37)\end{array}$ & $\begin{array}{l}0.032 \\
(0.19)\end{array}$ & $\begin{array}{l}-0.342^{* *} \\
(-2.28)\end{array}$ & $\begin{array}{l}-0.331 \\
(-1.26)\end{array}$ & $\begin{array}{l}-0.733^{* *} \\
(-2.00)\end{array}$ & $\begin{array}{l}-2.249^{* * * *} \\
(-7.23)\end{array}$ & -0.14 \\
\hline Panama & $\begin{array}{l}0.945^{* * * *} \\
(4.81)\end{array}$ & $\begin{array}{l}0.722^{* * * *} \\
(3.28)\end{array}$ & $\begin{array}{c}0.273 \\
(1.35)\end{array}$ & $\begin{array}{c}0.002 \\
(0.01)\end{array}$ & $\begin{array}{l}0.026 \\
(0.16)\end{array}$ & $\begin{array}{l}-0.235 \\
(-1.49)\end{array}$ & $\begin{array}{l}-0.154 \\
(-1.31)\end{array}$ & $\begin{array}{l}-0.420^{* * *} \\
(-3.03)\end{array}$ & $\begin{array}{l}-0.175 \\
(-0.72)\end{array}$ & $\begin{array}{l}-1.240^{*} \\
(-1.76)\end{array}$ & $\begin{array}{l}0.278 \\
(0.74)\end{array}$ & -0.14 \\
\hline Peru & $\begin{array}{c}0.330 \\
(0.22)\end{array}$ & $\begin{array}{l}-0.531 \\
(-1.27)\end{array}$ & $\begin{array}{l}0.322^{* *} \\
(2.06)\end{array}$ & $\begin{array}{c}0.248 \\
(1.31)\end{array}$ & $\begin{array}{l}0.053 \\
(0.26)\end{array}$ & $\begin{array}{l}0.208 \\
(1.29)\end{array}$ & $\begin{array}{l}-0.042 \\
(-0.22)\end{array}$ & $\begin{array}{l}-0.268 \\
(-1.48)\end{array}$ & $\begin{array}{l}-0.356 \\
(-1.51)\end{array}$ & $\begin{array}{l}-1.516^{* * *} \\
(-3.54)\end{array}$ & $\begin{array}{l}-0.594 \\
(-0.20)\end{array}$ & -0.02 \\
\hline Phillipines & $\begin{array}{l}0.702^{* *} \\
(2.03)\end{array}$ & $\begin{array}{c}0.591 \\
(1.54)\end{array}$ & $\begin{array}{l}-0.154 \\
(-0.28)\end{array}$ & $\begin{array}{l}-0.421 \\
(-1.53)\end{array}$ & $\begin{array}{l}-0.061 \\
(-0.32)\end{array}$ & $\begin{array}{l}-0.062 \\
(-0.42)\end{array}$ & $\begin{array}{l}0.154 \\
(1.42)\end{array}$ & $\begin{array}{l}-0.158 \\
(-0.86)\end{array}$ & $\begin{array}{l}-0.255 \\
(-0.41)\end{array}$ & $\begin{array}{l}-0.865 \\
(-1.23)\end{array}$ & $\begin{array}{l}-1.227 \\
(-1.60)\end{array}$ & -0.06 \\
\hline Russia & $\begin{array}{l}1.595^{* * *} \\
(4.14)\end{array}$ & $\begin{array}{l}0.861^{* * * *} \\
(3.21)\end{array}$ & $\begin{array}{l}0.516^{* * *} \\
(1.97)\end{array}$ & $\begin{array}{c}0.274 \\
(1.26)\end{array}$ & $\begin{array}{l}-0.028 \\
(-0.24)\end{array}$ & $\begin{array}{l}-0.143 \\
(-1.33)\end{array}$ & $\begin{array}{l}-0.081 \\
(-0.57)\end{array}$ & $\begin{array}{l}-0.274 \\
(-0.81)\end{array}$ & $\begin{array}{l}-0.626^{*} \\
(-1.86)\end{array}$ & $\begin{array}{l}-1.118^{* * *} \\
(-3.99)\end{array}$ & $\begin{array}{l}-1.629 * * * \\
(-2.80)\end{array}$ & -0.07 \\
\hline South Africa & $\begin{array}{l}0.667^{* * *} \\
(3.95)\end{array}$ & $\begin{array}{l}0.605^{* * * *} \\
(3.22)\end{array}$ & $\begin{array}{c}0.165 \\
(0.94)\end{array}$ & $\begin{array}{c}0.106 \\
(0.54)\end{array}$ & $\begin{array}{l}-0.026 \\
(-0.28)\end{array}$ & $\begin{array}{l}-0.187^{*} \\
(-1.77)\end{array}$ & $\begin{array}{l}-0.089 \\
(-0.78)\end{array}$ & $\begin{array}{l}-0.350^{* * *} \\
(-2.98)\end{array}$ & $\begin{array}{l}-0.322 \\
(-0.68)\end{array}$ & $\begin{array}{l}-0.406 \\
(-0.62)\end{array}$ & $\begin{array}{l}-0.756 \\
(-1.18)\end{array}$ & -0.10 \\
\hline Turkey & $\begin{array}{l}0.728^{* * *} \\
(2.62)\end{array}$ & $\begin{array}{l}0.787^{* *} \\
(2.56)\end{array}$ & $\begin{array}{l}0.633^{* *} \\
(2.06)\end{array}$ & $\begin{array}{c}0.103 \\
(0.43)\end{array}$ & $\begin{array}{l}-0.016 \\
(-0.10)\end{array}$ & $\begin{array}{l}-0.025 \\
(-0.16)\end{array}$ & $\begin{array}{l}-0.359 \\
(-1.58)\end{array}$ & $\begin{array}{l}-0.379^{*} \\
(-1.76)\end{array}$ & $\begin{array}{l}-0.758^{* * * *} \\
(-2.80)\end{array}$ & $\begin{array}{l}-1.458 \\
(-1.48)\end{array}$ & $\begin{array}{l}-2.406^{* * * *} \\
(-6.97)\end{array}$ & -0.12 \\
\hline \multicolumn{13}{|c|}{ Panel B: Frontier Markets } \\
\hline Dominican Republic & $\begin{array}{l}4.289 * * * \\
(3.54)\end{array}$ & $\begin{array}{l}2.673^{* * * *} \\
(2.78)\end{array}$ & $\begin{array}{r}0.400 \\
(0.13)\end{array}$ & $\begin{array}{c}0.115 \\
(0.16)\end{array}$ & $\begin{array}{l}0.202 \\
(1.04)\end{array}$ & $\begin{array}{l}0.128 \\
(1.20)\end{array}$ & $\begin{array}{l}0.334^{*} \\
(1.68)\end{array}$ & $\begin{array}{l}0.258 \\
(0.61)\end{array}$ & $\begin{array}{l}-0.260 \\
(-0.47)\end{array}$ & $\begin{array}{l}-1.053^{*} \\
(-1.66)\end{array}$ & $\begin{array}{l}-1.463^{* * *} \\
(-2.74)\end{array}$ & 0.22 \\
\hline Ecuador & $\begin{array}{l}3.631^{* * *} \\
(4.58)\end{array}$ & $\begin{array}{l}3.137^{* * *} \\
(5.89)\end{array}$ & $\begin{array}{r}1.631 \\
(1.59)\end{array}$ & $\begin{array}{c}0.474 \\
(0.28)\end{array}$ & $\begin{array}{l}-0.282 \\
(-1.18)\end{array}$ & $\begin{array}{l}-0.073 \\
(-0.67)\end{array}$ & $\begin{array}{l}-0.757 * * * \\
(-3.58)\end{array}$ & $\begin{array}{l}-1.704^{* * *} \\
(-4.95)\end{array}$ & $\begin{array}{l}-2.547^{* * *} \\
(-6.15)\end{array}$ & $\begin{array}{l}-3.812^{* * *} \\
(-5.55)\end{array}$ & $\begin{array}{l}-5.090^{* * *} \\
(-7.63)\end{array}$ & -0.38 \\
\hline Egypt & $\begin{array}{l}1.711^{* *} \\
(2.30)\end{array}$ & $\begin{array}{l}-0.204 \\
(-0.08)\end{array}$ & $\begin{array}{l}-0.144 \\
(-0.16)\end{array}$ & $\begin{array}{l}-0.122 \\
(-0.54)\end{array}$ & $\begin{array}{l}-0.255^{* *} \\
(-2.46)\end{array}$ & $\begin{array}{l}-0.144^{*} \\
(-1.71)\end{array}$ & $\begin{array}{l}-0.273^{*} \\
(-1.85)\end{array}$ & $\begin{array}{l}-0.194 \\
(-0.81)\end{array}$ & $\begin{array}{l}-0.156 \\
(-0.87)\end{array}$ & $\begin{array}{l}-0.137 \\
(-0.37)\end{array}$ & $\begin{array}{l}-0.004 \\
(-0.02)\end{array}$ & -0.17 \\
\hline Pakistan & $\begin{array}{r}0.269 \\
(0.36)\end{array}$ & $\begin{array}{c}0.609 \\
(0.86)\end{array}$ & $\begin{array}{l}0.550^{* * *} \\
(2.72)\end{array}$ & $\begin{array}{c}0.344 \\
(1.07)\end{array}$ & $\begin{array}{l}0.141 \\
(1.36)\end{array}$ & $\begin{array}{l}0.045 \\
(0.62)\end{array}$ & $\begin{array}{l}0.188 \\
(1.33)\end{array}$ & $\begin{array}{l}0.601 \\
(0.85)\end{array}$ & $\begin{array}{l}0.244 \\
(0.51)\end{array}$ & $\begin{array}{l}-0.149 \\
(-0.38)\end{array}$ & $\begin{array}{l}-0.098 \\
(-0.21)\end{array}$ & 0.21 \\
\hline
\end{tabular}

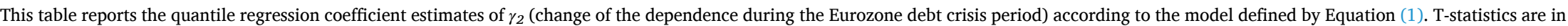
parentheses. The last column shows change in degree of dependence. 


\section{Appendix D. . Stock-bond correlations graphs}

See Fig. D1, Fig. D2, Fig. D3, Fig. D4.

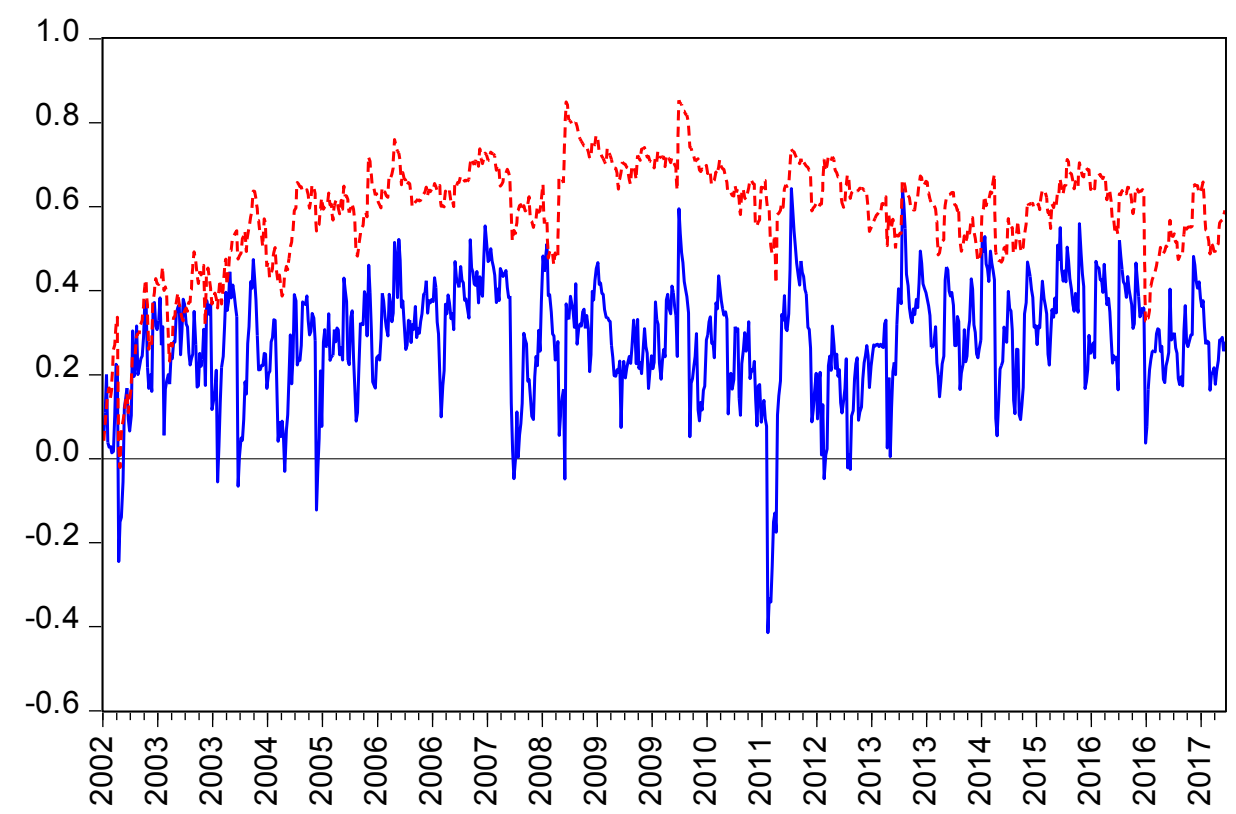

— GBI EM - Hedge ---- GBI EM - No Hedge

Fig. D1. Dynamic correlation estimates between global stock market returns and local currency emerging markets bond returns. Blue line shows correlations for the bond returns hedged to US dollar, while red line shows correlations for the bond returns without currency hedging. 


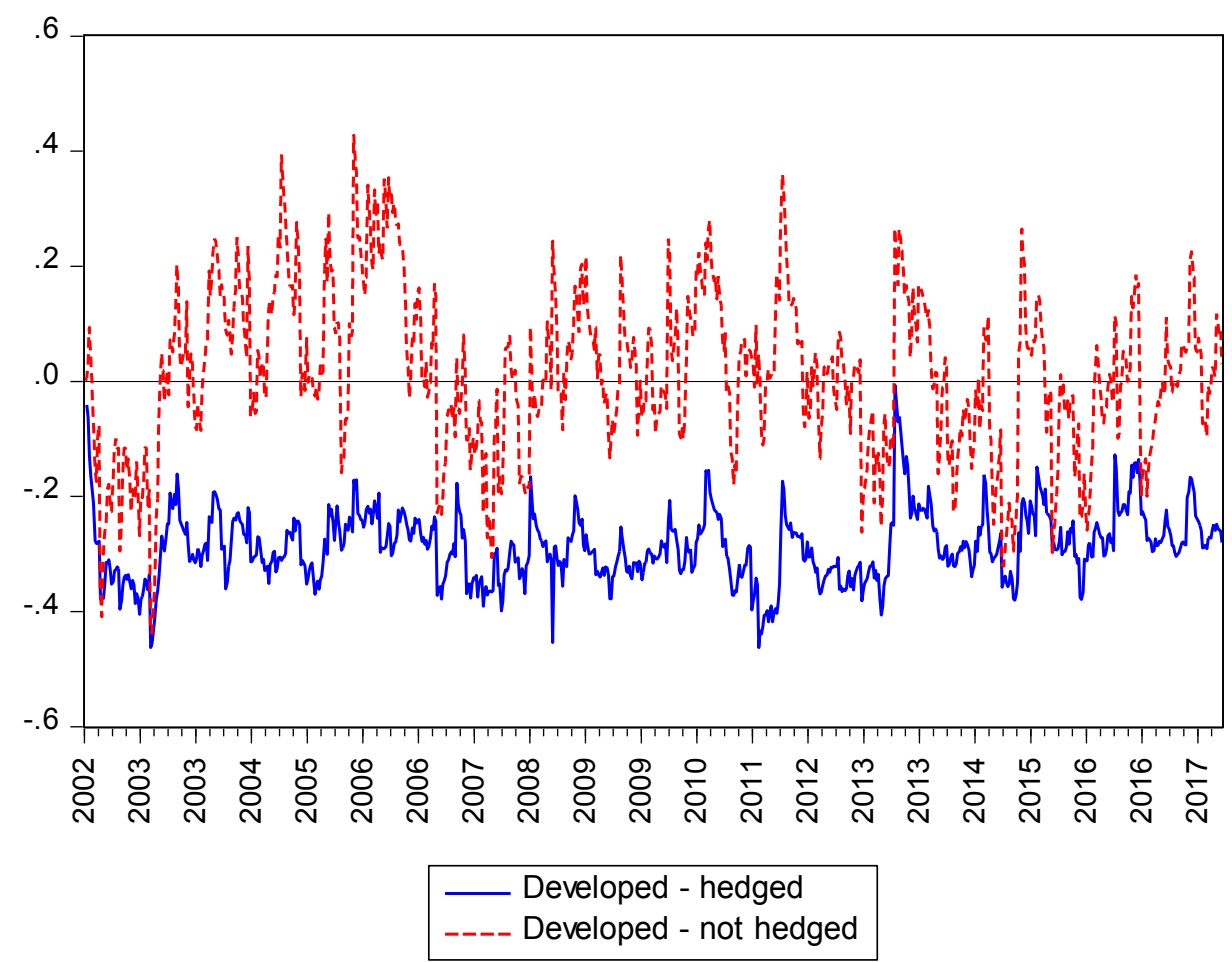

Fig. D2. Dynamic correlation estimates between global stock market returns and developed markets bond returns. Blue line shows correlations for the bond returns hedged to US dollar, while red line shows correlations for the bond returns without currency hedging. 


\section{$\mathrm{EMBI+}$}

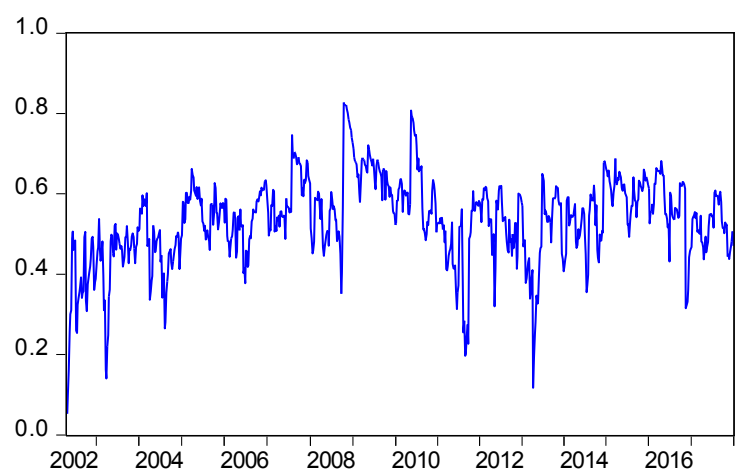

Asia
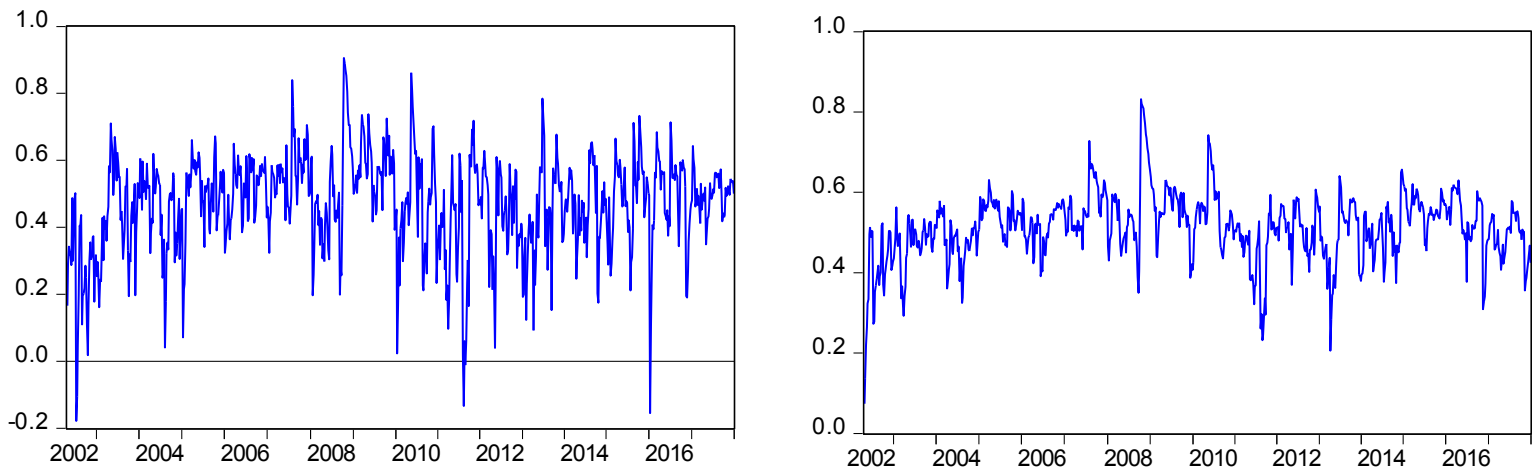

Europe

Africa
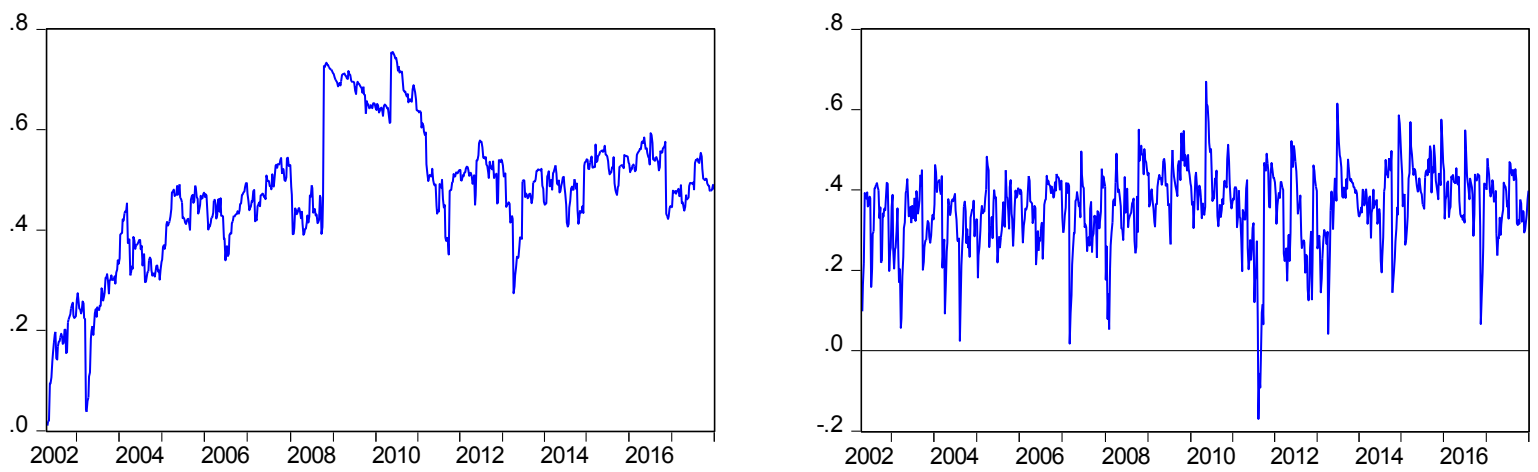

Fig. D3. Dynamic correlation estimates between global stock market returns and USD-denominated emerging markets bond returns on the aggregate level (EMBI + ) and regional level (Asia, Latin America, Europe, and Africa). 


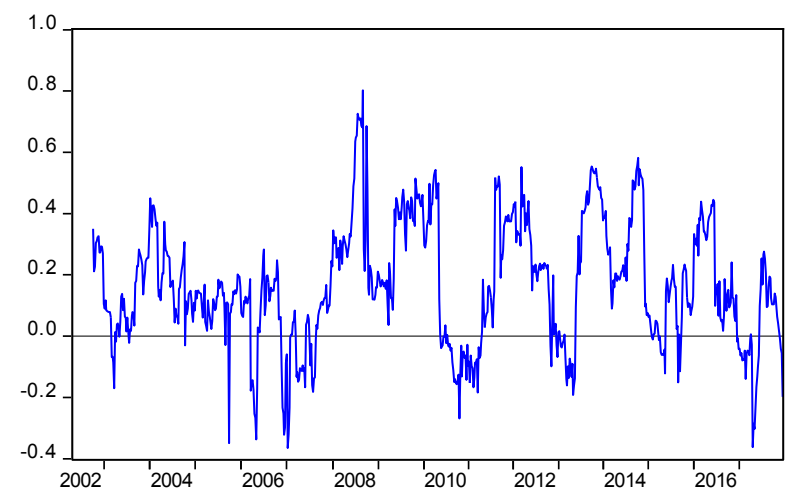

Frontier Africa

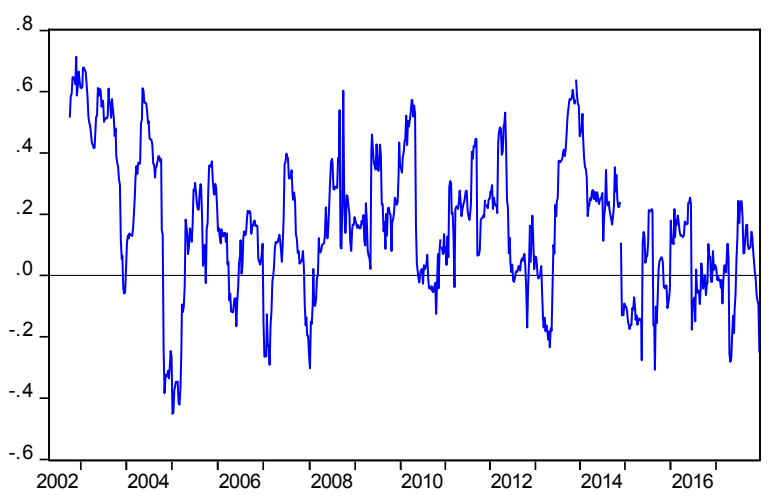

Frontier Latin America

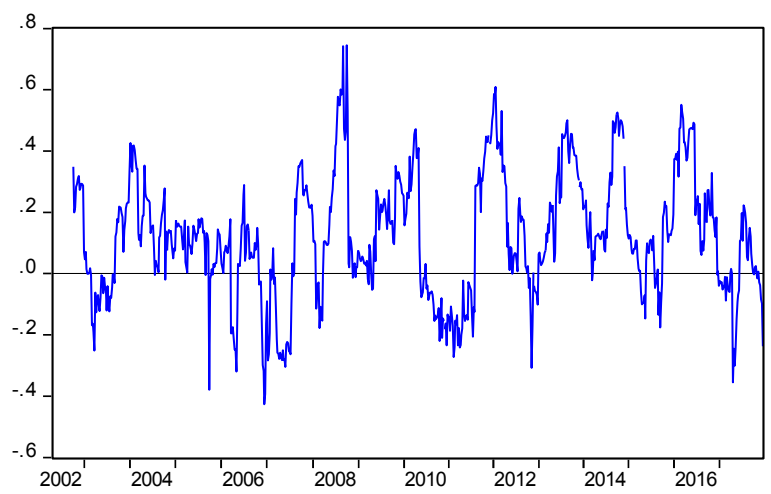

Fig. D4. Correlation estimates between global stock market returns and USD-denominated frontier markets bond returns on the aggregate level (NEXGEM) and regional level (Africa and Latin America). Middle East and Asian regions are not included in the analysis due to issue of data availability (larger time breaks in observations). Correlations are estimated by applying the rolling window correlation method instead of DCC model due to the convergence issue.

\section{Appendix E. Supplementary material}

Supplementary data to this article can be found online at https://doi.org/10.1016/j.intfin.2021.101385.

\section{References}

Abad, P., Chuliá, H., Gómez-Puig, M., 2014. Time-varying integration in European government bond markets. Eur. Financ. Manage. 20 (2), 270-290. https://doi.org/ 10.1111/j.1468-036X.2011.00633.x.

Abakah, E.J.A., Addo, E., Gil-Alana, L.A., Tiwari, A.K., 2021. Re-examination of international bond market dependence: evidence from a pair copula approach. Int. Rev. Financ. Anal. 74, 101678. https://doi.org/10.1016/j.irfa.2021.101678.

Adler, M., Dumas, B., 1980. The exposure of long-term foreign currency bonds. J. Financ. Quantit. Anal. 15 (4), 973-994. https://doi.org/10.2307/2330573.

Agur, I., Chan, M., Goswami, M., Sharma, S., 2019. On international integration of emerging sovereign bond markets. Emerg. Markets Rev. 38, 347-363. https://doi. org/10.1016/j.ememar.2018.11.006.

Alcock, J., Satchell, S., 2018. Asymmetric Dependence in Finance: Diversification, Correlation and Portfolio Management in Market Downturns. Wiley Finance Series. Amstad, M., Packer, F., Shek, J., 2020. Does sovereign risk in local and foreign currency differ? J. Int. Money Finance 101, 102099. https://doi.org/10.1016/j. jimonfin.2019.102099.

Ang, A., Bekaert, G., 2002. International asset allocation with regime shifts. Rev. Financ. Stud. 15 (4), 1137-1187. https://doi.org/10.1093/rfs/15.4.1137.

Ang, A., Chen, J., 2002. Asymmetric correlations of equity portfolios. J. Financ. Econ. 63 (3), 443-494. https://doi.org/10.1016/S0304-405X(02)00068-5.

Arellano, C., Ramanarayanan, A., 2012. Default and the maturity structure in sovereign bonds. J. Polit. Econ. 120 (2), 187-232. https://doi.org/10.1086/666589.

Aslanidis, N., Christiansen, C., 2014. Quantiles of the realized stock-bond correlation and links to the macroeconomy. J. Empiric. Financ. 28, 321-331. https://doi. org/10.1016/j.jempfin.2014.03.007.

Bae, K.-H., Karolyi, G.A., Stulz, R.M., 2003. A new approach to measuring financial contagion. Rev. Financ. Stud. 16 (3), 717-763. https://doi.org/10.1093/rfs/ hhg012.

Baele, L., Bekaert, G., Inghelbrecht, K., 2010. The determinants of stock and bond return comovements. Rev. Financ. Stud. 23 (6), 2374-2428. https://doi.org/ 10.1093/rfs/hhq014.

Baele, L., Bekaert, G., Inghelbrecht, K., Wei, M. (2020). Flights to safety. The Review of Financial Studies 33, 689-746. https://doi.org/10.1093/rfs/hhz055. 
Baur, D.G., Schulze, N., 2005. Coexceedances in financial markets - a quantile regression analysis of contagion. Emerg. Markets Rev. 6 (1), 21-43. https://doi.org/ 10.1016/j.ememar.2004.10.001.

Baur, D.G., Lucey, B.M., 2009. Flights and contagion - an empirical analysis of stock-bond correlations. J. Financ. Stability 5 (4), 339-352. https://doi.org/10.1016/j. jfs.2008.08.001.

Baur, D.G., Schulze, N., 2009. Financial market stability - a test. J. Int. Financ. Markets Institut. Money 19 (3), 506-519. https://doi.org/10.1016/j. intfin.2008.06.003.

Baur, D.G., 2013. The structure and degree of dependence: a quantile regression approach. J. Bank. Finance 37 (3), 786-798. https://doi.org/10.1016/j. jbankfin.2012.10.015.

Beine, M., Cosma, A., Vermeulen, R., 2010. The dark side of global integration: increasing tail dependence. J. Bank. Finance 34 (1), 184-192. https://doi.org/ 10.1016/j.jbankfin.2009.07.014.

Bekiros, S., Hammoudeh, S., Jammazi, R., Nguyen, D.K., 2018. Sovereign bond market dependencies and crisis transmission around the Eurozone debt crisis: a dynamic copula approach. Appl. Econ. 50 (47), 5031-5049. https://doi.org/10.1080/00036846.2018.1470313.

Bianconi, M., Yoshino, J.A., Machado de Sousa, M.O., 2013. BRIC and the U.S. financial crisis: an empirical investigation of stock and bond markets. Emerg. Markets Rev. 14, 76-109. https://doi.org/10.1016/j.ememar.2012.11.002.

Bhatta, B., Marshall, A., Thapa, C., 2017. Cost of sovereign debt and foreign bias in bond allocations. J. Int. Financ. Markets Instit. Money 51, 75-91. https://doi.org/ 10.1016/j.intfin.2017.09.001.

Bhatti, M.I., Nguyen, C.C., 2012. Diversification evidence from international equity markets using extreme values and stochastic copulas. J. Int. Financ. Markets Inst. Money 22 (3), 622-646. https://doi.org/10.1016/j.intfin.2012.02.004.

Bloomberg, 2012. Draghi Says ECB Will Do What's Needed to Preserve Euro: Economy. www.bloomberg.com/news/articles/2012-07-26/draghi-says-ecb-to-dowhatever-needed-as-yields-threaten-europe.

Boubaker, S., Nguyen, D.C., Piljak, V., Savvides, A., 2019. Financial development, government bond returns, and stability: International evidence. J. Int. Financ. Markets Inst. Money 61, 81-96. https://doi.org/10.1016/j.intfin.2019.02.006.

Brière, M., Chapelle, A., Szafarz, A., 2012. No contagion, only globalization and flight to quality. J. Int. Money Financ. 31 (6), 1729-1744. https://doi.org/10.1016/j. jimonfin.2012.03.010.

Broner, F., Lorenzoni, G., Schmukler, S., 2013. Why do emerging economies borrow short term? J. Eur. Econ. Assoc. 11, 67-100. https://doi.org/10.1111/j.15424774.2012.01094.x.

Bunda, I., Hamann, A.J., Lall, S., 2009. Correlations in emerging market bonds: the role of local and global factors. Emerg. Markets Rev. 10 (2), 67-96. https://doi. org/10.1016/j.ememar.2009.02.003.

Burger, J.D., Warnock, F.E., 2007. Foreign participation in local currency bond markets. Rev. Financ. Econ. 16 (3), 291-304. https://doi.org/10.1016/j. rfe.2006.09.001.

Burger, J.D., Warnock, F.E., Warnock, V.C., 2018. Currency matters: Analyzing international bond portfolios. J. Int. Econ. 114, 376-388. https://doi.org/10.1016/j. jinteco.2018.08.001.

Campbell, J.Y., Ammer, J., 1993. What moves the stock and bond markets? A variance decomposition for long-term asset returns. J. Financ. 48 (1), 3-37. https://doi. org/10.1111/j.1540-6261.1993.tb04700.x.

Caporin, M., Pelizzon, L., Ravazzolo, F., Rigobon, R., 2018. Measuring sovereign contagion in Europe. J. Financ. Stabil. 34, 150-181. https://doi.org/10.1016/j. jfs.2017.12.004.

Cappiello, L., Engle, R.F., Sheppard, K., 2006. Asymmetric dynamics in the correlations of global equity and bond returns. J. Financ. Econ. 4 (4), 537-572. https://doi. org/10.1093/jjfinec/nbl005.

Cappiello, L., Gérard, B., Kadareja, A., Simone Manganelli, S., 2014. Measuring comovements by regression quantiles. J. Financ. Econ. 12 (4), 645-678. https://doi. org/10.1093/jjifinec/nbu009.

Cayon, E., Thorp, S., Wu, E., 2018. Immunity and infection: emerging and developed market sovereign spreads over the global financial crisis. Emerg. Market. Rev. 34, 162-174. https://doi.org/10.1016/j.ememar.2017.11.006.

Chaieb, I., Errunza, V., Gibson Brandon, R., 2019. Measuring Sovereign Bond Market Integration. The Review of Financial Studies. https://doi.org/10.1093/rfs/ hhz107.

Chollete, L., Heinen, A., Valdesogo, A., 2009. Modeling international financial returns with a multivariate regime-switching copula. J. Financ. Economet. 7 (4), 437-480. https://doi.org/10.1093/jjfinec/nbp014.

Christiansen, C., 2014. Integration of European bond markets. J. Bank. Finance 42, 191-198. https://doi.org/10.1016/j.jbankfin.2014.01.022.

Christopher, R., Kim, S.-J., Wu, E., 2012. Do sovereign credit ratings influence regional stock and bond market interdependencies in emerging countries? J. Int. Financial Markets Inst. Money 22 (4), 1070-1089. https://doi.org/10.1016/j.intfin.2012.01.003.

Claeys, P., Vašícek, B., 2014. Measuring bilateral spillover and testing contagion on sovereign bond markets in Europe. J. Bank. Finance 46, 151-165. https://doi.org/ 10.1016/j.jbankfin.2014.05.011.

Connolly, R., Stivers, C., Sun, L., 2005. Stock market uncertainty and the stock-bond return relation. J. Financ. Quant. Anal. 40 (1), 161-194. https://doi.org/ $10.1017 /$ S0022109000001782.

d'Addona, S., Kind, A.H., 2006. International stock-bond correlations in a simple affine asset pricing model. J. Bank. Finance 30 (10), 2747-2765. https://doi.org/ 10.1016/j.jbankfin.2005.10.007.

De Goeij, P., Marquering, W., 2004. Modeling the conditional covariance between stock and bond returns: as multivariate GARCH approach. J. Financ. Econ. 2 (4), 531-564. https://doi.org/10.1093/jjfinec/nbh021.

Dimic, N., Kiviaho, J., Piljak, V., Äijö, J., 2016. Impact of financial market uncertainty and macroeconomic factors on stock-bond correlation in emerging markets. Res. Int. Business Finance 36, 41-51. https://doi.org/10.1016/j.ribaf.2015.09.001.

Dungey, M., Fry, R.A., Gonzalez-Hermosillo, B., Martin, V.L., 2006. Contagion in international bond markets during the Russian and LTCM crises. J. Financ. Stabil. 2, 1-27. https://doi.org/10.1016/j.jfs.2005.01.001.

Ehrmann, M., Fratzscher, M., 2017. Euro area government bonds - Fragmentation and contagion during the sovereign debt crisis. J. Int. Money Finance 70, 26-44. https://doi.org/10.1016/j.jimonfin.2016.08.005.

Eichengreen, B., Hausmann, R., Panizza, U., 2003. Currency mismatches, debt intolerance and original sin: why they are not the same and why it matters (No. w10036). National Bureau of Economic Research. https://doi.org/10.3386/w10036.

Embrechts, P., McNeil, A., Straumann, D., 2002. Correlation and dependence in risk management: properties and pitfalls. In: Dempster, M.A.H. (Ed.), Risk Management: Value at Risk and Beyond. Cambridge University Press, Cambridge.

Engle, R., 2002. Dynamic conditional correlation: a simple class of multivariate generalized autoregressive conditional heteroscedasticity models. J. Business Econ. Statist. 20 (3), 339-350. https://doi.org/10.1198/073500102288618487.

Federal Reserve Board of St, 2010. Louis. The Financial Crisis, A Timeline of Events and Policy Actions.

Farhi, E., Maggiori, M., 2018. A model of the international monetary system. Q. J. Econ. 133 (1), 295-355. https://doi.org/10.1093/qje/qjx031.

Filardo, A., George, J., Loretan, M., Ma, G., Munro, A., Shim, I., Wooldridge, P., Yetman, J., Zhu, H., 2010. The international financial crisis: timeline, impact and policy responses in Asia and the Pacific. BIS Papers 52, 21-82.

Forbes, K., Rigobon, R., 2002. No contagion, only interdependence: measuring stock market comovements. J. Finance 57 (5), 2223-2261. https://doi.org/10.1111/ 0022-1082.00494.

Garcia, R., Tsafack, G., 2011. Dependence structure and extreme comovements in international equity and bond markets. J. Bank. Finance 35 (8), 1954-1970. https:// doi.org/10.1016/j.jbankfin.2011.01.003.

Gennaioli, N., Martin, A., Rossi, S., 2018. Banks, government bonds, and default: What do the data say? J. Monetary Econ. 98, 98-113. https://doi.org/10.1016/j. jmoneco.2018.04.011. 
Gilchrist, Simon, Yue, Vivian, Zakrajšek, Egon, 2019. US monetary policy and international bond markets. Journal of Money, Credit and Banking 51 (S1), $127-161$. https://doi.org/10.1111/jmcb.12667.

Gómez-Puig, M., Sosvilla-Rivero, S., 2014. Causality and contagion in EMU sovereign debt markets. Int. Rev. Econ. Financ 33, 12-27. https://doi.org/10.1016/j. iref.2014.03.003.

Gulko, L., 2002. Decoupling. J. Portfolio Manage. 28 (3), 59-66. https://doi.org/10.3905/jpm.2002.319843.

Hartmann, P., Straetmans, S., Vries, C.D., 2004. Asset market linkages in crisis periods. Rev. Econ. Stat. 86 (1), 313-326. https://doi.org/10.1162/ 003465304323023831.

Hu, L., 2006. Dependence patterns across financial markets: a mixed copula approach. Appl. Financ. Econ. 16 (10), 717-729. https://doi.org/10.1080/ 09603100500426515.

Jeanneret, A., Souissi, S., 2016. Sovereign defaults by currency denomination. J. Int. Money Financ. 60, 197-222. https://doi.org/10.1016/j.jimonfin.2015.03.004.

Kim, S.-J., Lucey, B.M., Wu, E., 2006. Dynamics of bond market integration between established and accession European Union countries. J. Int. Financ. Markets Inst. Money 16 (1), 41-56. https://doi.org/10.1016/j.intfin.2004.12.004.

Koenker, R., Bassett, G., 1978. Regression quantiles. Econometrica: J. Econometric Society 46 (1), 33. https://doi.org/10.2307/1913643.

Koenker, R., Hallock, K.F., 2001. Quantile regression. J. Econ. Perspect. 15 (4), 143-156. https://doi.org/10.1257/jep.15.4.143.

Kumar, M.S., Okimoto, T., 2011. Dynamics of international integration of government securities' markets. J. Bank. Finance 35 (1), 142-154. https://doi.org/10.1016/ j.jbankfin.2010.07.019.

Lane, P.R., 2012. The European sovereign debt crisis. J. Econ. Perspect. 26 (3), 49-68. https://doi.org/10.1257/jep.26.3.49.

Li, X.-M., Zou, L.-P., 2008. How do policy and information shocks impact co-movements of China's T-bond and stock markets? J. Bank. Finance 32 (3), 347-359. https://doi.org/10.1016/j.jbankfin.2007.04.029.

Longin, F., Solnik, B., 2001. Extreme correlation of international equity markets. J. Financ. 56 (2), 649-676. https://doi.org/10.1111/0022-1082.00340.

Lucey, B.M., Vigne, S.A., Ballester, L., Barbopoulos, L., Brzeszczynski, J., Carchano, O., Dimic, N., Fernandez, V., Gogolin, F., González-Urteaga, A., Goodell, J.W., Helbing, P., Ichev, R., Kearney, F., Laing, E., Larkin, C.J., Lindblad, A., Lončarski, I., Ly, K.C., Marinč, M., McGee, R.J., McGroarty, F., Neville, C., O'HaganLuff, M., Piljak, V., Sevic, A., Sheng, X., Stafylas, D., Urquhart, A., Versteeg, R., Vu, A.N., Wolfe, S., Yarovaya, L., Zaghini, A., 2018. Future directions in international financial integration research - a crowdsourced perspective. Int. Rev. Financ. Anal. 55, 35-49. https://doi.org/10.1016/j.irfa.2017.10.008.

Lustig, H., Verdelhan, A., 2019. Does incomplete spanning in international financial markets help to explain exchange rates? Am. Econ. Rev. 109 (6), 2208-2244. https://doi.org/10.1257/aer.20160409.

Markwat, T., 2014. The rise of global stock market crash probabilities. Quantitat. Financ. 14 (4), 557-571. https://doi.org/10.1080/14697688.2013.848463.

Markwat, T., Kole, E., Van Dijk, D., 2009. Contagion as a domino effect in global stock markets. J. Bank. Finance 33 (11), 1996-2012. https://doi.org/10.1016/j. jbankfin.2009.05.008.

Ning, C., 2010. Dependence structure between the equity market and the foreign exchange market - a copula approach. J. Int. Money Financ. 29 (5), 743-759. https://doi.org/10.1016/j.jimonfin.2009.12.002.

Okimoto, T., 2008. New evidence on asymmetric dependence structures in international equity markets. J. Financ. Quantitat. Anal. 43 (3), 787-815. https://doi.org/ 10.1017/S0022109000004294.

Okimoto, T., 2014. Asymmetric increasing trends in dependence in international equity markets. J. Bank. Finance 46, 219-232. https://doi.org/10.1016/j. jbankfin.2014.05.025.

Ottonello, P., Perez, D.J., 2019. The currency composition of sovereign debt. Am. Econ. J.: Macroecon. 11 (3), 174-208. https://doi.org/10.1257/mac.20180019.

Panchenko, V., Wu, E., 2009. Time-varying market integration and stock and bond return concordance in emerging markets. J. Bank. Finance 33 (6), 1014-1021. https://doi.org/10.1016/j.jbankfin.2008.10.016.

Patton, A.J., 2004. On the out-of-sample importance of skewness and asymmetric dependence for asset allocation. J. Financ. Economet. 2 (1), 130-168. https://doi. org/10.1093/jjifinec/nbh006.

Patton, A.J., 2006. Modelling asymmetric exchange rate dependence. Int. Econ. Rev. 47 (2), 527-556. https://doi.org/10.1111/iere.2006.47.issue-210.1111/j.14682354.2006.00387.x.

Patton, A.J., 2012. A review of copula models for economic time series. J. Multivariate Anal. 110, 4-18. https://doi.org/10.1016/j.jmva.2012.02.021.

Piljak, V., 2013. Bond markets co-movement dynamics and macroeconomic factors: Evidence from emerging and frontier markets. Emerg. Market. Rev. 17, 29-43. https://doi.org/10.1016/j.ememar.2013.08.001.

Piljak, V., Swinkels, L., 2017a. Frontier and emerging government bond markets. Emerg. Market. Rev. 30, 232-255. https://doi.org/10.1016/j.ememar.2015.10.002.

Piljak, V., Swinkels, L., 2017b. Fundamental indexation for developed, emerging, and frontier government bond markets. J. Asset Manage. 18 (5), 405-420. https:// doi.org/10.1057/s41260-017-0045-8.

Pires, P., Pereira, J.P., Martins, L.F., 2015. The empirical determinants of credit default swap spreads: a quantile regression approach. Eur. Financ. Manage. 21 (3), 556-589. https://doi.org/10.1111/eufm.v21.310.1111/j.1468-036X.2013.12029.x.

Rodriguez, J.C., 2007. Measuring financial contagion: a copula approach. J. Empiric. Financ. 14 (3), 401-423. https://doi.org/10.1016/j.jempfin.2006.07.002.

Samarakoon, L.P., 2017. Contagion of the eurozone debt crisis. J. Int. Financ. Markets Institut. Money 49, 115-128. https://doi.org/10.1016/j.intfin.2017.03.001.

Šimović, P.P., Tkalec, M., Vizek, M., Lee, J., 2016. Time-varying integration of the sovereign bond markets in European post-transition economies. J. Empirical Finance 36, 30-40. https://doi.org/10.1016/j.jempfin.2015.12.005.

Yang, J., Zhou, Y., Wang, Z., 2009. The stock-bond correlation and macroeconomic conditions: One and a half centuries of evidence. J. Bank. Finance 33 (4), 670-680. https://doi.org/10.1016/j.jbankfin.2008.11.010.

Yuan, K., 2005. Asymmetric price movements and borrowing constraints: a rational expectations equilibrium model of crises, contagion, and confusion. J. Finance 60, 379-411. https://doi.org/10.1111/j.1540-6261.2005.00733.x. 